Portland State University

PDXScholar

$5-24-2021$

\title{
Method of Modeling the Swing Equation Using Time Synchronized Measurements
}

Robert Matthew Ferraro

Portland State University

Follow this and additional works at: https://pdxscholar.library.pdx.edu/open_access_etds

Part of the Power and Energy Commons

Let us know how access to this document benefits you.

\section{Recommended Citation}

Ferraro, Robert Matthew, "Method of Modeling the Swing Equation Using Time Synchronized Measurements" (2021). Dissertations and Theses. Paper 5729.

https://doi.org/10.15760/etd.7600

This Thesis is brought to you for free and open access. It has been accepted for inclusion in Dissertations and Theses by an authorized administrator of PDXScholar. Please contact us if we can make this document more accessible: pdxscholar@pdx.edu. 
Method of Modeling the Swing Equation Using Time Synchronized Measurements

by

Robert Matthew Ferraro

A thesis submitted in partial fulfillment of the requirements for the degree of

\author{
Master of Science \\ in \\ Electrical and Computer Engineering
}

Thesis Committee:

Robert Bass, Chair

Mahima Gupta

John M. Acken

Portland State University

2021 
(C) 2021 Robert Matthew Ferraro 


Abstract

In three phase, high-voltage transmission systems, synchronous generators accelerate or decelerate to adapt to changing power transfer requirements that occur during system disturbances. In network electrical power systems, frequency changes constantly based on system dynamics. Modeling network dynamics from oscillations and transients using time-synchronized measurements can provide real-time information, including angular displacements, voltage and current phasors, frequency changes, and rate of signal system decay from positive-sequence components.

Power system voltage and current waveforms are not steady-state sinusoids, especially during system disturbances. These waveforms contain sustained harmonic and non-harmonic components. Additionally, because of faults and other switching electromagnetic transients, there may be step changes in the magnitude and phase angles of these waveforms. Resonances in the power network create additional frequencies. Other disturbances may exhibit relatively slow changes in phase angles and magnitudes due to oscillations of machine rotors during electromechanical disturbances.

Power System stability is that property of a system that enables the synchronous machines of the system to respond to a disturbance and return to normal operating conditions. To determine the system characteristics, analysis of system stability can be performed by transient, dynamic and steady-state stability studies. 
Power systems are heavily inter-connected with many hundreds of machines that interact dynamically through the medium of their high voltage networks. Transient stability studies are performed to study the power system electromechanical dynamic behavior, and are aimed to determine if the system will remain in synchronism following major disturbances. The measurement equipment and computer modeling required, both in time and cost, can be extensive.

The equation governing the motion of the rotor of a synchronous machine is based on an elementary principle of dynamics, where accelerating torque is the product of the moment of inertia of the rotor times its angular acceleration. During a disturbance, how the rotor will accelerate or decelerate is described in relative motion by the swing equation.

This research uses archived Phasor Measurement Unit (PMU) data obtained from the Bonneville Power Administration (BPA) to demonstrate a feasible technique for transient stability system analysis. This work demonstrates a practical method of using Rate of Change of Frequency (ROCOF) from PMU data with a MATLAB analysis fit program to determine the system coefficients used to calculate the damping coefficient $D$, and inertia constant $H$, which are necessary to create a practical swing equation.

Because PMU data have become an important component in wide-area measurements used in many power systems, PMU data are readily available to make quick, useful approximations. With the event of a large disturbance that excites system dynamics, valuable data are obtained from PMUs with useful coefficients around the power system.

The method described in this work evaluates PMU data with a MATLAB fit program, 
which successfully analyzes ROCOF measurements under transient conditions through signal decay to provide quality measurements and determine the coefficients of the swing equation. 
Dedication

Dedication to My Parents 


\section{Acknowledgements}

Special thanks to Tylor Slay, your work made this effort possible, and to Anthony Faris at BPA for his contributions. I would like to gratefully acknowledge my adviser and friend, Dr. Robert Bass, for his intellectual insight, guidance and support; as well as thesis committee

members Mahima Gupta and John M. Acken. It has been an honor to have the opportunity to work with such insightful and talented minds. 


\section{Contents}

$\begin{array}{ll}\text { Abstract } & \text { i }\end{array}$

Dedication iv

$\begin{array}{ll}\text { Acknowledgements } & \text { v }\end{array}$

List of Tables $\quad$ viii

List of Figures $\quad$ ix

Acronyms $\quad$ xi

1 Introduction $\quad 1$

1.1 Problem Statement . . . . . . . . . . . . . . . . . . 1

1.2 Objectives of Work . . . . . . . . . . . . . . 4

2 Design Methodology 5

2.1 Why D \& H ..................... 5

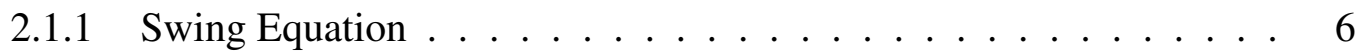

2.1.2 The Power-Angle . . . . . . . . . . . . . . . . . . 14

2.1.3 Synchronizing Power Coefficients . . . . . . . . . . . . . . . 19

2.1.4 Equal-Area Criterion . . . . . . . . . . . . . . . . . 22

2.1.5 Why are there stability studies ............. 30

2.1.6 Small-Signal Stability . . . . . . . . . . . . . . . . . . 34

2.1.7 Transient Stability . . . . . . . . . . . . . . . . . . . . . . 42

2.1.8 Damped Oscillations . . . . . . . . . . . . . . . . 43

2.1.9 Characteristic Equations . . . . . . . . . . . . . . . . . . . . 48

2.2 Numerical Solutions of the Swing Equation . . . . . . . . . . . . 51

2.2.1 Step-by-Step Method . . . . . . . . . . . . . . . . . 52

2.2 .2 Euler Method . . . . . . . . . . . . . . . . . . . . 57

2.2.3 Modified Euler Method . . . . . . . . . . . . . . . . . . . . 59

2.2.4 Runge-Kutta (R-K) Methods . . . . . . . . . . . . . . . . . . . 60

2.2.5 Second-Order (R-K) Method . . . . . . . . . . . . . . . . 60

2.2.6 Fourth-Order (R-K) Method . . . . . . . . . . . . . . . . . . 60

2.3 How do D \& H characterize the system . . . . . . . . . . . . . 61 
2.4 How, historically, have D \& $\mathrm{H}$ been determined . . . . . . . . . . . . . . . 64

2.5 PMU background . . . . . . . . . . . . . . . . . 73

3 Results \& Analysis $\quad 78$

3.1 How to derive D \& $\mathrm{H}$ from PMU data . . . . . . . . . . . . . . . 78

3.2 Fit Filter Program . . . . . . . . . . . . . . . . . . . . 79

3.3 PMU Test Data . . . . . . . . . . . . . . . . . 81

3.4 Sample preparation prior to using fit filter program . . . . . . . . 83

3.5 Signal Outliers . . . . . . . . . . . . . . . . . 85

3.6 Plots . . . . . . . . . . . . . . . . . 87

4 Discussion $\quad 92$

4.1 Potential Sources of Error . . . . . . . . . . . . . . . . . . . . . . . . . . . . 92

4.2 Improvements . . . . . . . . . . . . . . . 93

4.3 BPA Chief Jo Example . . . . . . . . . . . . . . . . . . . . . . . . . . . . . . . . . .

4.4 Discussion of Results . . . . . . . . . . . . . . . . 95

5 Conclusion $\quad 100$

$\begin{array}{ll}\text { Bibliography } & 104\end{array}$

Appendix A: X-Y Plots 109

A.1 X-Y Plots . . . . . . . . . . . . . . . . . . 109

Appendix B: Event Signals $\quad 116$

B.1 Event Signals . . . . . . . . . . . . . . . . . . 116

Appendix C: Fit Filter Program 130

C.1 MATLAB Code . . . . . . . . . . . . . . . . . 130 


\section{List of Tables}

2.1 Suggested Form for Step-by-Step Angle-Time Calculations . . . . . . . . . . 73

3.1 Statistical Comparison Event 3 PMU Data: All vs. Truncated . . . . . . . . . 85

3.2 Statistical Comparison All PMU Data vs. Truncated PMU Data . . . . . . . . . 91

4.1 Statistical Comparison Damping Coefficient vs Inertia Constant _ . . . . . . . 97

4.2 Comparison of 12 PMU outliers: Damping Coefficient vs Inertia Constant . . . 98

4.327 PMUs Coefficients and Constants . . . . . . . . . . . . . . . . . . 99

5.1 Typical Good Values of Coefficients and Constants . . . . . . . . . . . 102 


\section{List of Figures}

2.1 Large, networked power system as a single generator. . . . . . . . . . . . 11

2.2 Generator Circuit and Phasor Diagram . . . . . . . . . . . . . . . . . . 15

2.3 Schematic Diagram Transient Reactances . . . . . . . . . . . . . 16

2.4 Power-Angle Equation Plot . . . . . . . . . . . . . . . . . . 20

2.5 SMIB System with Short Transmission line . . . . . . . . . . . . . . 23

2.6 Power-Angle Curves . . . . . . . . . . . . . . . . . 25

2.7 Power-Angle Curves with critical-clearing . . . . . . . . . . . . . . . . . 29

2.8 Nature of small-disturbance response . . . . . . . . . . . . . . . . . . . . . . . . . . . . . . . . . . .

2.9 Eigenvalues damped cosine oscillations in complex plane . . . . . . . . . . . 41

2.10 Damped Cosine Oscillation . . . . . . . . . . . . . . . . . . 46

2.11 Actual and assumed values of $P_{a}, \omega_{r}$, and $\delta$ as functions of time . . . . . . 54

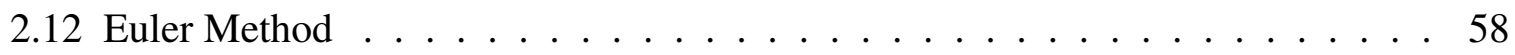

2.13 Inertia Constants from $1937 \ldots \ldots \ldots$. . . . . . . . . . 70

2.14 Synchrophasor illustration using the UTC reference . . . . . . . . . . . . 75

2.15 Synchrophasors associate phasor measurements to an absolute time reference. . 76

2.16 Voltage signal angles at different locations . . . . . . . . . . 76

3.1 Flow diagram for the fit filter program . . . . . . . . . . . . . . 82

3.2 PMU data sample preparation for fit filter program . . . . . . . . . . . . . 86

3.3 Example of a PMU data outlier. . . . . . . . . . . . . . . . . . . . 88

3.4 Statistical box-and-whisker plots of all PMU data. . . . . . . . . . . . . . . 90

A.1 PMU Data $\zeta \ldots \ldots \ldots \ldots \ldots \ldots \ldots$

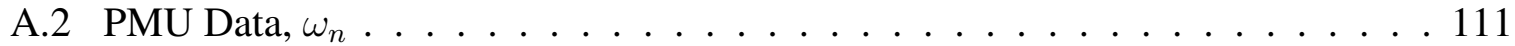

A.3 PMU Data, GOF . . . . . . . . . . . . . . . . . . . . . . . . . . . . . . . . . . . .

A.4 PMU Data, $\tau \ldots \ldots \ldots \ldots \ldots \ldots \ldots \ldots$

A.5 PMU Data, $\frac{D}{P_{s}} \ldots \ldots \ldots \ldots \ldots \ldots \ldots \ldots \ldots \ldots \ldots$

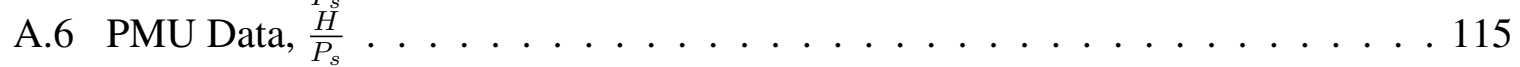

B.1 Event 1, Outage on DC Intertie . . . . . . . . . . . . . 117

B.2 Event 2, Line trip . . . . . . . . . . . . . . . . . . . 118

B.3 Event 3, Insertion of Chief Joseph brake . . . . . . . . . . . . . . . . 119

B.4 Event 4, Generator outage . . . . . . . . . . . . . . . . . 120

B.5 Event 5, Generator outage . . . . . . . . . . . . . . . . 121

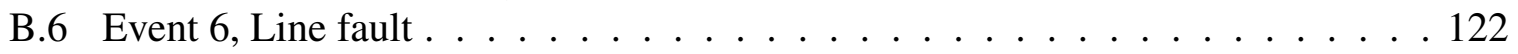

B.7 Event 7 , Line fault . . . . . . . . . . . . . . . . . 123 
B.8 Event 8, Line fault . . . . . . . . . . . . . . . . . . . . . . . . . . 124

B.9 Event 9, Outage on DC intertie . . . . . . . . . . . . . . . . 125

B.10 Event 10, Line fault . . . . . . . . . . . . . . . . . 126

B.11 Event 11, Insertion of Chief Joseph brake . . . . . . . . . . . . . . 127

B.12 Event 12, Insertion of Chief Joseph brake . . . . . . . . . . . . . . 128

B.13 Event 13, Insertion of Chief Joseph brake . . . . . . . . . . . . . . . 129

C.1 Fit program code . . . . . . . . . . . . . . . . . . . . . . 131

C.2 Fit program code . . . . . . . . . . . . . . . . . . 132

C.3 Fit program code . . . . . . . . . . . . . . . . . . 133 


\section{Acronyms}

GOF Goodness of Fit

BPA Bonneville Power Administration

DC Direct Current

GPS Global Positioning System

IRIG-B Inter-range Instrumentation Group Time Code Format B

PMU Phasor Measurement Unit

ROCOF Rate of Change of Frequency

SCDR Symmetrical Component Distance Relay

SMIB Single Machine Infinite Bus

UTC Coordinated Universal Time 


\section{Introduction}

\subsection{Problem Statement}

Stability is the ability of a power system to remain in synchronous equilibrium under steady operating conditions, and to regain a state of equilibrium after a disturbance has occurred [1]. Since stability is a problem associated with the parallel operation of synchronous machines, it might be suspected that the problem appeared when synchronous machines were first operated in parallel. The first serious problem of parallel operation, however, was not stability, but hunting [2]. Hunting are sustained oscillations in speed due to the periodic variations in torque applied to generators. Prior to 1890 parallel operation of synchronous machines was accomplished in isolated instances [3]. The problem did not assume importance until after the change from belted machines to engine driven machines, and from smooth to slotted armature construction.

Most of the ac generators were driven by direct-connected steam engines. The pulsating torque by those engines gave rise to hunting, which was sometimes aggravated by resonance between the period of pulsation of prime mover torque and the electromechanical period of the power system [2].

The periodic variations in the torque applied to the generators caused periodic variations in speed. The resulting periodic variations in voltage and frequency were transmitted to the 
motors connected to the system. Oscillations of the motors caused by these variations in voltage and frequency would sometimes cause motors to lose synchronism entirely if their natural frequency of oscillation coincided with the frequency of oscillation caused by the engines driving the generators [4].

To mitigate the oscillations, damper windings were first developed to reduce seriousness of hunting, and later the problem largely disappeared with the use of turbines. But with the development of large, heavily interconnected systems with hundreds of machines connected by long transmission lines, the stability of power systems has become more complex. In three phase, high-voltage transmission systems, synchronous generators accelerate or decelerate to adapt to changing power transfer requirements that occur during system disturbances. In actual network systems, voltage and frequency changes constantly, based on system dynamics [4].

The stability problem is concerned with the behavior of synchronous machines after they have been perturbed, causing a readjustment of the voltage angles of the synchronous machines. If this perturbation does not involve any net change in power, the machines should return to their original state. If an imbalance between the supply and demand is created by a change in load, in generation, or in network conditions, a new operating state is necessary [5].

Adjustment to the new operating condition is called the transient period. The system behavior during this time is the dynamic system performance, which is of concern in defining system stability [5]. The main criteria for stability is that the synchronous machine maintain 
synchronism at the end of the transient period. The transient following a system perturbation is oscillatory in nature. If the system is stable, these oscillations will be damped to a new stable operating condition. Power system stability requires that system oscillations be damped. This damped condition is referred to as asymptotic stability, where the system contains inherent forces that tend to reduce oscillations [5].

Stability studies, which evaluate the impact of disturbances on the electromechanical dynamic behavior of a power system, are either transient or steady-state. System models used in such studies are complex. Disturbances can be large or small depending on the origin. Transmission faults, sudden load changes, loss of generation, and line switching are examples where nonlinear equations describing the dynamics of a power system cannot be linearized for purpose of analysis.

The ability of a generator or groups of generators to remain in synchronism immediately following a sudden disturbance is the initial swing. However, following a large disturbance a transiently-stable condition is obtained. Transient stability is typically viewed as first swing stability. Signal analysis can be performed during this first-swing following a system fault to obtain useful data. By analyzing the ROCOF from PMU data, in the response of a second-order system of signal decay, the ROCOF can be linearized and filtered to obtain coefficients of the swing equation. This research characterizes a practical method for determining coefficients of the swing equation as a tool for system characterization. 


\subsection{Objectives of Work}

A literature study was performed to assess the applications in small signal analysis and the impact of this project. It is apparent that time-synchronized phasor measurements have become an important component of wide area measurements in powers systems, and the custom applications are extensive. The goal of this study was to develop a useful method to determine the coefficients of the swing equation with PMU data using a unique MATLAB analysis program. The simulations and results were then used in the development of this method. The research here distinguishes itself for being a practical method to provide a quick check when evaluating a system's swing equation. By analyzing positive-sequence frequency data from three different PMU locations at multiple transmission-level events, signal data can be used to determine useful swing equation coefficients.

From this work, a method to optimize starting data sets are discussed. The purpose of this methodology review was to mitigate ancillary transients and improve the program's filtered best-fit output. Additionally, an automatic function that could be incorporated into the filter program is discussed, but was not used for this study. 


\section{Design Methodology}

\subsection{Why D \& H}

The modern view of the stability problem dates from the 1924 Winter Convention of the American Institute of Electrical Engineers when a group of papers [3] called attention to the importance of the problem and presented the results of the first laboratory tests on miniature systems proportioned to simulate a power system having a long transmission line. Another important step was taken 1925 when the first field tests [6] [7] on stability were made on the system of Pacific Gas and Electric Company. Much additional practical information [8] on the problem was obtained by transient recording apparatus, first installed on the system of the Southern California Edison Company. Stability, in the sense employed by the First Report of Power System Stability [9], is concerned with the successful parallel operation of ac machines as affected by the magnitude of power transmitted. This problem existed since the beginning of parallel operation. During a period from 1924 to 1933, the theory of system stability was carefully investigated.

To better understand the Damping Coefficient (D) and the Inertia Constant $(\mathrm{H})$, we first look at the Swing Equation. 


\subsubsection{Swing Equation}

If you consider a synchronous generator with electromagnetic torque $T_{e}$ running at synchronous speed $\omega_{s m}$, during normal steady-state operation, the mechanical torque $T_{m}=T_{e}$. If there is a perturbation in the system it will result in an acceleration or deceleration torque, where $T_{a}=T_{m}-T_{e}$. With $T_{a}>0$ if accelerating, and $T_{a}<0$ if decelerating.

By the law of rotation, the swing equation governs the motion of the machine rotor relating the inertia torque to the resultant of the mechanical and electrical torques of the rotor [5]. Both Anderson and Kimbark provide an excellent discussion of units and dimensional analysis of the swing equation [2][5].

$$
J \frac{d^{2} \theta_{m}}{d t^{2}}=T_{a}=T_{m}-T_{e} \quad(N \cdot m)
$$

Where the symbols have the following definitions:

- $J=$ the total moment of inertia of the rotor masses, in $\mathrm{kg} \cdot \mathrm{m}^{2}$

- $\theta_{m}=$ the angular displacement of the rotor with respect to a stationary axis, in mechanical radians

- $t=$ time in seconds

- $T_{m}=$ the mechanical or shaft torque supplied by the prime mover less retarding torque to rotational losses, $N \cdot m$

- $T_{e}=$ the net electrical or electromagnetic torque, in $N \cdot m$ 
In equation $2.1, J$ is the total moment of inertia of the rotating masses. $\theta_{m}$ is the rotor angular position with respect to a stationary axis.

$$
\theta_{m}=\omega_{s m} t+\delta_{m}
$$

Equation 2.2, is the angular position, where $\omega_{s m}$ is the synchronous speed of the machine, and $\delta_{m}$ is the angular displacement of the rotor. Since $\theta_{m}$ is measured with respect to a stationary axis, it is an absolute measure of the rotor angle. Taking the derivatives of equation 2.1 with respect to time provide equations 2.3 and 2.4.

$$
\begin{gathered}
\frac{d \theta_{m}}{d t}=\omega_{s m}+\frac{d \delta_{m}}{d t} \\
\frac{d^{2} \theta_{m}}{d t^{2}}=\frac{d^{2} \delta_{m}}{d t^{2}}
\end{gathered}
$$

Equation 2.3 shows that the rotor angular velocity $d \theta_{m} / d t$ is constant and equals synchronous speed only when $d \delta_{m} / d t$ is zero. Therefore, $d \delta_{m} / d t$ represents the deviation of the rotor speed from synchronism. Substituting equation 2.4 in equation 2.1 , we obtain

$$
J \frac{d^{2} \delta_{m}}{d t^{2}}=T_{a}=T_{m}-T_{e} \quad(N \cdot m)
$$

Now it is convenient to show the angular velocity of the rotor in equation 2.6.

$$
\omega_{m}=\frac{d \theta_{m}}{d t}
$$

Power equals torque times angular velocity. Multiply equation 2.5 by $\omega_{m}$ to obtain the power equation 2.7 . 


$$
J \omega_{m} \frac{d^{2} \delta_{m}}{d t^{2}}=P_{a}=P_{m}-P_{e}
$$

Where $P_{m}$ is the shaft power input to the machine less rotational losses. $P_{e}$ is the electrical power crossing its air gap, and $P_{a}$ is accelerating power which accounts for any unbalance between $P_{m}$ and $P_{e}$.

The coefficient $J \omega_{m}$ is the angular momentum of the rotor; at synchronous speed $\omega_{s m}$, it is denoted by $M$ and called the inertia constant of the machine.

$$
M \frac{d^{2} \delta_{m}}{d t^{2}}=P_{a}=P_{m}-P_{e}
$$

It is noted that the angular momentum $M$ is not strictly constant [2] because the angular velocity $\omega_{m}$ varies somewhat during the swings which follow a disturbance. However, in practice, $\omega_{m}$ does not differ significantly from synchronous speed when the machine is stable, and since power is more convenient in calculations than torque, equation 2.8 is preferred.

In machine data developed for stability studies, another constant related to inertia is the $H$ constant, which is defined by equation 2.9 .

$$
\begin{gathered}
\mathrm{H}=\frac{\text { stored kinetic energy in megajoules at synchronous speed }}{\text { machine rating in } M V A} \\
H=\frac{\frac{1}{2} J \omega_{s m}^{2}}{S_{\text {mach }}}=\frac{\frac{1}{2} M \omega_{\text {sm }}}{S_{\text {mach }}} \quad(M J / M V A)
\end{gathered}
$$


Where $S_{\text {mach }}$ is the three-phase rating of the machine in megavoltamperes. Solving for $M$ we obtain equation 2.10 in mechanical radians.

$$
M=\frac{2 H}{\omega_{s m}} S_{\text {mach }} \quad\left(M J / \operatorname{rad}_{m}\right)
$$

When we substitute $M$ into equation 2.10, we obtain equation 2.11. Where $\delta_{m}$ is expressed in mechanical radians and $\omega_{s m}$ is expressed in mechanical radians per second.

$$
\frac{2 H}{\omega_{s m}} \frac{d^{2} \delta_{m}}{d t^{2}}=\frac{P_{a}}{S_{\text {mach }}}=\frac{P_{m}-P_{e}}{S_{\text {mach }}} \quad \text { (unit less) }
$$

Thus, we can write the equation in per unit form where both $\delta$ and $\omega_{s}$ have consistent units, which may be mechanical or electrical degrees or radians. $\mathrm{H}$ and $\mathrm{t}$ have consistent units since megajoules per megavoltamperes is in units of time in seconds and $P_{a}, P_{m}$, and $P_{e}$ must be in same base as $\mathrm{H}$. When subscript $m$ is associated with $\omega, \omega_{s}$, and $\delta$, it means mechanical units are being used; otherwise, electrical units are implied.

$$
\begin{gathered}
\frac{2 H}{\omega_{s}} \frac{d^{2} \delta}{d t^{2}}=P_{a}=P_{m}-P_{e} \quad(\text { per unit) } \\
\text { where } M=\frac{2 H}{\omega_{s}}
\end{gathered}
$$

Equation 2.12 is the swing equation of the machine, and is the fundamental equation which governs the rotational dynamics of the synchronous machine in stability studies.

Additionally, $\omega_{s}$ is the synchronous speed in electrical units. The swing equation with an electrical frequency of $f$, Hertz becomes equation 2.3.

$$
\left.\frac{H}{\pi f} \frac{d^{2} \delta_{m}}{d t^{2}}=P_{a}=P_{m}-P_{e} \quad \text { (per unit }\right)
$$


Equation 2.3 applies when $\delta$ is in electrical radians.

$$
\left.\frac{H}{180 f} \frac{d^{2} \delta_{m}}{d t^{2}}=P_{a}=P_{m}-P_{e} \quad \text { (per unit }\right)
$$

Equation 2.15 applies when $\delta$ is in electrical degrees. The swing equation 2.12 is the fundamental equation which governs the rotational dynamics of a synchronous machine in stability studies. It is a second order differential equation, which can be approximated as two first order differential equations 2.16 and 2.17.

$$
\begin{gathered}
\frac{2 H}{\omega_{s}} \frac{d \omega}{d t}=P_{m}-P_{e} \quad(\text { per unit }) \\
\frac{d \delta}{d t}=\omega-\omega_{s}
\end{gathered}
$$

In a stability study for a large system with many machines geographically dispersed over a wide area, it is desirable to minimize the number of swing equations to be solved. This can be done if the transmission line fault, or other disturbance on the system, affects the machines within a network so that their rotors swing together. In such cases, the machines within the network can be combined into a single equivalent machine just as if their rotors were mechanically coupled and only one swing equation needs to be created for an equivalent "generator" representing the network, as illustrated in Figure 2.1.

If we look at two generators in a single plant that are connected to the same bus and are electrically remote from a network disturbance, the swing equations on the common base are equations 2.18 and 2.19 . 
Measurement Point

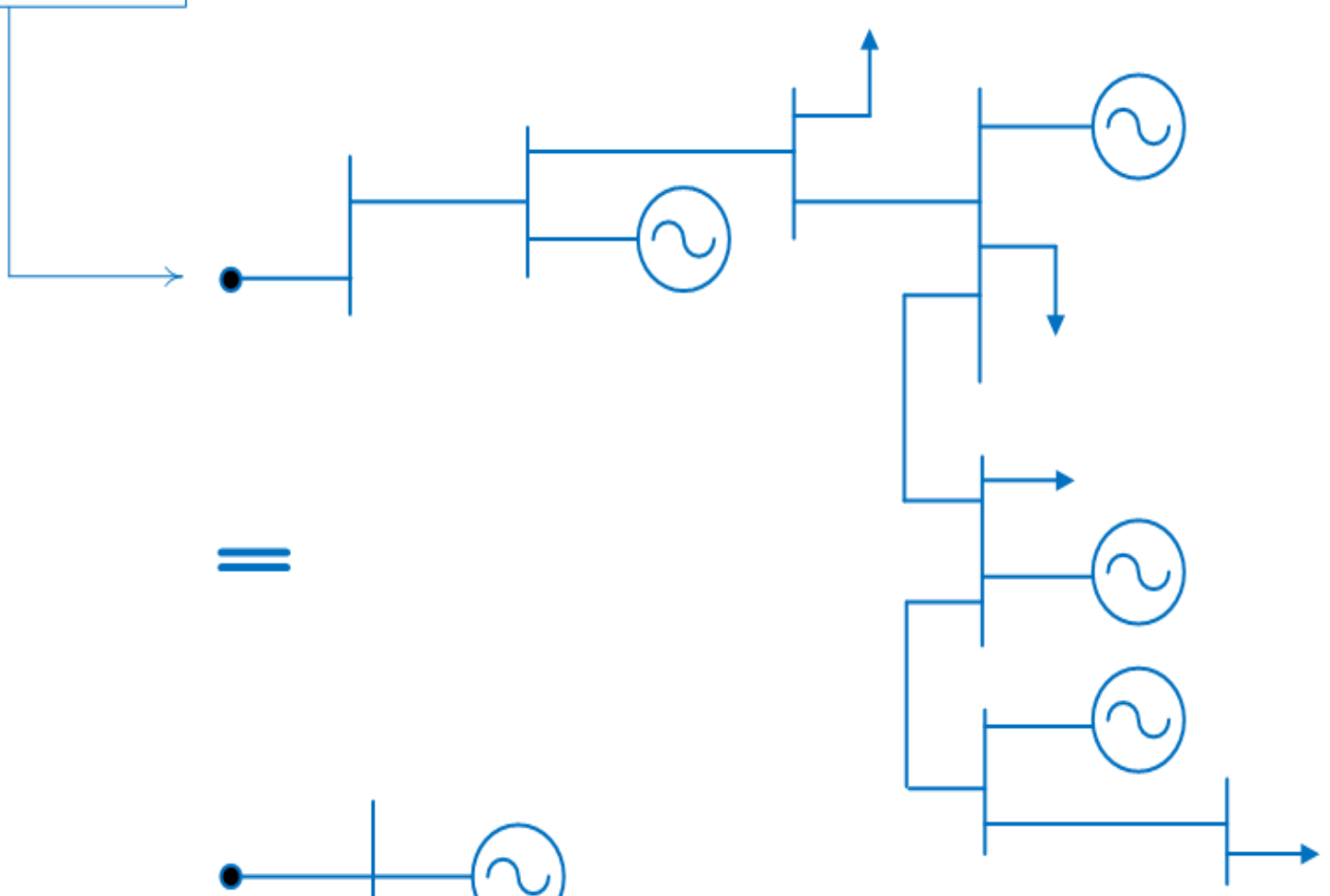

Equivalent Generator Network

Figure 2.1: Large, networked power system as a single generator.

$$
\begin{array}{ll}
\frac{2 H_{1}}{\omega_{s}} \frac{d^{2} \delta_{1}}{d t^{2}}=P_{m 1}-P_{e 1} \quad & (\text { per unit }) \\
\frac{2 H_{2}}{\omega_{s}} \frac{d^{2} \delta_{2}}{d t^{2}}=P_{m 2}-P_{e 2} & (\text { per unit })
\end{array}
$$

Adding the equations together with $\delta_{1}$ and $\delta_{2}$, which are denoted by $\delta$ since the rotors swing together, creates equation 2.20.

$$
\frac{2 H}{\omega_{s}} \frac{d^{2} \delta}{d t^{2}}=P_{m}-P_{e} \quad(\text { per unit })
$$


Where $H, P_{m}$, and $P_{e}$ in equation 2.20 are in the form of swing equation 2.12.

$$
\begin{gathered}
H=\left(H_{1}+H_{2}\right) \\
P_{m}=\left(P_{m 1}+P_{m 2}\right) \\
P_{e}=\left(P_{e 1}+P_{e 2}\right)
\end{gathered}
$$

This can then be solved to represent the combined plant dynamics.

Machines that swing together are called coherent machines. It is noted that, when both $\omega_{s}$ and $\delta$ can be expressed in electrical degrees or radians, the swing equations for coherent machines can be combined together even though the rated speeds are different. This allows stability studies that involve many machines to reduce the number of swing equations that are needed to be solved.

For any pair of non-coherent machines in a system, swing equations 2.18 and 2.19 can be written. Divide each equation by its left-handed side coefficients and subtract the resultant equations, results in equation 2.21 .

$$
\frac{d^{2} \delta_{1}}{d t^{2}}-\frac{d^{2} \delta_{2}}{d t^{2}}=\frac{\omega_{s}}{2}\left(\frac{P_{m 1}-P_{e 1}}{H_{1}}-\frac{P_{m 2}-P_{e 2}}{H_{2}}\right)
$$

Multiply each side by $\left(\frac{H_{1} H_{2}}{H_{1}+H_{2}}\right)$ and rearrange to obtain equation 2.22 . 


$$
\frac{2}{\omega_{s}}\left(\frac{H_{1} H_{2}}{H_{1}+H_{2}}\right) \frac{d^{2}\left(\delta_{1}-\delta_{2}\right)}{d^{2}}=\frac{P_{m 1} H_{2}-P_{m 2} H_{1}}{H_{1}+H_{2}}-\frac{P_{e 1} H_{2}-P_{e 2} H_{1}}{H_{1}+H_{2}}
$$

This can be simplified in the form of equation 2.12 to obtain equation 2.23 .

$$
\frac{2 H_{12}}{\omega_{s}} \frac{d^{2} \delta_{12}}{d^{2}}=P_{m 12}-P_{e 12}
$$

In equation 2.23 the relative angle $\delta_{12}$ equals $\delta_{1}-\delta_{2}$. The equivalent inertia, weighted input, and output powers are defined by the following:

$$
\begin{gathered}
H_{12}=\frac{H_{1} H_{2}}{H_{1}+H_{2}} \\
P_{m 12}=\frac{P_{m 1} H_{2}-P_{m 2} H_{1}}{H_{1}+H_{2}} \\
P_{e 12}=\frac{P_{e 1} H_{2}-P_{e 2} H_{1}}{H_{1}+H_{2}}
\end{gathered}
$$

Another application of these equations is a two-machine system having only one generator. Where the generator is (machine one) and synchronous motor is (machine two), both connected by a network of pure reactances. Whatever change occurs in one generator output is absorbed by the other generator, which can be written as the following.

$$
\begin{gathered}
P_{m 1}=-P_{m 2}=P_{m} \\
P_{e 1}=-P_{e 2}=P_{e}
\end{gathered}
$$


Where $P_{m 12}=P_{m}, P_{e 12}=P_{e}$ and then equation 2.23 reduces to

$$
\frac{2 H_{12}}{\omega_{s}} \frac{d^{2} \delta_{12}}{d t^{2}}=P_{m}-P_{e}
$$

Equation 2.22 illustrates that stability of a machine within a system is a relative property associated with its dynamic behavior with respect to the other machines of the system. In order to be stable, the angular differences between all machines must decrease after the final switching operation, such as the opening of a circuit breaker to clear a fault. This is not specifically the angle between the machine's rotor and a synchronously rotating reference axis, but more importantly its the relative angles between machines.

The aforementioned information on a two-machine system can be categorized by two types; those having one machine of finite-inertia swinging with respect to an infinite bus, and those having two finite-inertia machines swinging with respect to each other.

An infinite bus may be considered from stability purposes as a bus at which is located a machine of constant internal voltage, having zero impedance and finite inertia. The point of connection of a generator to a large power system may be regarded as such a bus. In all cases, the swing equation assumes the form of equation 2.12. The equation for $P_{e}$ is essential to this description.

\subsubsection{The Power-Angle}

In the swing equation for the generator, the input mechanical power from the prime mover $P_{m}$ is considered constant. This assumption can be made because conditions in the electrical 


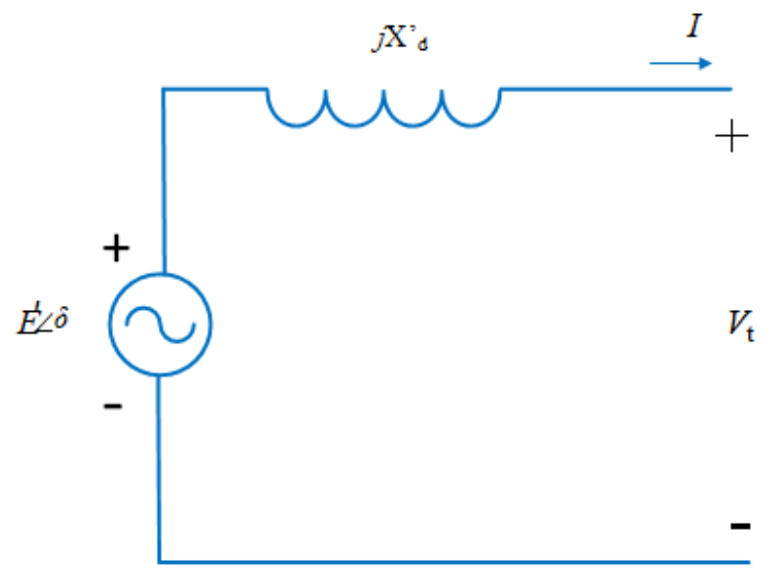

Circuit Diagram

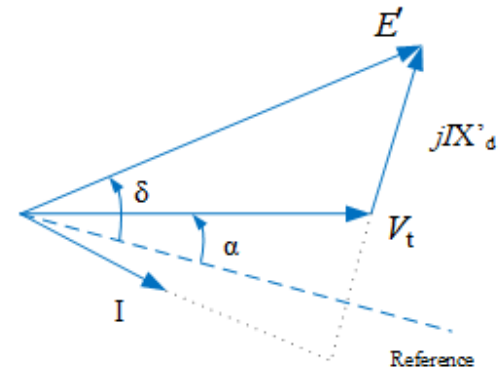

Phasor Diagram

Figure 2.2: Generator Circuit and Phasor Diagram [4]

network are not expected to change before control systems can react to an event. Since $P_{m}$ is constant in equation 2.12, the electrical power output $P_{e}$ will determine whether the rotor accelerates, decelerates, or remains at synchronous speed. When $P_{e}$ equals $P_{m}$ the machine operates at steady-state synchronous speed. When $P_{e}$ changes from this value the rotor deviates from synchronous speed. Changes in $P_{e}$ are determined by conditions in the transmission networks, distribution networks, and the loads on the system to which the generator supplies power. Electrical network disturbance or events, resulting from severe load changes, faults, or circuit breaker operations, may cause the generator output $P_{e}$ to change, in which case electromechanical transients arise.

The fundamental assumption is that the effect of the machine speed variations upon the generated voltage is negligible so that the manner in which $P_{e}$ changes is determined by the load flow equations applicable to the state of of the electrical network, and by the model 
chosen to represent the electrical behavior of the machine. Each synchronous machine is represented for transient stability studies by its transient internal voltage $E$ ' in series with the transient reactance $X_{d}^{\prime}$ as shown in Figure 2.2 in which $V_{t}$ is the terminal voltage. This corresponds to the steady-state representation in which synchronous reactance $X_{d}$ is in series with the synchronous internal voltage $E$. Since each machine must be considered relative to the system of which it is part, the phasor angle of the machine quantities are measured with respect to a common reference.

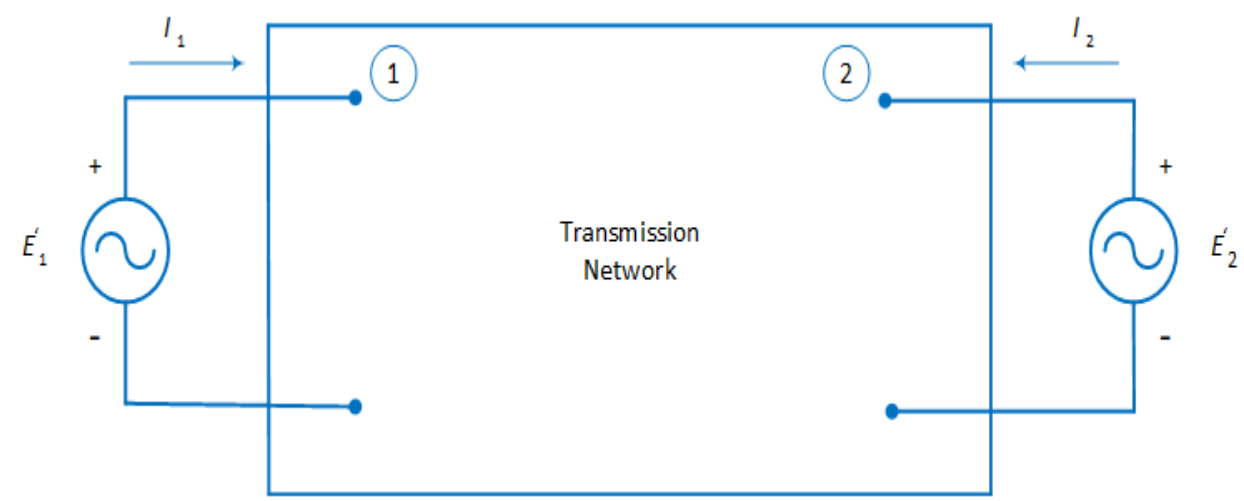

Figure 2.3: Schematic Diagram Transient Reactances [10]

Figure 2.3 represents a generator supplying power through a transmission system to a receiving end at bus 2 . The schematic represents the transmission system of linear passive components and includes the transient reactance of the generator. The voltage $E_{1}^{\prime}$ represents the transient internal voltage of the generator at bus 1 . The voltage $E_{2}^{\prime}$ at the receiving end represents an infinite bus, or as the transient internal voltage of a synchronous motor whose transient reactance is included in the network.

The elements of the bus admittance matrix for the network reduced to two nodes in 
addition to the reference node, is equation 2.28.

$$
Y_{\text {bus }}=\left[\begin{array}{ll}
Y_{11} & Y_{12} \\
Y_{21} & Y_{22}
\end{array}\right]
$$

Complex power into the bus is given by equation 2.29 .

$$
P_{k}+j Q_{1}=V_{k}^{*} \sum_{n=1}^{N} Y_{k n} V_{n}
$$

Letting $k$ and $N$ equal 1 and 2, respectively, and substituting $E_{2}{ }_{2}$ for $V$ can be written as equation 2.30 at bus 1 .

$$
P_{1}+j Q_{1}=E^{\prime}{ }_{1}\left(Y_{11} E^{\prime}{ }_{1}\right)^{*}+E^{\prime}{ }_{1}\left(Y_{12} E_{2}^{\prime}\right)^{*}
$$

Where the following internal voltages and admittances apply.

$$
\begin{gathered}
E_{1}^{\prime}=\left|E_{1}^{\prime}\right| \angle \delta_{1}: E_{2}^{\prime}=\left|E_{2}^{\prime}\right| \angle \delta_{2} \\
Y_{11}=G_{11}+j B_{11}: Y_{12}=\left|Y_{12}\right| \angle \theta_{12}
\end{gathered}
$$

Since in the solution of the swing equation only real power is involved we have equation 2.31 .

$$
\begin{gathered}
P_{1}=\left|E^{\prime}{ }_{1}\right|^{2} G_{11}+\left|E^{\prime}{ }_{1}\right| E_{2}{ }_{2}\left|Y_{11}\right| \cos \left(\delta_{1}-\delta_{2}-\theta_{12}\right) \\
Q_{1}=-\left|E^{\prime}{ }_{1}\right|^{2} B_{11}+\left|E^{\prime}{ }_{1}\right| E_{2}{ }_{2}\left|Y_{12}\right| \sin \left(\delta_{1}-\delta_{2}-\theta_{12}\right)
\end{gathered}
$$

Similar equations apply at bus 2 by interchanging the above subscripts in equations 2.31 and 2.32 . 
If we define the following,

$$
\delta=\delta_{1}-\delta_{2}
$$

and define a new angle $\gamma$ such that,

$$
\gamma=\theta_{12}-\frac{\pi}{2}
$$

we obtain Equations 2.33 and 2.34.

$$
\begin{gathered}
P_{1}=\left|E^{\prime}{ }_{1}\right|^{2} G_{11}+\left|E^{\prime}{ }_{1}\right| E^{\prime}{ }_{2}\left|Y_{12}\right| \sin (\delta-\gamma) \\
Q_{1}=-\left|E{ }_{1}\right|^{2} B_{11}-\left|E{ }_{1}\right| E{ }_{2}\left|Y_{12}\right| \cos (\delta-\gamma)
\end{gathered}
$$

The equation 2.31 can be rewritten as Equation 2.35, which is the Power Angle Equation.

$$
P_{e}=P_{c}+P_{\text {max }} \sin (\delta-\gamma)
$$

Where $P_{c}$ and $P_{\text {max }}$ are the following.

$$
P_{c}=\left|E_{1}^{\prime}\right|^{2} G_{11}: P_{\max }=\left|E_{1}^{\prime}\right|\left|E_{2}^{\prime}\right|\left|Y_{12}\right|
$$

Since $P_{1}$ represents the electrical power output of the generator, it has been replaced by $P_{e}$ in the power-angle equation 2.35; its graph as a function $\delta$ is called the power-angle curve. The parameters $P_{c}, P_{\max }$, and $\gamma$ are constants for a given network configuration and constant voltage magnitudes $\left|E^{\prime}{ }_{1}\right|$ and $\left|E^{\prime}{ }_{2}\right|$. For a purely reactive network, which is a common assumption when conducting power system analysis, all the elements of the $Y_{b u s}$ are susceptances, where both $G_{11}$ and $\gamma$ are zero. 
The power-angle equation for a pure reactance network then becomes equation 2.37.

$$
P_{e}=P_{\max } \sin \delta
$$

And $P_{\max }$ can be written as equation 2.38 .

$$
P_{\max }=\frac{\left|E_{1}^{\prime}\right|\left|E_{2}^{\prime}\right|}{X}
$$

Where $X$ is the transfer reactance between $E_{1}^{\prime}$ and $E_{2}^{\prime}$.

The swing equation 2.15 for the machine can be written as equation 2.39 , where $H$ is in megajoules per megavoltampere, $f$ is the electrical frequency, and rotor angle $\delta$ is in electrical degrees.

$$
\frac{H}{180 f} \frac{d^{2} \delta}{d t^{2}}=P_{m}-P_{\max } \sin \delta \quad(\text { per unit })
$$

The graphical plot of the power-angle equation is shown in Fig. 2.4.

\subsubsection{Synchronizing Power Coefficients}

The requirement for a synchronous generator is that it will not lose synchronism when small temporary changes occur in the output of a machine. To highlight this requirement, for a fixed mechanical input power $P_{m}$, consider small incremental changes in the operating point parameters. Stevenson [4] provides excellent discussion of units and dimensional analysis of synchronizing power coefficients.

$$
\delta=\delta_{0}+\delta_{\Delta}: P_{e}=P_{e 0}+P_{e \Delta}
$$




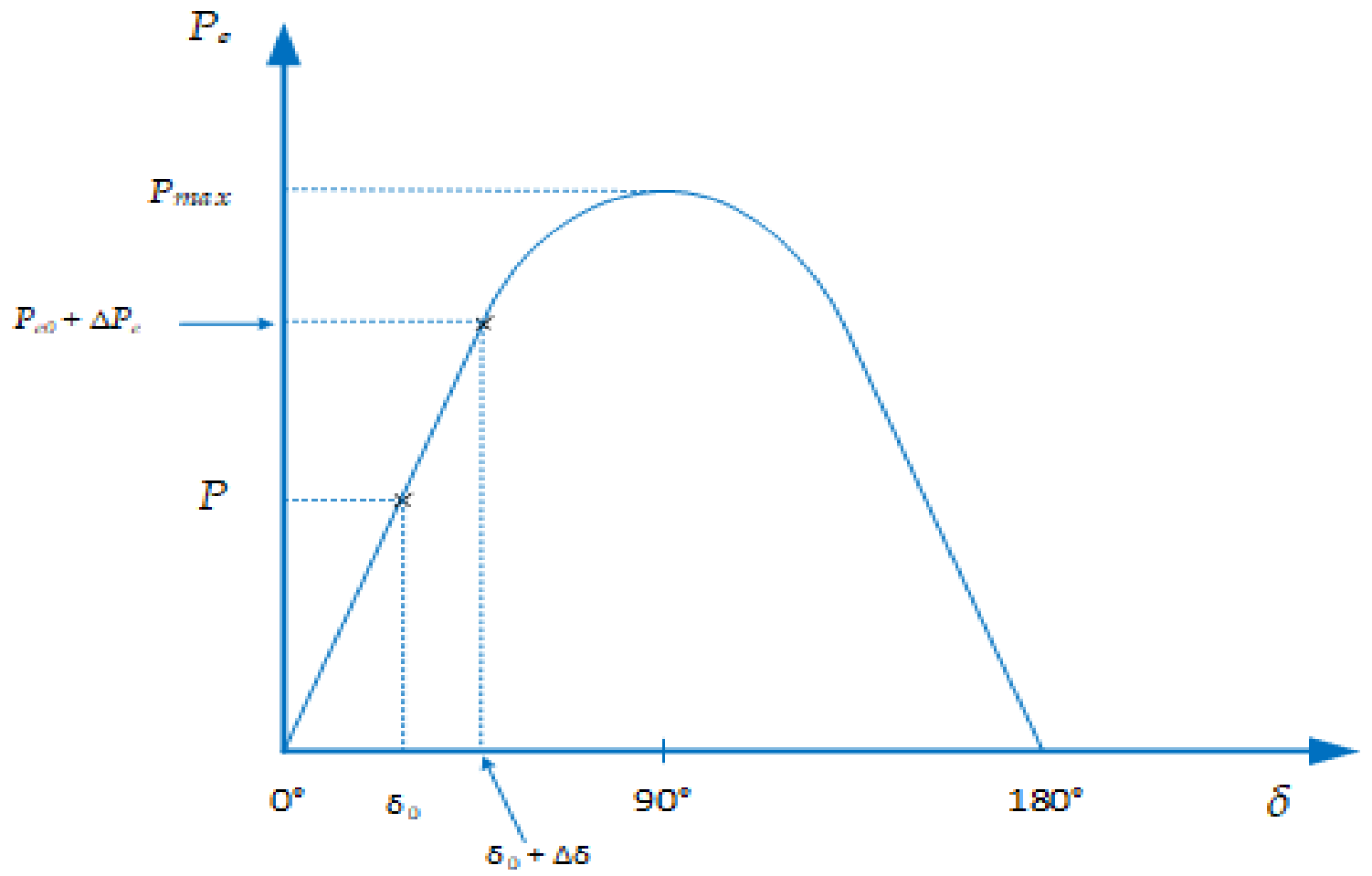

Figure 2.4: Power-Angle Equation Plot [4]

The subscript zero denotes the steady-state operating point values and the subscript zero identifies the incremental variations from those values. Substituting equation 2.40 into 2.35, to obtain the power-angle equation for a general two-machine system in the form of of the following equations.

$$
\begin{gathered}
P_{e 0}+P_{e \Delta}=P_{\max } \sin \left(\delta_{0}+\delta_{\Delta}\right) \\
P_{e 0}+P_{e \Delta}=P_{\max }\left(\sin \delta_{0} \cos \delta_{\Delta}+\cos \delta_{0} \sin \delta_{\Delta}\right)
\end{gathered}
$$

$\delta_{\Delta}$ is a small incremental displacement from $\delta_{0}$.

$$
\sin \delta_{\Delta} \cong \delta_{\Delta} \text { and } \cos \delta_{\Delta} \cong 1
$$


Then equation 2.42 becomes 2.44

$$
P_{e 0}+P_{e \Delta}=P_{\max } \sin \delta_{0}+\left(P_{\max } \cos \delta_{0}\right) \delta_{\Delta}
$$

where strict equality is now used. At the initial operating point $\delta_{0}$,

$$
P_{m}=P_{e_{0}}=P_{\max } \sin \delta_{0}
$$

and from the equations 2.44 and 2.45 it follows that

$$
P_{m}-\left(P_{e_{0}}+P_{e \Delta}\right)=-\left(P_{\max } \cos \delta_{0}\right) \delta_{\Delta}
$$

Substituting the incremental variables of equation 2.40 into the basic swing equation 2.12, we obtain equation 2.47 .

$$
\frac{2 H}{\omega_{s}} \frac{d^{2}\left(\delta_{0}+\delta_{\Delta}\right)}{d^{2}}=P_{m}-\left(P_{e 0}+P_{e_{\Delta}}\right)
$$

Replacing the right-hand side of this equation by 2.46 and transposing terms, we obtain equation 2.48 ,

$$
\frac{2 H}{\omega_{s}} \frac{d^{2} \delta_{\Delta}}{d t^{2}}+\left(P_{\max } \cos \delta_{0}\right) \delta_{\Delta}=0
$$

where $\delta_{0}$ is a constant value. Noting that $P_{\max } \cos \delta_{0}$ is the slope of the power-angle curve at the angle $\delta_{0}$, we denote the slope as $S_{p}$ and define it as equation 2.49.

$$
S_{p}=\left.\frac{d P_{e}}{d \delta}\right|_{\delta=\delta_{0}}=P_{\max } \cos \delta_{0}
$$

Where $S_{p}$ is called the synchronizing power coefficient. When $S_{P}$ is used in equation 2.48, the swing equation governing the incremental rotor-angle variations may be written in the form of equation 2.50 .

$$
\frac{d^{2} \delta_{\Delta}}{d t^{2}}+\frac{\omega_{s} S_{p}}{2 H} \delta_{\Delta}=0
$$


This is a linear, second-order differential equation, the solution to which depends upon the algebraic sign of $S_{p}$. When $S_{p}$ is positive, the solution of $\delta_{\Delta}(t)$ corresponds to that of simple harmonic motion where the oscillations are of an undamped sinusoid of angular frequency $\omega_{n}$. The equation of simple harmonic motion is the following equation.

$$
\frac{d^{2} x}{d t^{2}}+\omega^{2} x=0
$$

This has a general solution with constants $A$ and $B$ determined by initial conditions.

$$
x(t)=A \cos \omega_{n} t+B \sin \omega_{n} t
$$

When $S_{p}$ is negative, the solution $\delta \Delta(t)$ increases exponentially without limit.

The angular frequency of undamped oscillations is given by equation 2.51 .

$$
\omega_{n}=\sqrt{\frac{\omega_{s} S_{p}}{2 H}} \quad(\text { elec } \mathrm{rad} / \mathrm{s})
$$

Which corresponds to the frequency of oscillation given by equation 2.52 .

$$
f_{n}=\frac{1}{2 \pi} \sqrt{\frac{\omega_{s} S_{p}}{2 H}} \quad(H z)
$$

\subsubsection{Equal-Area Criterion}

The swing equations developed in the power angle equations are nonlinear in nature. Formal solution of such equations can not be explicitly found. Even the case of a single machine swinging with respect to an infinite bus is very difficult to obtain literal-form solutions and therefore digital computer methods are normally used. To examine the stability of a twomachine system without solving the swing equation, a direct approach is possible. Kimbark 
[2] and Stevenson [4] provide excellent discussion of units and dimensional analysis of equal-area criterion of stability.

Consider a Single Machine Infinite Bus (SMIB) system, as shown in Figure 2.5. At point P close to the bus number one, a three-phase fault occurs and it is cleared by circuit breaker A after a short period. Therefore, the effective transmission system is unaltered except while the fault is on. The short circuit caused by the fault is effectively at the bus and so the electrical power from the generator is zero until the fault is cleared. The physical conditions before, during, and after the fault can be understood by analysing the power-angle curves in Figure 2.6.

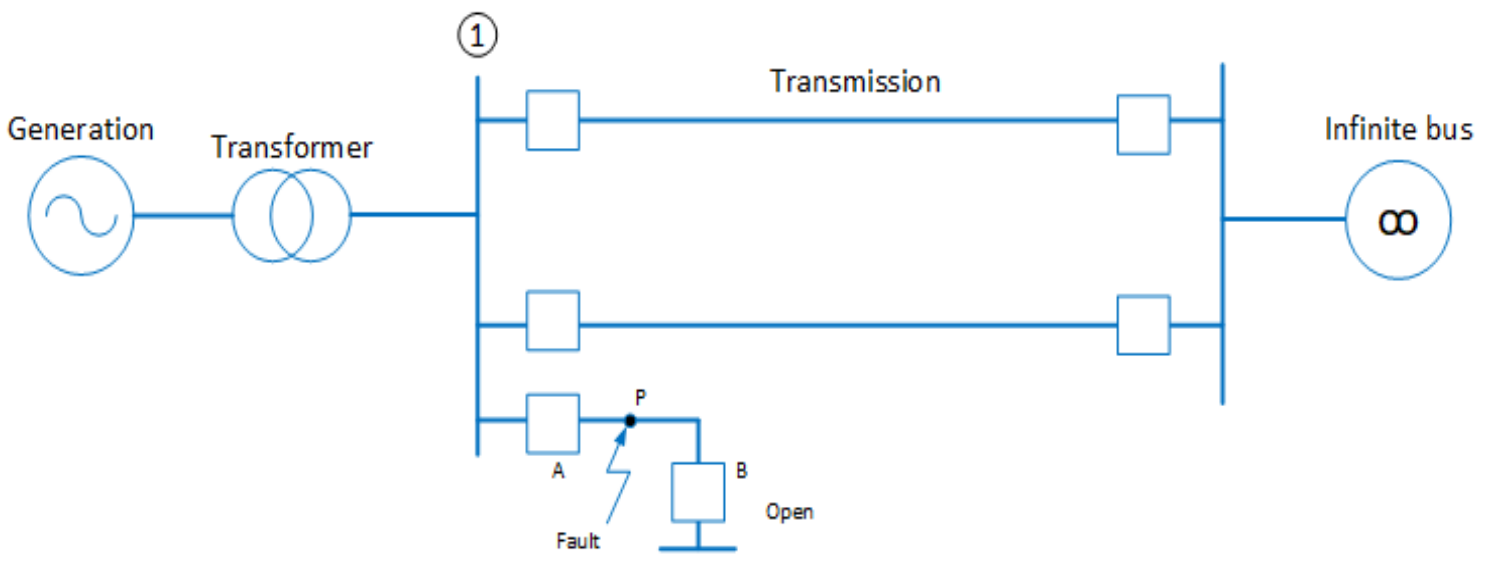

Figure 2.5: SMIB System with Short Transmission line [4]

Originally the generator is operating at synchronous speed with a rotor angle of $\delta_{0}$ and the input mechanical power $P_{m}$ equals the output electrical power $P_{e}$ as shown at point $a$ in Figure 2.6a. When the fault occurs at $\mathrm{t}=0$, the electrical power output is suddenly zero while the input mechanical power is unaltered as shown in Figure 2.6b. The difference in 
power must be accounted for by a rate of change of stored kinetic energy in the rotor masses. This can be accomplished only by an increase in speed, which results from the constant accelerating power $P_{m}$. If we denote the time to clear the fault $t_{c}$, then for time $t$ less than $t_{c}$ the acceleration is constant and is given by equation 2.53 .

$$
\frac{d^{2} \delta}{d t^{2}}=\frac{\omega_{s}}{2 H} P_{m}
$$

While the fault is on, the velocity increase above synchronous speed is found by integrating equation 2.53 to equation 2.54 .

$$
\frac{d \delta}{d t}=\int_{0}^{t} \frac{\omega_{s}}{2 H} P_{m} d t=\frac{\omega_{s}}{2 H} P_{m} t
$$

A further integration with respect to time yields equation 2.55 for the rotor angle position.

$$
\delta=\frac{\omega_{s} P_{m}}{4 H} t^{2}+\delta_{0}
$$

Equations 2.54 and 2.55 show that the velocity of the rotor relative to the synchronous speed increases linearly with time while the rotor angle advances from $\delta_{0}$ to the angle at clearing $\delta_{c}$; that is, Figure 2.6 the angles $\delta$ goes from $b$ to $c$. At the instant of the fault clearing, the increase in rotor speed and the angle of separation between the generator and the infinite bus are given, respectively, by equation 2.56 and equation 2.57 .

$$
\begin{gathered}
\left.\frac{d \delta}{d t}\right|_{t=t_{c}}=\frac{\omega_{s} P_{m}}{2 H} t_{c} \\
\left.\delta(t)\right|_{t=t_{c}}=\frac{\omega_{s} P_{m}}{4 H} t_{c}^{2}+\delta_{0}
\end{gathered}
$$

When the fault is cleared at the angle $\delta_{c}$, the electrical power output immediately increases to a value corresponding to point $d$ on the power-angle curve. At $d$ the electrical 


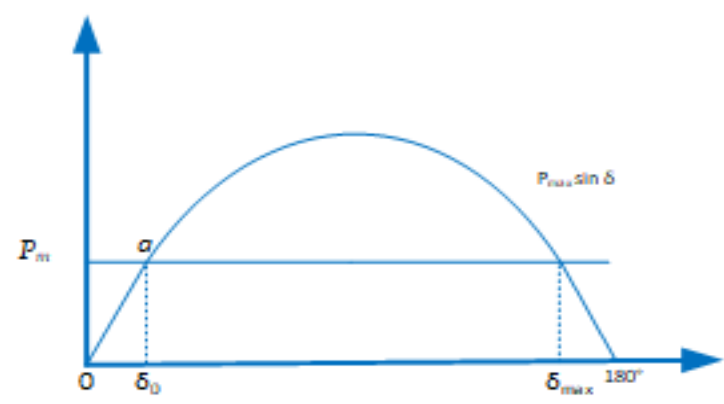

(a)

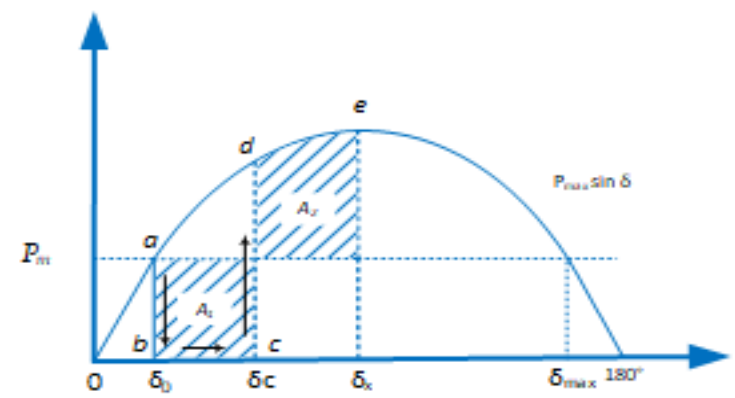

(b)

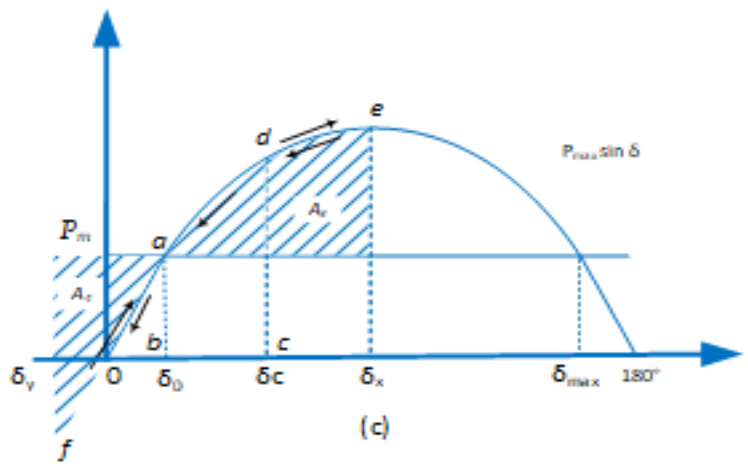

Figure 2.6: Power-Angle Curves [4]

power output exceeds the mechanical power input and thus the acceleration is negative. As a consequence, the rotor slows down as $P_{e}$ goes from $d$ to $e$ in Figure 2.6c. At $e$ the rotor speed is again synchronous although the rotor angle has advanced to $\delta_{x}$. The angle $\delta_{x}$ is determined by the fact that areas $A_{1}$ and $A_{2}$ must be equal. The accelerating power at $e$ is still negative (retarding), and so the rotor cannot remain at synchronous speed but must 
continue to slow down. The relative velocity is negative and the rotor angle moves back from $\delta_{x}$ at $e$ along the power-angle curve of Figure $2.6 \mathrm{c}$ to point $a$ at which the rotor speed is less than synchronous. From $a$ to $f$ the mechanical power exceeds the electrical power and rotor increases speed again until it reaches synchronism at $f$. Point $f$ is located so that areas $A_{3}$ and $A_{4}$ are equal. In the absence of damping the rotor would continue to oscillate in the sequence f-a-e, e-a-f, etc., with synchronous speed occurring at $e$ and $f$.

In the system where the machine is swinging with respect to an infinite bus, the use of the principle of equality of areas, called the equal-area criterion, determines the stability of the system under transient conditions without solving the swing equation. Although not applicable to multimachine systems, the method helps in understanding how certain factors influence the transient stability of any system.

The derivation of the equal-area criterion is made for one machine and an infinite bus although the method can be adopted to two-machine systems. The swing equation for the machine connected to the bus is equation 2.58 .

$$
\frac{2 H}{\omega_{s}} \frac{d^{2} \delta}{d t^{2}}=P_{m}-P_{e}
$$

Define the angular velocity of the rotor relative to synchronous speed as equation 2.59.

$$
\omega_{r}=\frac{d \delta}{d t}=\omega-\omega_{s}
$$

Differentiating equation 2.59 with respect to $t$ and substituting in equation 2.58 we obtain equation 2.60 .

$$
\frac{2 H}{\omega_{s}} \frac{d \omega_{r}}{d t}=P_{m}-P_{e}
$$


When the rotor speed is synchronous, $\omega$ equals $\omega_{s}$ and $\omega_{r}$ is zero. Multiplying both sides of equation 2.60 by $\omega_{r}=\frac{d \delta}{d t}$ we obtain equation 2.61 .

$$
\frac{H}{\omega_{s}} 2 \omega_{r} \frac{d \omega_{r}}{d t}=\left(P_{m}-P_{e}\right) \frac{d \delta}{d t}
$$

The left-hand side of the equation can be rewritten to provide equation 2.62 .

$$
\frac{H}{\omega_{s}} \frac{d\left(\omega_{r}^{2}\right)}{d t}=\left(P_{m}-P_{e}\right) \frac{d \delta}{d t}
$$

Multiplying by $d t$ and integrating, we obtain equation 2.63 .

$$
\frac{H}{\omega_{s}}\left(\omega_{r_{2}}^{2}-\omega_{r_{1}}^{2}\right)=\int_{\delta_{1}}^{\delta_{2}}\left(P_{m}-P_{e}\right) d \delta
$$

The subscripts for the $\omega_{r}$ terms corresponds to the $\delta$ limits, where the rotor speed $\omega_{r_{1}}$ corresponds to angle $\delta_{1}$ and $\omega_{r_{2}}$ corresponds to $\delta_{2}$. Since $\omega_{r}$ represents the departure of the rotor speed from synchronous speed, we can determine that if the rotor speed is synchronous at $\delta_{1}$ and $\delta_{2}$, then correspondingly, $\omega_{r_{1}}=\omega_{r_{2}}=0$. Under this condition equation 2.63 becomes equation 2.64 .

$$
\int_{\delta_{1}}^{\delta_{2}}\left(P_{m}-P_{e}\right) d \delta=0
$$

This equation applies to any two points $\delta_{1}$ and $\delta_{2}$ on the power-angle diagram, provided they are at which the rotor speed is synchronous. In Figure $2.6 \mathrm{~b}$ two such points are $a$ and $e$ corresponding to $\delta_{0}$ and $\delta_{x}$. If we perform the integration in steps, we can write the following.

$$
\int_{\delta_{0}}^{\delta_{c}}\left(P_{k}-P_{e}\right) d \delta+\int_{\delta_{c}}^{\delta_{x}}\left(P_{m}-P_{e}\right) d \delta=0
$$

or

$$
\int_{\delta_{0}}^{\delta_{c}}\left(P_{k}-P_{e}\right) d \delta=\int_{\delta_{c}}^{\delta_{x}}\left(P_{m}-P_{e}\right) d \delta
$$


The integral applies to the fault period while the right integral corresponds the immediate post fault period up to the point of maximum swing $\delta_{x}$. In Figure $2.6 \mathrm{~b} P_{e}$ is zero during the fault. The shaded area $A_{1}$ is given by the left-hand side equation 2.66 and the shaded area $A_{2}$ is given by the right-hand side. So the two areas $A_{1}$ and $A_{2}$ are equal.

Since the rotor speed is synchronous at $\delta_{x}$ and also at $\delta_{y}$ in Figure $2.6 \mathrm{c}$ the same reasoning shows that $A_{3}$ equals $A_{4}$. The areas $A_{1}$ and $A_{4}$ are directly proportional to the increase in kinetic energy of the rotor while it is decelerating. This can be seen by inspection of both sides of equation 2.63. The equal-area criterion states that whatever kinetic energy is added to the rotor following a fault must be removed after the fault to restore the rotor to synchronous speed.

The shaded area $A_{1}$ is dependent upon the time taken to clear the fault. If there is a delay in clearing, the angle $\delta_{c}$ is increased; likewise the area $A_{1}$ increases and the equal-area criterion requires that area $A_{2}$ also increase to restore the rotor to synchronous speed at a larger angle of maximum swing $\delta_{x}$. If the delay in clearing is prolonged where the rotor angle $\delta$ swings beyond the angle $\delta_{\max }$ in Figure 2.6 then the rotor speed at that point on the power-angle curve is above synchronous speed when positive accelerating power is again encountered. Under the influence of this positive accelerating power the angle $\delta$ will increase without limit and instability results. Therefore there is a critical angle for clearing the fault in order to satisfy the requirements of the equal-area criterion for stability. This angle, called the critical clearing angle $\delta_{c r}$ is illustrated in Figure 2.7. The corresponding critical time for removing the fault is called the critical clearing time $\delta_{c r}$. 


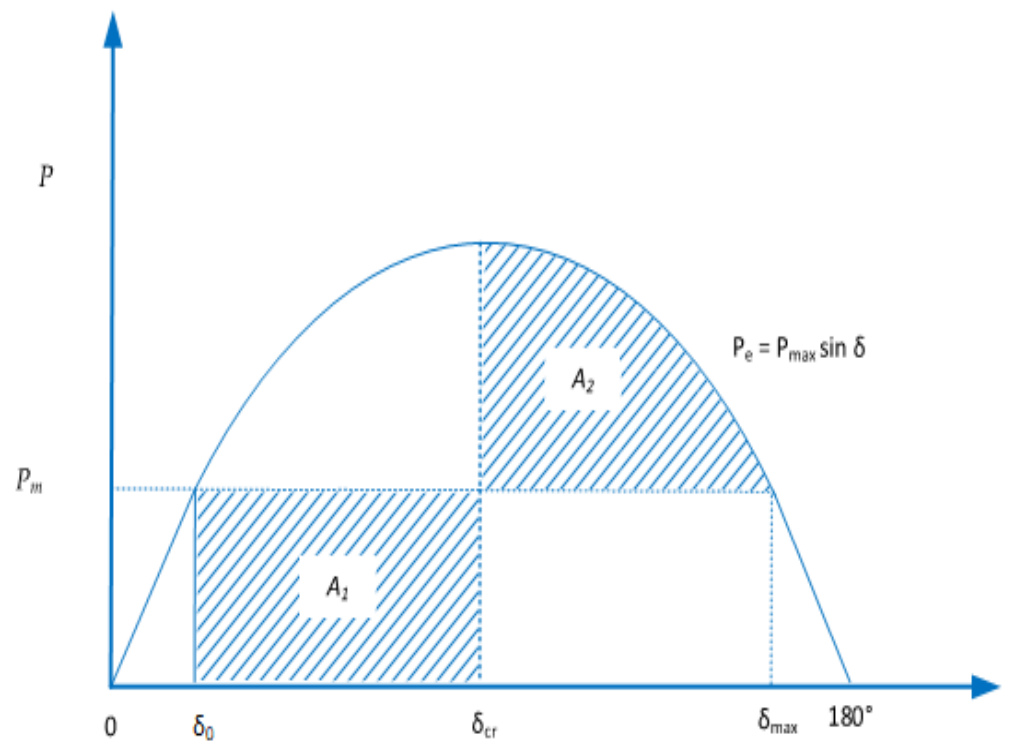

Figure 2.7: Power-Angle Curves with critical-clearing [4]

From Figure 2.7, both the critical clearing angle and the critical clearing time can be calculated as follows. The rectangle area $A_{1}$ in equation 2.67 .

$$
A_{1}=\int_{\delta_{0}}^{\delta_{c r}} P_{m} d \delta=P_{m}\left(\delta_{c r}-\delta_{0}\right)
$$

While the area for $A_{2}$ is equation

$$
\begin{array}{r}
A_{2}=\int_{\delta_{c r}}^{\delta_{\max }}\left(P_{\max } \sin \delta-P_{m}\right) d \delta \\
A_{2}=P_{\text {max }}\left(\cos \delta_{c r}-\cos \delta_{\text {max }}\right)-P_{m}\left(\delta_{\text {max }}-\delta_{c r}\right)
\end{array}
$$

equating the expressions for $A_{1}$ and $A_{2}$, and transposing terms, yields equation 2.69.

$$
\cos \delta_{c r}=\left(\frac{P_{m}}{P_{\max }}\right)\left(\delta_{\max }-\delta_{0}\right)+\cos \delta_{\max }
$$

We see from the sinusoidal power-angle curve that $\delta_{\max }$ is equation 2.70 .

$$
\delta_{\max }=\pi-\delta_{0} \quad(\text { elec } \mathrm{rad})
$$


And that $P_{m}$ is equation 2.71 .

$$
P_{m}=P_{\max } \sin \delta
$$

Substituting for $\delta_{\max }$ and $P_{m}$ in equation 2.69, simplifying the result and solving for $\delta_{c r}$, we obtain equation 2.72 for the critical clearing angle.

$$
\delta_{c r}=\cos ^{-1}\left[\left(\pi-2 \delta_{0}\right) \sin \delta_{0}-\cos \delta_{0}\right]
$$

The value for $\delta_{c r}$ calculated from this equation, when substituted for the right-hand side of equation 2.57, yields equation 2.73 .

$$
\delta_{c r}=\frac{\omega_{s} P_{m}}{4 H} t_{c r}^{2}+\delta_{0}
$$

From which is found equation 2.74 for the critical clearing time.

$$
t_{c r}=\sqrt{\frac{4 H\left(\delta_{c r}-\delta_{0}\right)}{\omega_{s} P_{m}}}
$$

Although the equal-area criterion can be applied only for the case of two machines or one machine and an infinite bus, it is very useful means for beginning to see what happens when a fault occurs and helpful in understanding transient stability.

\subsubsection{Why are there stability studies}

Synchronous machines do not easily fall out of step under normal conditions. Stability studies are needed to understand the stability limit of synchronous machines to determine whether the machines remain in synchronism after a disturbance.

Power-system stability is a term applied to alternating-current electric power systems, denoting a condition in which the various synchronous machines of the given system remain 
in synchronism, or "in step," with each other [2]. Conversely, instability denotes a condition involving a loss of synchronism, or falling "out of step."

Stability can be formally defined as follows: Stability when used with reference to a power system, is that attribute of the system, or part of a system, which enables it to develop restoring forces between the elements thereof, equal to or greater than disturbing forces so as to restore a state of equilibrium between the elements [11].

Successful operation of a power system depends largely on an electrical power system's ability to provide reliable and uninterrupted service to the loads [5]. The reliability of the power supply implies much more than being available. Ideally, the loads must be fed at constant voltage and frequency at all times.

The stability limit for a system with synchronous machines can be considered the same as the power limit, and is defined as: A stability limit is the maximum power flow possible through some point in the system when the entire system or part of the system to which the stability limit refers is operating with stability [11]. When a synchronous machine loses synchronism or "falls out of step" with the rest of the system, its rotor runs at a higher or lower speed than required to generate voltages at system frequency [12]. The slip between the rotating stator field (corresponding to system frequency) and the rotor field results in large fluctuations in the machine power output, current and voltage; this causes the protection system to isolate the unstable machine from the system.

Instability in a power system may be manifested in different ways depending on the 
system configuration and operating mode. Traditionally, the stability problem has been one of maintaining synchronous operation. Since power systems rely on synchronous machines for generation of electrical power, a necessary condition is that all the synchronous machines remain in synchronism. This aspect of stability is influenced by the dynamics of generator rotor angles and power-angle relationships.

Rotor angle stability is the ability of interconnected synchronous machines of a power system to remain in synchronism. Voltage stability is the ability of a power system to maintain acceptable voltages at all buses in the system under normal operating conditions and after being subjected to a disturbance. For the voltage to be stable, the synchronous machines must run in synchronism. The long-term and mid-term stability are relatively new to the literature on power system stability [12]. Long-term stability is associated with the slower and longer-duration phenomena that accompany large-scale system upsets and on the resulting large, and sustained mismatches between generation and consumption of active and reactive power. In mid-term stability, the focus is on synchronizing power oscillations between machines, including the effects of some of the slower phenomena and possibly large voltage or frequency excursions [12].

Stability studies are usually classified into three types depending upon the nature and magnitude of the disturbance. These are transient, dynamic, and steady-state stability studies. In all stability studies, the objective is to determine whether or not the rotors of the machines being perturbed return to constant speed operation. 
Transient stability studies constitute the major analytical approach to the study of powersystem electromechanical behavior. Transient stability studies are aimed at determining if the system will remain in synchronism following major disturbances such as transmission system faults, sudden load changes, loss of generating units, or line switching. Such studies began more than 70 years ago, but were confined to consideration of dynamic problems of not more than two machines [5]. Present-day powers systems are vast, heavily interconnected systems with many hundreds of machines which can dynamically interact through the medium of their extra-high and ultra-high voltage networks.

Dynamic and steady-state stability studies are less extensive in scope and involve one or just a few machines undergoing gradual changes in operating conditions. Both dynamic and steady-state stability studies concern the stability of the locus of essentially steady-state operating points of the system. Dynamic and steady-state differ in the degree of detail used to model the machines. In dynamic stability studies, the excitation system and turbinegoverning system are represented along with synchronous machine models, which provide for flux-linkage variation in the machine air-gap [5]. Steady-state stability problems use simple generator models, which treat the generator as a constant voltage source. The solution technique of dynamic and steady-state stability problems is to examine the stability of the system under incremental variations about an equilibrium point. The nonlinear differential and algebraic equations for a system can be replaced by a set of linear equations, which are then solved by methods of linear analysis to determine whether a machine remains in synchronism following small changes from the operating point. 
Transient stability studies involve large disturbances, which do not allow a linearization process to be used. Non-linear differential and algebraic equations must be solved by direct methods or by iterative step-by-step procedures. Transient stability problems can be subdivided into first-swing and multiswing stability problems. First-swing stability is based on a simple generator model without representation of the control systems. Usually the time period under study is the first second following a system fault. If the machines of the system are found to remain in synchronism within the first second, the system is stable. Multiswing stability problems extend over a longer study period and consider the effects of generator control systems. Machine models are much more complex in transient stability studies [5].

\subsubsection{Small-Signal Stability}

A synchronous machine, when perturbed, will have several modes of oscillations with respect to the rest of the system. There are cases where coherent groups of machines oscillate with respect to other groups of machines. The oscillations cause fluctuations in bus voltages, system frequencies, and tie-line power flows. If two similar systems are connected together through a tie-line, it is evident that they can vary in speed together as one machine or that the two systems can oscillate against each other about a point in the middle of the tie or that both modes of oscillation can occur simultaneously [13]. It is important that these oscillations should be small in magnitude and should be damped if the system is to be stable in the sense of the definition of stability [5].

When an electric power system is subjected to a small disturbance it may be temporary 
or permanent. If the system is stable, we would expect that for a temporary disturbance the system would return to its initial state, while a permanent disturbance would cause the system to acquire a new operating state after a transient period. In either case, synchronism should not be lost. Under normal operating conditions a power system is subjected to small disturbances at random. It is important that synchronism not be lost under these conditions. Thus system behavior is a measure of dynamic stability as the system adjusts to small perturbations. Both Kundur and Anderson provide an excellent discussion of units and dimensional analysis of the small-signal stability [12][5].

With electric power systems, the change in electrical torque of a synchronous machine following a perturbation can be evaluated into torque components. Where in equation 2.75

$$
\Delta T_{e}=T_{s} \Delta \delta+T_{D} \Delta \omega
$$

$T_{s} \Delta \delta$ is the component of torque change in phase with the rotor angle perturbation $\Delta \delta$. This is referred to as the synchronizing torque component, and $T_{s}$ is the synchronizing torque coefficient.

$T_{D} \Delta \omega$ is the component of torque in phase with the speed deviation $\Delta \omega$, which is referred to as the damping torque component, and $T_{D}$ is the damping torque coefficient.

System stability depends on the existence of both components of torque for each synchronous machine. Lack of synchronizing torque results in instability through aperiodic drift in rotor angle, and the lack of sufficient damping torque results in oscillatory instability. Figure 2.8 illustrates the rotor stability phenomena of a small-disturbance response with 
a generator connected to a large power system. In part (a), in the absence of automatic voltage regulation (i.e., constant field voltage) the instability is due to lack of sufficient synchronizing torque. This results in instability through a non-oscillatory mode. With continuously acting voltage regulation, the small-disturbance stability problem is corrected with sufficient damping of the system oscillations, as illustrated in part (b). Instability is normally through oscillations of increasing amplitude.

The criteria for small-disturbance is that the system can be linearized about a quiescent operating state. The power-angle relationship for a synchronous machine connected to an infinite bus obeys a sine law seen in equations 2.37 and 2.38 , which is illustrated in equation 2.76 .

$$
P_{e}=\frac{\left|E_{1}^{\prime}\right|\left|E_{2}^{\prime}\right|}{X} \sin \delta=P_{\max } \sin \delta
$$

From equations 2.40 and 2.44 it shows that

$$
\begin{gathered}
P_{e}=P_{e 0}+P_{e \Delta} \\
P_{e 0}+P_{e \Delta}=P_{\text {max }} \sin \delta_{0}+\left(P_{\text {max }} \cos \delta_{0}\right) \delta_{\Delta}
\end{gathered}
$$

for small perturbations the change in power is approximately proportional to the change in angle from equation 2.77, where the quantities, in parenthesis is the slope of the power angle curve at $\delta_{0}$.

$$
P_{e \Delta}=\left(P_{\max } \cos \delta_{0}\right) \delta_{\Delta}
$$

$S_{p}$ from equation 2.49 was defined to be the synchronizing power coefficient.

$$
S_{p}=\left.\frac{d P_{e}}{d \delta}\right|_{\delta=\delta_{0}}=P_{\max } \cos \delta_{0}
$$




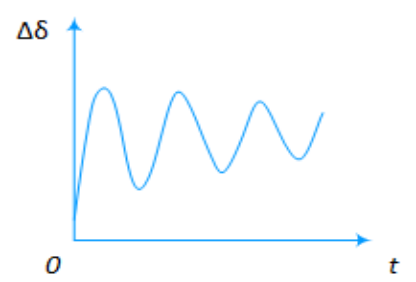

Stable

- Positive $T_{5}$

- Positive $T_{\mathrm{D}}$

0
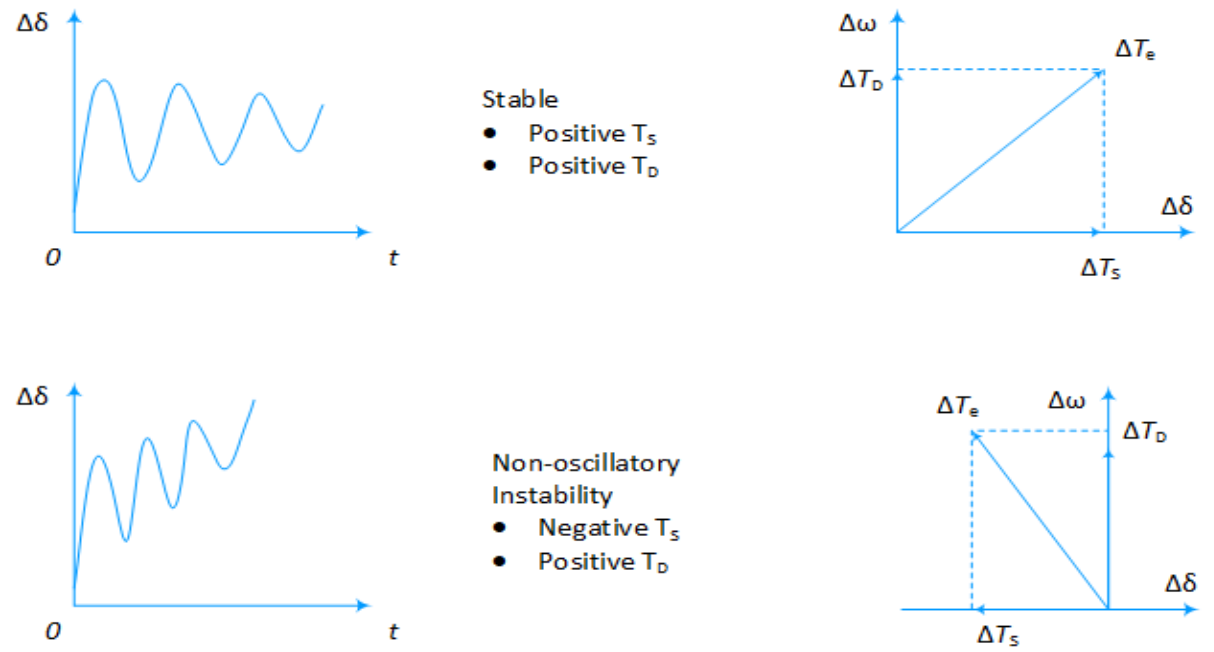

Non-oscillatory Instability

- Negative $T_{5}$

- Positive $T_{D}$

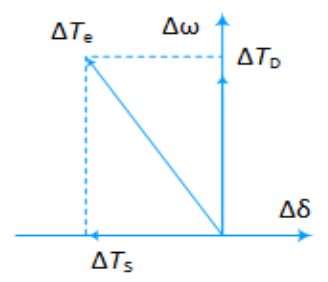

a) With constant field voltage

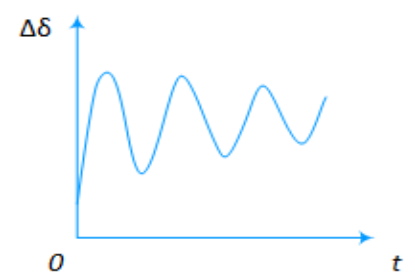

Stable

- Positive $T_{5}$

- Positive $T_{\mathrm{D}}$

0
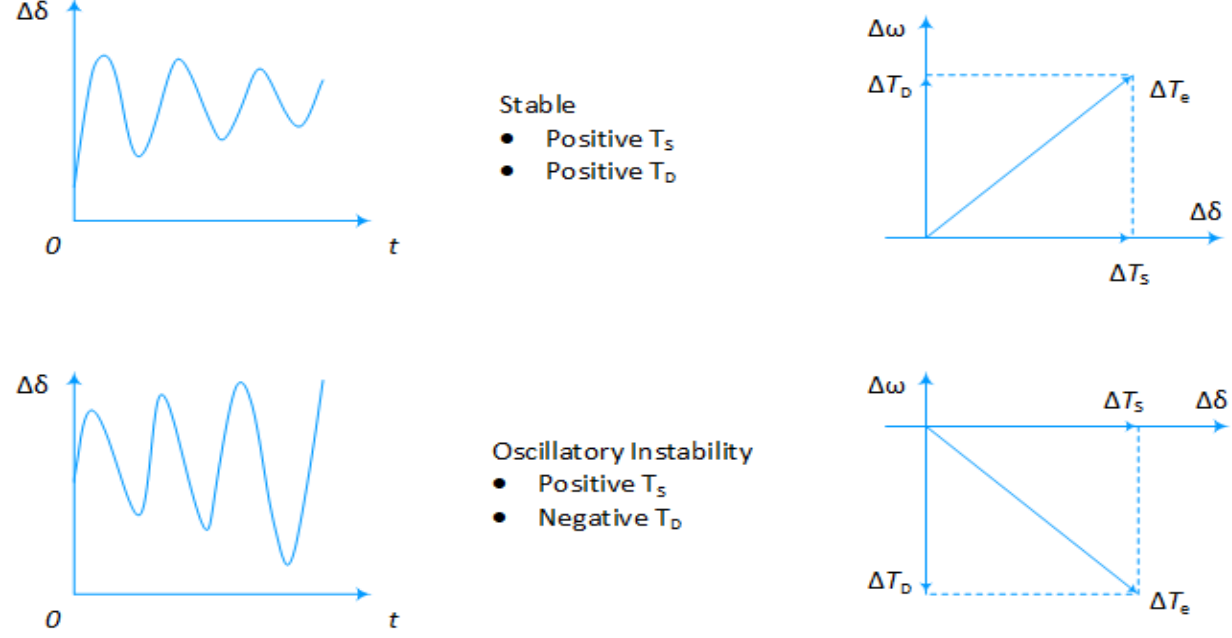

Oscillatory Instability

- Positive $T_{5}$

- Negative $T_{D}$

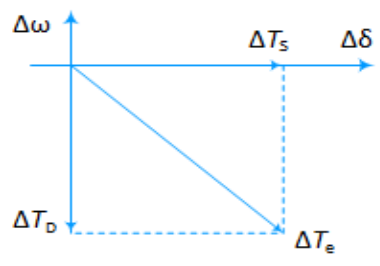

b) With excitation control

Figure 2.8: Nature of small-disturbance response [12]

Typical examples of small disturbances are a small change in the scheduled generation of one machine, which results in a change in its rotor angle $\delta$, or a small load added to the network.

The response of a power system to impacts is oscillatory. If the oscillations are damped and sufficient time has elapsed where the deviations in the state of the system are small, 
the system is stable. However, if the oscillations grow in magnitude or the oscillations are sustained indefinitely, the system is unstable.

If the power system is perturbed, it will acquire a new operating state. If the perturbation is small, the new operating state will not be significantly different from the initial one. Thus the operation is in the neighborhood of a certain quiescent $x_{0}$. In this limited range of operation a nonlinear system can be described mathematically by linearized equations.

The method of analysis used to linearize the differential equations describing system behavior is to assume small changes in system quantities such as $\delta_{\Delta}, \nu_{\Delta}$, and $P_{\Delta}$ (change in angle, voltage and power respectively). Equations for these variables are found by making Taylor series expansion about $x_{0}$ and neglecting higher order terms [14]. The behavior or the motion of these changes is then examined. In examining the dynamic performance of the system, it is important to ascertain not only that growing oscillations do not result during normal operations, but also that the oscillatory response to small impacts is well damped.

When the disturbances causing system changes disappear, the motion of the system is then free. Stability is then assured if the system returns to its original state. Such behavior can be determined in a linear system by examining the characteristic equation of the system. If the mathematical description of the system is in state-space form, i.e., if the system is described by a set of first order differential equations, the free response of the system can be determined from the eigenvalues of the $A$ matrix, as seen in equation 2.78 .

$$
\dot{x}=A x+B u
$$


Where $x$ is the vector of state variables, $B$ is the input matrix, and $u$ is the vector of input controls. Eigenvalues for a given matrix $A$ can be evaluated as a solution of equation 2.79. $\lambda$ is a vector of eigenvalues and $I$ is an identity matrix of the same order of matrix $A$.

$$
|\lambda I-A|=0
$$

If the order of the state matrix $A$ is $n x n$ then there will be $n$ eigenvalues, which could be real or complex.

- (i) When the eigenvalues have negative real parts, the original system is asymptotically stable.

- (ii) When at least one of the eigenvalues has a positive real part, the original system is unstable.

- (iii) When the eigenvalues have real parts equal to zero, it is not possible on the basis of the first approximation to say anything in general.

The time dependent characteristic of a mode corresponding to an eigenvalue $\lambda_{i}$ is given by $e^{\lambda_{i}{ }^{t}}$. Therefore, the stability of a system is determined by the eigenvalues as follows:

- (a) A real eigenvalue corresponds to a non-oscillatory mode. A negative real eigenvalue represents a decaying mode. The larger its magnitude, the faster the decay. A positive real eigenvalue represents aperiodic instability. 
- (b) Complex eigenvalues occur in conjugate pairs, and each pair corresponds to an oscillatory mode.

The associated c's (scalar product representing the magnitude of excitation) and eigenvectors will have appropriate complex values so as to make the entries of $x(t)$ real at every instant of time. Where in equation 2.80

$$
(a+j b) e^{(\sigma-j \omega) t}+(a-j b) e^{(\sigma+j \omega) t}
$$

has the form

$$
e^{\sigma t} \sin (\omega t+\theta)
$$

which represents a damped sinusoid for negative $\sigma$.

The real component of the eigenvalues gives the damping, and the imaginary component gives the frequency of oscillation of increasing amplitude. A negative real part represents a damped oscillation whereas a positive real part represents oscillation of increasing amplitude. Thus, for a complex pair of eigenvalues:

$$
\lambda=\sigma \pm j \omega
$$

The frequency of oscillation in $\mathrm{Hz}$ is given by 2.84 .

$$
f=\frac{\omega}{2 \pi}
$$

This represents the actual or damped frequency. The damping ratio is given by equation

$$
\zeta=\frac{-\sigma}{\sqrt{\sigma^{2}+\omega^{2}}}
$$


The damping ratio $\zeta$ determines the rate of decay of the amplitude of the oscillation, and the nature of the system response. If $\zeta$ is greater than 1 , both eigenvalues are real and negative; if $\zeta$ is equal to 1 , both eigenvalues are equal to $-\omega_{n}$; and if $\zeta$ is less than 1 , eigenvalues are complex conjugates, as seen in equation 2.85 .

$$
\begin{array}{r}
\lambda=-\zeta \omega_{n} \pm j \omega_{n} \sqrt{1-\zeta^{2}} \\
=\sigma \pm j \omega
\end{array}
$$

The location of the eigenvalues in the complex plane with respect to $\zeta$ and $\omega_{n}$ is illustrated in Figure 2.9.

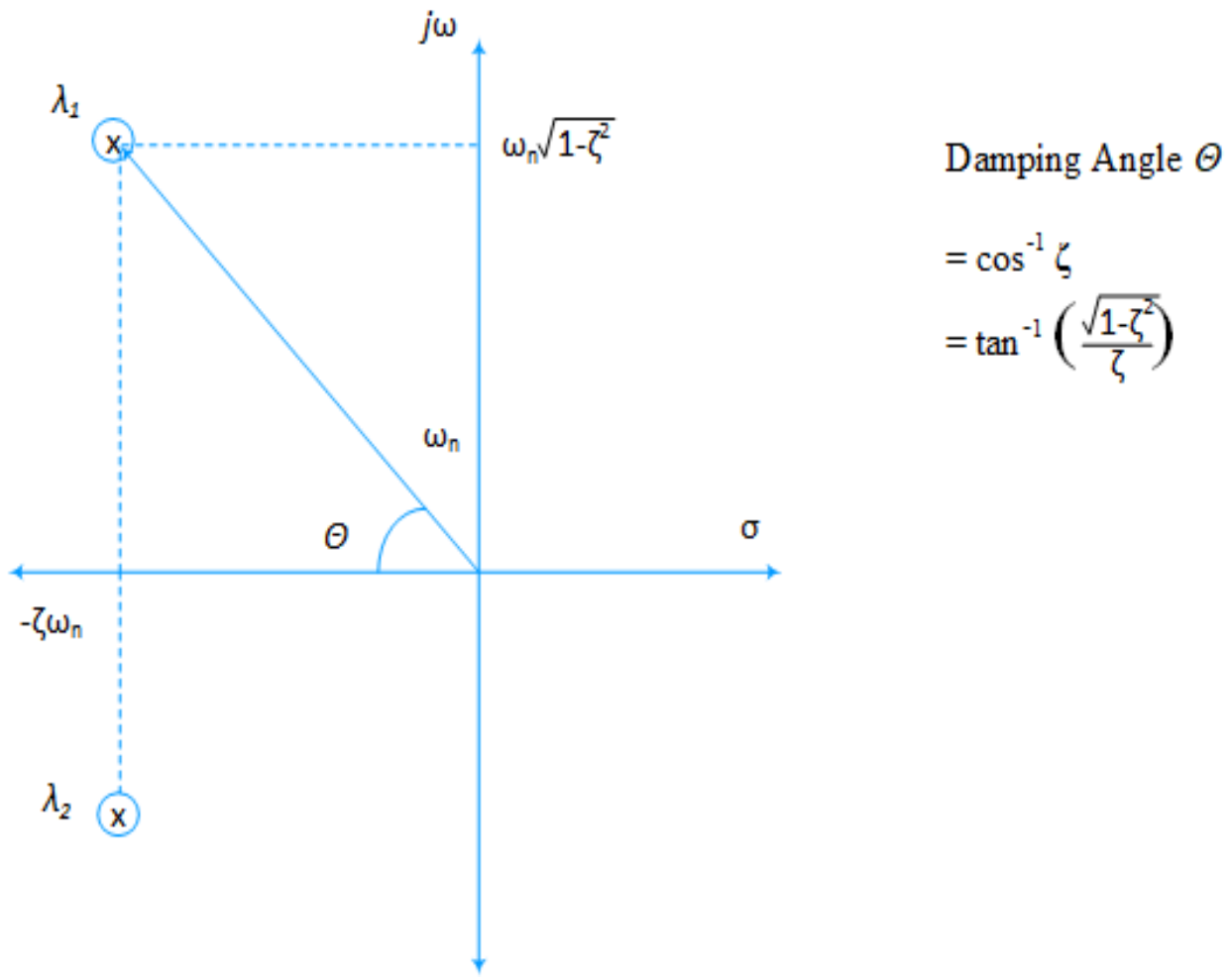

Figure 2.9: Eigenvalues damped cosine oscillations in complex plane [12] 


\subsubsection{Transient Stability}

The natural tendency of a generator connected to a power system is to deliver electrical power equal in amount to the mechanical power delivered to its shaft less its own losses. This condition of equilibrium is satisfied when the counter-torque due to armature currents and field is exactly equal to the mechanical torque applied at the shaft by the prime mover. When such a situation arises that there is a difference in the mechanical and electrical torque, the generator either speeds up or slows down until a new position of equilibrium is reached; and during the transition stage, the inertia forces due to the moving masses act in a manner which tends to prevent any change in speed [15].

The transient stability studies involve the determination of whether or not synchronism is maintained after the machine has been subjected to a severe transient disturbance. This may be sudden application or loss of load, or a fault on the system. The resulting response involves large excursions of generator rotor angles and is influenced by the nonlinear powerangle relationship. Stability depends on both the initial operating state of the system and the severity of the disturbance [12]. Usually, the system is altered so that the post disturbance steady-state operation differs from that prior to the disturbance.

The duration of a fault condition has a very important effect on the stability of a system. The fault condition reduces synchronising power directly by altering the equivalent circuit constants and indirectly by reducing the effective machine voltages through the demagnetizing action of fault currents [9]. 
In transient stability studies the period of interest is usually limited to 3 to 5 seconds following a disturbance, although it may extend to about ten seconds for very large systems with dominant interarea modes of oscillation [12].

\subsubsection{Damped Oscillations}

In describing transients and damped oscillation, it helps to consider a feature in forced oscillations, which is the energy in the oscillation. This is characterized in equation 2.86 .

$$
m \frac{d^{2} x}{d t^{2}}+\gamma m \frac{d x}{d t}+m \omega_{0}^{2} x=F(t)
$$

$F(t)$ is the cosine function on $t$. The work done by the force per second, i.e., the power, is the force times velocity. The differential work in a time $d t$ is $F d x / d t$, which is illustrated in equation 2.87. The Feynman Lectures on physics [16] provides an excellent discussion of units and dimensional analysis of damped oscillations.

$$
P=F \frac{d x}{d t}=m\left[\left(\frac{d x}{d t}\right)\left(\frac{d^{2} x}{d t^{2}}\right)+\omega_{0}^{2} x\left(\frac{d x}{d t}\right)\right]+\gamma m\left(\frac{d x}{d t}\right)^{2}
$$

The terms in brackets are the kinetic energy of motion and the other is the potential energy, or the energy stored in oscillation. When the average power is evaluated over many cycles when the oscillator is forced, all the energy ultimately ends up in the resistive term $\gamma m(d x / d t)^{2}$. The mean power $P$ is characterized by equation 2.88 .

$$
P=\gamma m\left(\frac{d x}{d t}\right)^{2}
$$


And the average power can be written as equation 2.89 .

$$
P=\frac{1}{2} \gamma m \omega^{2} x_{0}^{2}
$$

At any moment there is a certain amount stored energy $E$, which can be written as equation 2.90 .

$$
\begin{array}{r}
E=\frac{1}{2} m\left[\left(\frac{d x}{d t}\right)^{2}\right]+\frac{1}{2} m \omega_{0}^{2}\left(x^{2}\right) \\
E=\frac{1}{2} m\left(\omega^{2}+\omega_{0}^{2}\right) \frac{1}{2} x_{0}^{2}
\end{array}
$$

Stored energy is called the $Q$ of the system, and $Q$ is defined as $2 \pi$ times the mean stored energy, divided by the work done per cycle as illustrated in equation 2.91 .

$$
Q=2 \pi \frac{\frac{1}{2} m\left(\omega^{2}+\omega_{0}^{2}\right)\left(x^{2}\right)}{\gamma m \omega^{2}\left(x^{2}\right) \frac{2 \pi}{\omega}}=\frac{\omega^{2}+\omega_{0}^{2}}{2 \gamma \omega}
$$

For a good oscillator close to resonance, equation 2.91 can be simplified by setting $\omega=\omega_{0}$, where equation 2.92 is the definition of $Q$.

$$
Q=\frac{\omega_{n}}{\gamma}
$$

Where $L$ can be substituted for $m, R$ for $m \gamma$, and $\frac{1}{C}$ for $m \omega_{0}^{2}$. The $Q$ at resonance is $\frac{L \omega}{R}$, where $\omega$ is the resonance frequency, and $\gamma=\frac{R}{L}$ is the electrical resistance. A transient is a solution of the differential equation where there is no force present, but the system is simply not at rest. Suppose an oscillation is driven by a force for awhile, then the force is removed for a very high $Q$ system. When the force is acting, the stored energy stays the same, and there is a certain amount of work done to maintain it. When the force is removed, and no more work is being done; then the losses consume the energy of the supply until there is 
no more driver. The losses will have to consume the energy that is stored. For example, if $\frac{Q}{2 \pi}=1000$. Then the work done per cycle is $\frac{1}{1000}$ of the stored energy. Since it is oscillating with no driving force, that in one cycle the system will still lose a thousandth of its energy $E$, which would normally have been supplied by the driver, and it will continue oscillating, always losing $\frac{1}{1000}$ of its energy per cycle.

For a high $Q$ system, equation 2.93 would apply. In each radian the system losses a fraction $\frac{1}{Q}$ stored energy $E$.

$$
\frac{d E}{d t}=-\omega \frac{E}{Q}
$$

Thus in a given amount of time $d t$ the energy will change by an amount $\omega \frac{d t}{Q}$, since the number of radians associated with the time $d t$ is $\omega d t$. With respect to its frequency, the system moves with little force, so it will oscillate at essentially the same frequency. Where $\omega$ is the resonant frequency $\omega_{0}$, and the stored energy is characterized by equation 2.94 . This would be the measure of the energy at any moment.

$$
E=E_{0} e^{-\omega_{0} \frac{t}{Q}}=E_{0} e^{-\gamma t}
$$

With respect to the amplitude of the oscillation of time, the energy in a spring goes as the square of velocity; so the total energy goes as the square of displacement. Thus the displacement, the amplitude of oscillation, will decrease half as fast because of the square. The damped transient motion will be an oscillation of frequency close to the resonance frequency $\omega_{0}$, in which case the amplitude of the sine-wave motion will diminish as $e^{-\gamma \frac{t}{2}}$ 
This is characterized by equation 2.95, and is illustrated in Figure 2.10.

$$
x=A_{0} e^{-\gamma \frac{t}{2}} \cos \omega_{0} t
$$

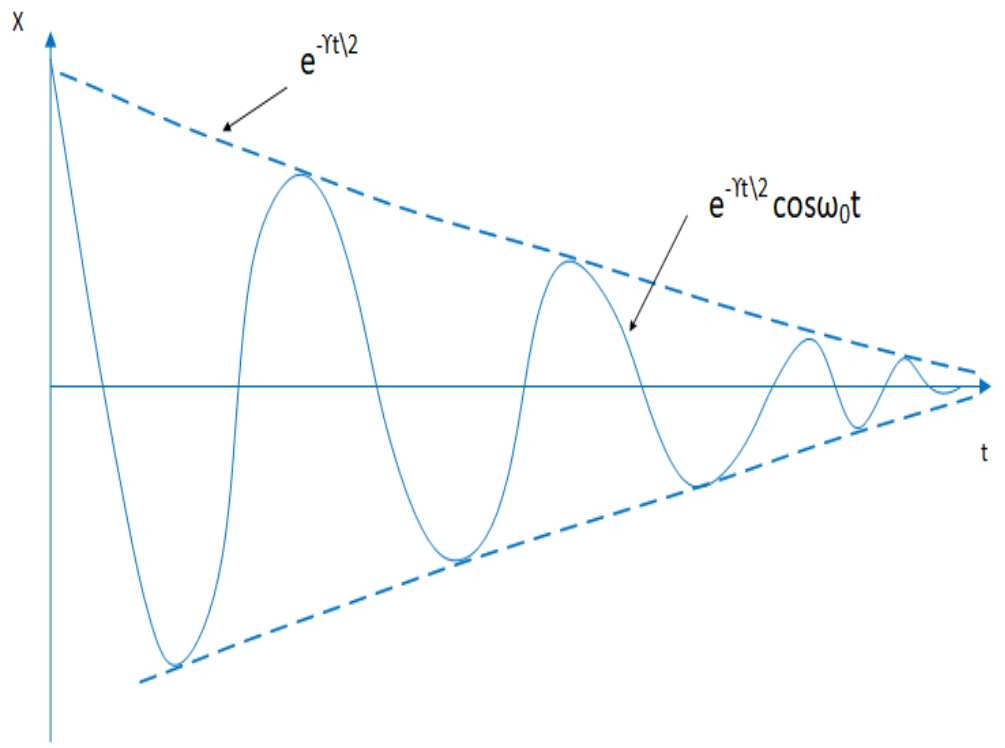

Figure 2.10: Damped Cosine Oscillation [16]

To analyze the differential equation of the motion itself, it can be evaluated as a solution of an exponential curve, $x=A e^{i \alpha t}$. Evaluating this term in equation 2.86 with $F(t)=0$, using the rule that each time you differentiate with $x$ with respect to time, it is multiplied by $i \alpha$. After substitution equation 2.96 is obtained.

$$
\left(-\alpha^{2}+i \gamma \alpha+\omega_{0}^{2}\right) A e^{i \alpha t}=0
$$

The net result be zero at all times, which is not possible unless $A=0$, where it would motionless. If the equation was evaluated as equation 2.97 ,

$$
-\alpha^{2}+i \gamma \alpha+\omega_{0}^{2}=0
$$


this can be solved to find $\alpha$ by equation 2.98 , which will provide a solution of $A$ that will not be zero.

$$
\alpha=i \frac{\gamma}{2} \pm \sqrt{\omega_{0}^{2}-\frac{\gamma^{2}}{4}}
$$

Where $\gamma$ is small compared with $\omega_{0}$, so that $\omega_{0}^{2}-\frac{\gamma^{2}}{4}$ is positive. This provides two solutions seen in equations 2.99 and 2.100 .

$$
\begin{aligned}
& \alpha_{1}=i \frac{\gamma}{2} \pm \sqrt{\omega_{0}^{2}-\frac{\gamma^{2}}{4}}=i \frac{\gamma}{2}+\omega_{\gamma} \\
& \alpha_{2}=i \frac{\gamma}{2} \pm \sqrt{\omega_{0}^{2}-\frac{\gamma^{2}}{4}}=i \frac{\gamma}{2}-\omega_{\gamma}
\end{aligned}
$$

The solution for $x$ is $x_{1}=A e^{i \alpha_{1} t}$, where $A$ is a constant. By substituting $\alpha_{1}$ in the first part of the two solutions, and calling $\sqrt{\omega_{0}^{2}-\frac{\gamma^{2}}{4}}=\omega_{\gamma}$. Thus $i \alpha_{1}=-\frac{\gamma}{2}+i \omega_{\gamma}$, and we obtain $x=A e^{\left(-\frac{\gamma}{2}+i \omega_{\gamma}\right) t}$, which is characterized in equation 2.101 .

$$
x_{1}=A e^{-\gamma \frac{t}{2}} e^{i \omega_{\gamma} t}
$$

This is an oscillation at frequency $\omega_{\gamma}$, which is close to frequency $\omega_{0}$. The amplitude of oscillation is decreasing exponentially, and by taking the real part in equation 2.101 , we get equation 2.102. This is similar to equation 2.95, except with a frequency $\omega_{\gamma}$.

$$
x_{1}=A e^{-\gamma \frac{t}{2}} \cos \omega_{\gamma} t
$$

The other solution is $\alpha_{2}$, is seen in equation 2.103 when $\omega_{\gamma}$ is reversed and set negative.

$$
x_{2}=B e^{-\gamma \frac{t}{2}} e^{-i \omega \gamma t}
$$

This will illustrate that $x_{1}$ and $x_{2}$ are a solution of equation equation 2.95 with $F=0$, then $x_{1}+x_{2}$ is also a solution. so the general solution for $x$ is characterized in equation 2.104

$$
x=e^{-\gamma \frac{t}{2}}\left(A e^{i \omega \gamma t}+B e^{i \omega \gamma t}\right)
$$


For $x$ to be real, $B e^{-\omega_{\gamma} t}$ will have to be the complex conjugate of $A e^{\omega_{\gamma} t}$ that the imaginary parts will disappear. This shows that $B$ is the complex conjugate of $A$ so the real solution $x$ is seen in equation 2.105. This is an oscillation with a phase shift and a damping.

$$
x=e^{-\gamma \frac{t}{2}}\left(A e^{i \omega \gamma t}+A^{*} e^{i \omega \gamma t}\right)
$$

\subsubsection{Characteristic Equations}

The natural frequencies of oscillation of a synchronous machine, when perturbed, will have several modes of oscillations with respect to the rest of the system. There are cases where coherent groups of machines oscillate with respect to other groups of machines. The oscillations cause fluctuations in bus voltages, system frequencies, and tie-line power flows.

When there is a difference in angular velocity between rotor and air gap, and induction torque will be set up on the rotor tending to minimize the difference of velocities.

Referencing the linear second-order differential equation from 2.50 , which is written in the form of the following

$$
\frac{d^{2} \delta_{\Delta}}{d t^{2}}+\frac{\omega_{s} S_{p}}{2 H} \delta_{\Delta}=0
$$

Introduce the damping power into swing equation

$$
P_{d}=D \frac{d \delta}{d t}
$$

Lets substitute $P_{s}$ for $S_{p}$ from equation 2.49 and as seen in equation 2.50 for the synchronizing power coefficient. This will be used for the following characteristic equations: 
Solution of Swing Equation

$$
\begin{aligned}
& \frac{H}{\pi f_{0}} \frac{d^{2} \Delta \delta}{d t^{2}}+D \frac{d \Delta \delta}{d t}+P_{s} \Delta \delta=0 \\
& \frac{d^{2} \Delta \delta}{d t^{2}}+2 \zeta \omega_{n} \frac{d \Delta \delta}{d t}+\omega_{n}^{2} \Delta \delta=0
\end{aligned}
$$

Dividing the left-hand side of equation 2.107 by $P_{s}$ will result in equation 2.109 .

$$
\frac{\frac{H}{P_{S}}}{\pi f_{0}} \frac{d^{2} \Delta \delta}{d t^{2}}+\frac{D}{P_{s}}+\Delta \delta=0
$$

Multiplying the left-hand side of equation 2.108 by $\frac{1}{\omega_{n}^{2}}$ will result in equation 2.110 .

$$
\frac{1}{\omega_{n}^{2}} \frac{d^{2} \Delta \delta}{d t^{2}}+\frac{2 \zeta \omega_{n}}{\omega_{n}^{2}}+\Delta \delta=0
$$

Further reducing, the left-hand side of equation 2.109 can be rewritten as equation 2.111 .

$$
\left\langle\frac{D}{P_{s}}=\frac{2 \zeta}{\omega_{n}}\right\rangle
$$

Then the left-hand side of equation 2.110 can be rewritten as equation 2.112 .

$$
\left\langle\frac{H}{P_{s}}=\frac{\pi f_{0}}{\omega_{n}^{2}}\right\rangle
$$

Applying Laplace transformation to equation 2.107 we obtain equation 2.113 .

$$
s^{2}+\frac{\pi f_{s}}{H} D_{s}+\frac{\pi f_{s}}{H} P_{s}=0
$$

As seen in equation 2.113, this is characteristic of the standard second-order system written in equation 2.114 .

$$
s^{2}+2 \zeta \omega_{n} s+\omega_{n}^{2}=0
$$


Additionally, we can characterize equations 2.115 and 2.116 from the second-order equation.

The Natural Frequency

$$
\omega_{n}=\sqrt{\frac{\pi f_{s}}{H} P_{s}}
$$

The Damping ratio

$$
\zeta=\frac{D}{2} \sqrt{\frac{\pi f_{s}}{H P_{s}}}
$$

The eigenvalues in equation 2.113 can also be evaluated from the roots of characteristic equation given in equation 2.114 to obtain equation 2.117 .

$$
s_{1}, s_{2}=-\zeta \omega_{n} \pm j \omega_{n} \sqrt{1-\zeta^{2}}
$$

Damped frequency of oscillation.

$$
\omega_{d}=\omega_{n} \sqrt{1-\zeta^{2}}
$$

Roots of Swing Equation

$$
\begin{gathered}
\Delta \delta=\frac{\Delta \delta}{\sqrt{1-\zeta^{2}}} e^{-\zeta \omega_{n} t} \sin \left(\omega_{d} t+\theta\right) \\
\delta=\delta_{0}+\frac{\Delta \delta}{\sqrt{1-\zeta^{2}}} e^{-\zeta \omega_{n} t} \sin \left(\omega_{d} t+\theta\right)
\end{gathered}
$$

Rotor Angel Frequency

$$
\Delta \omega=-\frac{\omega_{n} \Delta \delta}{\sqrt{1-\zeta^{2}}} e^{-\zeta \omega_{n} t} \sin \left(\omega_{d} t\right)
$$




$$
\omega=\omega_{0}+\frac{\omega_{n} \Delta \delta}{\sqrt{1-\zeta^{2}}} e^{-\zeta \omega_{n} t} \sin \left(\omega_{d} t\right)
$$

Response time constant

$$
\tau=\frac{1}{\zeta \omega_{n}}=\frac{2 H}{\pi f_{0} D}
$$

Settling time

$$
t_{s} \cong 4 \tau
$$

As $H$ increases $t_{s}$ will increase, while $\omega_{n}$ and $\zeta$ decrease.

\subsection{Numerical Solutions of the Swing Equation}

Transient stability analysis requires the solution of a system of coupled non-linear differential equations by numerical integration, and direct methods.

Any one of several well known step-by-step procedures may be chosen for numerical integration of the differential equations. The fourth-order Runge-Kutta method is often used in production-type transient stability programs. Other methods, such as the Euler method, the Modified Euler method, the trapezoidal method, and predictor-corrector methods like the following step-by-step method are alternatives. Since classical stability studies have been performed for many years, long before digital computers became available, it is worthwhile to look at the techniques which were used for hand calculations [17]. 


\subsubsection{Step-by-Step Method}

For large systems we depend on the digital computer which determines $\delta$ versus $t$ for all machines in which we are interested; and $\delta$ may be plotted versus $t$ for a machine to determine the swing curve of that machine. The angle $\delta$ is calculated as a function of time over a period long enough to determine whether $\delta$ will increase without limit or reach a maximum and start to decrease. Although the latter result usually indicates stability, on an actual system where a number of variables are taken into account it may be necessary to plot $\delta$ versus $t$ over a long enough interval to be sure $\delta$ will not rise again without returning to a low value. Stevenson [4] and Kimbark [2] provide an excellent discussion of units and dimensional analysis of step-by-step solution to the swing equation.

By determining swing curves for various clearing times the length of time permitted before clearing a fault can be determined. Standard interrupting times after a fault for circuit breakers and their associated relays are commonly 8, 5, 3 or 2 cycles after a fault occurs and thus breaker speeds may be specified. Calculations should be made for a fault in the position which will allow the least transfer of power from the machine and for the most severe type of fault for which protection against loss of stability is justified.

A number of different methods are available for the numerical evaluation of second-order differential equations in step-by-step computations for small increments of the independent variable. The more elaborate are only practical when the computations are performed on a digital computer. The step-by-step method used for hand calculation is necessarily simpler. 
In the method for hand calculation the change in the angular position of the rotor during a short interval time is computed by making the following assumptions:

1. The accelerating power $P_{a}$ computed at the beginning of an interval is constant from the middle of the preceding interval to the middle of the interval considered.

2. The angular velocity is constant throughout any interval at the value computed for the middle of the interval. Of course, neither of the assumptions are true, since $\delta$ is changing continuously and both $P_{a}$ and $\omega$ are functions of $\delta$. As the time interval is decreased, the computed swing curve approaches the true curve.

Figure 2.11 will help in visualizing the assumptions. the accelerating power is computed for the points enclosed in circles at the ends of the $n-2, n-1$, and $n$ intervals, which are the beginning of the $n-1, n$, and $n+1$ intervals. The step curve of $P_{a}$ in Figure 2.11 results from the assumption $P_{a}$ is constant between midpoints of the intervals. Similarly, $\omega_{r}$, the excess of the angular velocity $\omega$ over the synchronous angular velocity $\omega_{s}$, is shown as a step curve that is constant throughout the interval at the value computed for the midpoint. Between the coordinates $n-\frac{3}{2}$ and $n-\frac{1}{2}$ there is a change in speed caused by the constant accelerating power. The change in speed is the product of the acceleration and the time interval, and as seen in equation 2.125

$$
\omega_{r, n-\frac{1}{2}}-\omega_{r, n-\frac{3}{2}}=\frac{d^{2} \delta}{d t^{2}} \Delta t=\frac{180 f}{H} P_{a, n-1} \Delta t
$$




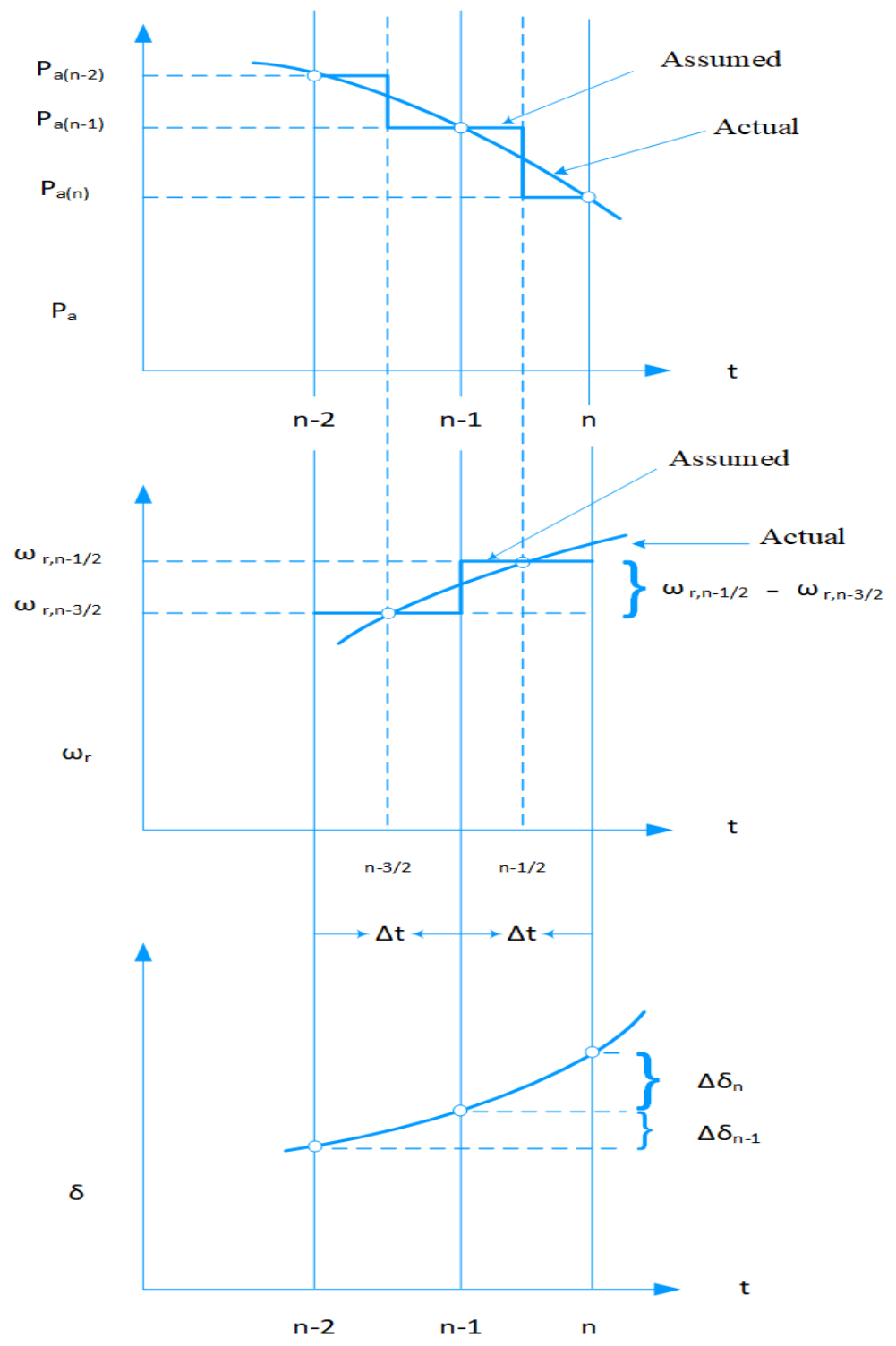

Figure 2.11: Actual and assumed values of $P_{a}, \omega_{r}$, and $\delta$ as functions of time [4]

The change in $\delta$ over any interval is the product of $\omega_{r}$ for the interval and the time interval.

Thus, the change in $\delta$ during the $n-1$ interval is written in equation 2.126 .

$$
\Delta \delta_{n-1}=\delta_{n-1}-\delta_{n-2}=\Delta t \omega_{r, n-\frac{3}{2}}
$$

and during the nth interval written in equation 2.127

$$
\Delta \delta_{n}=\delta_{n}-\delta_{n-1}=\Delta t \omega_{r, n-\frac{1}{2}}
$$


Subtracting equation 2.126 from equation 2.127 an substituting equation 2.125 in the resulting equation to eliminate all values of $\omega_{r}$ yields equation 2.128.

$$
\Delta \delta_{n}=\Delta \delta_{n-1}+k P_{a, n-1}
$$

where equation 2.129 is used.

$$
k=\frac{180 f}{H}(\Delta t)^{2}
$$

Equation 2.128 is the important one for the step-by-step solution of the swing equation with the necessary assumptions enumerated, for it shows how to calculate the change in $\delta$ during an interval if the change in $\delta$ for the previous interval and the accelerating power for the interval in question are known. Equation 2.128 shows that, subject to the stated assumptions, the change in torque angle during a given interval is equal to the change in torque angle during the proceeding interval plus the accelerating power at the beginning of the interval times $k$. The solution progresses through enough intervals to obtain points for plotting the swing curve. Greater accuracy is obtained when the duration of the intervals is small. An interval of $0.05 s$ is usually satisfactory.

The occurrence of a fault cause a discontinuity in the accelerating power power $P_{a}$, which is zero before a fault and a definite amount immediately following the fault. The discontinuity occurs at the beginning of the interval, when $t=0$. Reference to Figure 2.11 shows that the method of calculation assumes that the acceleration power computed at the beginning of an interval is constant from the middle of the proceeding interval to the middle of the interval considered. When the fault occurs, we have two values of $P_{a}$ at the beginning 
of an interval, and we must take the average of those two values as our constant accelerating power.

There are two factors which can act as guideline criteria for the relative stability of a generating unit within a power system. These are the angular swing of the machine during and following a fault conditions and the critical clearing times. The $H$ constant and the transient reactance $X_{d}^{\prime}$ of the generating unit have a direct effect on both these criteria.

Evaluation of equation 2.128 and equation 2.129 indicates that the smaller the $H$ constant, the larger the angular swing during any time interval. On the other hand, equation 2.36 shows that $P_{\max }$ decreases as the transient reactance of the machine increases. This is so because the transient reactance forms part of the overall series reactance of the system which is the reciprocal of the transfer admittance. Examination of Figure 2.11 shows that all three power curves are lowered when $P_{\max }$ is decreased. Accordingly, for a given shaft power $P_{m}$, the initial rotor angle $\delta_{0}$ is increased, $\delta_{\max }$ is decreased, and a smaller difference between $\delta_{0}$ and $\delta_{c r}$ exists for a smaller $\delta_{\max }$. The net result is a decreased $P_{\max }$ constrains a machine to swing through a smaller angle from its original position before it reaches a critical clearing time to decrease and lessons the probability of maintaining stability under transient conditions. 


\subsubsection{Euler Method}

The differential equations to be solved in power system stability analysis are nonlinear ordinary differential equations with known initial values:

$$
\frac{d x}{d t}=f(x, t)
$$

as illustrated in equation 2.130, where $x$ is the state vector of $n$ dependent variables and $t$ is the independent variable time. The objective is to solve $x$ as a function of $t$, with the initial values of $x$ and $t$ equal to $x_{0}$ and $t_{0}$, respectively. Kundur [12] provides an excellent discussion of units and dimensional analysis of numerical integration techniques of Euler and Runge-Kutta (R-K) methods.

Evaluating the first-order differential equation 2.130 with $x=x_{0}$ at $t=t_{0}$. Figure 2.12 illustrates the principle of applying the Euler method. At $x=x_{0}, t=t_{0}$ we can approximate the curve representing the true solution by its tangent having a slope illustrated below

$$
\left.\frac{d x}{d t}\right|_{x=x_{0}}=f\left(x_{0}, t_{0}\right)
$$

where $\Delta_{x}$,

$$
\Delta_{x}=\left.\frac{d x}{d t}\right|_{x=x_{0}} \cdot \Delta t
$$

The value of $x$ at $t=t_{1}=t_{0}+\Delta t$ is given by

$$
x_{1}=x_{0}+\Delta x=x_{0}+\left.\frac{d x}{d t}\right|_{x=x_{0}} \cdot \Delta t
$$


The Euler method is equivalent to using the first two terms of the Taylor series expansion or $x$ around the point $\left(x_{0}, t_{0}\right)$ as seen in equation 2.132 .

$$
x_{1}=x_{0}+\Delta(\dot{x})+\frac{\Delta^{2}}{2 !}\left(\ddot{x}_{0}\right)+\frac{\Delta t^{3}}{3 !}\left(\dddot{x}_{0}\right)+\cdots
$$

After using Euler technique for determining $x=x_{1}$ corresponding to $t=t_{1}$ then another short time step $\Delta t$ is taken to determine $x_{2}$ corresponding to $t_{2}=t_{1}+\Delta t$ as seen in equation

$$
x_{2}=x_{1}+\left.\frac{d x}{d t}\right|_{x=x_{1}} \cdot \Delta t
$$

By applying the technique successfully, values of $x$ can be determined corresponding to different vales of $t$.

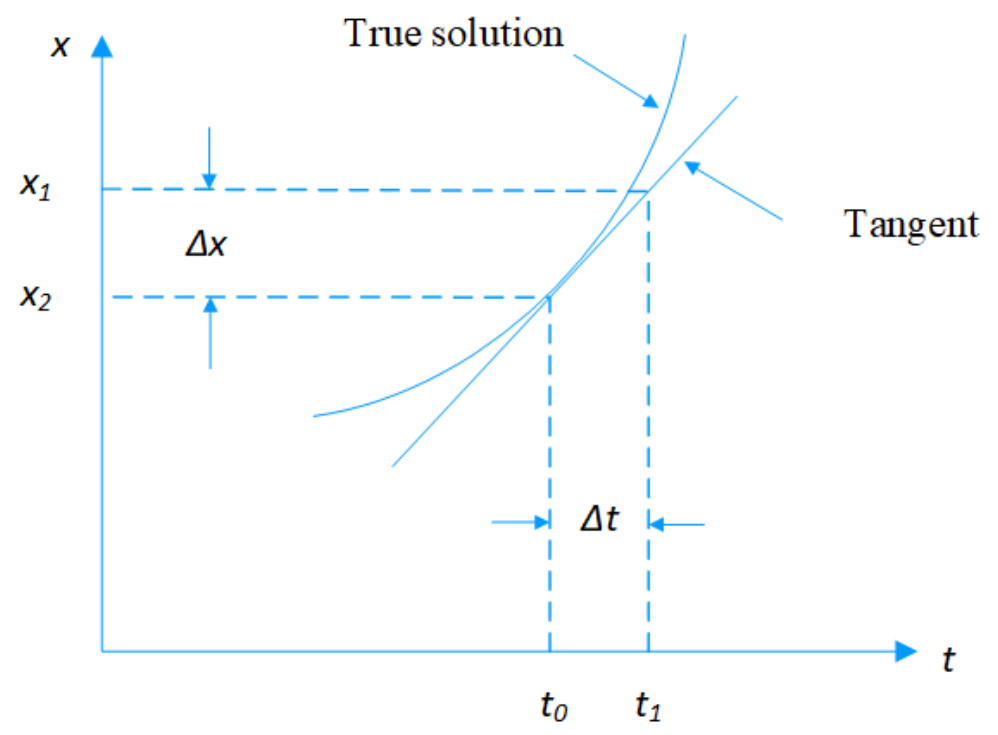

Figure 2.12: Euler Method [12]

The method considers only the first derivative of $x$ and is referred to as a first-order method.To give sufficient accuracy for each step, $\delta t$ has to be small. This will increase round-off errors, and the computational effort will be very high. 
In the application of numerical integration methods, it is important to consider the propagation of error, which may cause slight errors made early in the process to be magnified in later steps. Numerical stability depends on the propagation of error. If early errors carry through but cause no significant further errors later, the method is said to be numerically stable. If early errors cause other large errors later, the method is said to be numerically unstable.

\subsubsection{Modified Euler Method}

The standard Euler method results in inaccuracies because it uses the derivative at the beginning of the interval as though it applied throughout the interval. The modified Euler method tries to overcome this problem by using the average of the derivatives at the two ends. The modified Euler consists of the following steps: predictor step. By using the derivative at the beginning of the step, the value at the end of the step is predicted as seen in equation 2.134 .

$$
x_{1}^{p}=x_{0}+\left.\frac{d x}{d t}\right|_{x=x_{0}} \cdot \Delta t
$$

Corrector step. By using the predicted value of $x_{1}^{p}$, the derivative at the end of the step is computed and the average of this derivative and the derivative at the beginning of the step is used to find the corrected value as written in equation 2.135 .

$$
x_{1}^{c}=x_{0}+\frac{1}{2}\left(\left.\frac{d x}{d t}\right|_{x=x_{0}}+\left.\frac{d x}{d t}\right|_{x=x_{1}^{p}}\right) \Delta t
$$

If desired, a more accurate value of the derivative at the end of the step can be calculated, again by using $x=x_{1}^{c}$. This derivative can be used to calculate a more accurate value of the 
average derivative, which is used to apply the corrector step again. This process can be used repeatedly until successive steps converge with the desired accuracy.

\subsubsection{Runge-Kutta (R-K) Methods}

The R-K methods approximate the Taylor series solution. However, unlike the formal Taylor series solution, the R-K methods do not require explicit evaluation of the derivatives higher than the first. The effects of the higher derivatives are included by several evaluations of the first derivative. Depending on he number of terms effectively retained in the Taylor series, there are R-K methods of different orders.

\subsubsection{Second-Order (R-K) Method}

Referring to the differential equation 2.130, the second-order R-K formula for the value of $x$ at $t=t_{0}+\Delta t$ is written in equation 2.136

$$
x_{1}=x_{0}+\Delta x=x_{0}+\frac{k_{1}+k_{2}}{2}
$$

Where

$$
\begin{gathered}
k_{1}=f\left(x_{n}, t_{n}\right) \Delta t \\
k_{2}=f\left(x_{n}+k_{1}, t_{n}+\Delta t\right) \Delta t
\end{gathered}
$$

\subsubsection{Fourth-Order (R-K) Method}

The general formula giving the value of $x$ for the $(n+1)^{\text {st }}$ step is written in equation 2.137.

$$
x_{n+1}=x_{n}+\frac{1}{6}\left(k_{1}+2 K_{2}+2 k_{3}+k_{4}\right)
$$


Where

$$
\begin{gathered}
k_{1}=f\left(x_{n}, t_{n}\right) \Delta t \\
k_{2}=f\left(x_{n}+\frac{k_{1}}{2}, t_{n}+\frac{\Delta t}{2}\right) \Delta t \\
k_{3}=f\left(x_{n}+\frac{k_{1}}{2}, t_{n}+\frac{\Delta t}{2}\right) \Delta t \\
k_{4}=f\left(x_{n}+k_{3}, t_{n}+\Delta t\right) \Delta t
\end{gathered}
$$

The physical interpretation of the above solution is as follows:

- $k_{1}=($ slope at the beginning of the time step $) \Delta t$

- $k_{2}=($ first approximation to the slope at mid-step $) \Delta t$

- $k_{3}=($ second approximation to the slope at mid-step $) \Delta t$

- $k_{4}=($ slope at the end of step) $\Delta t$

- $\Delta x=\frac{1}{6}\left(k_{1}+2 k_{2}+2 k_{3}+k_{4}\right)$

Where $\Delta x$ is the incremental value of $x$ given by the weighted average of estimates based on slopes at the beginning, midpoint, and end of the time step.

\subsection{How do D \& H characterize the system}

In the initial form of the swing equation 2.8, as written below, the damping term (proportional

to $\frac{d \delta}{d t}$ ) is absent because the assumption of a lossless machine and the torque damper winding 
had been ignored.

$$
M \frac{d^{2} \delta}{d t^{2}}=P_{s}=P_{m}-P_{e}
$$

Damping needs to be considered in transient analysis since $P_{e}$ depends upon the sine angle of $\delta$, as seen in the power angle equation 2.37 .

The inertia constant $H$ and damping coefficient $D$ in our original swing equation can be illustrated in a synchronous machine in characteristic equation 2.107.

$$
\frac{H}{\pi f} \frac{d^{2} \delta}{d t^{2}}+D \frac{d \delta}{d t}=P_{s}=P_{m}-P_{e}
$$

Maintaining electrical grid frequency within an acceptable range is critical for stability to a power system that constantly experiences fluctuations in generation and demand. One of the primary requirements for reliable operation of a power system is dependent on a stable frequency response.

This frequency response, or rate at which grid frequency changes is referred to as the Rate of Change of Frequency (ROCOF). Frequency response is dependent on the inertia constant $H$, and damping coefficient $D$, both of which are critical system parameters that characterize the power system. The inertia constant and damping coefficient are both frequency dependent. Typical values for $H$ range from 2-10 seconds [11]. Where as the damping constant, a typical estimate is that a $1 \%$ decline in frequency will reduce load by $1 \%-2 \%$, which is a damping constant between 1-2 [18]. 
Inertia in a power system refers to the energy stored in synchronous generators and some large industrial motors. The actual relationship between energy and frequency can be referenced to equation 2.90, and is given by the equation $E=I \omega^{2}$ where $I$ is the generators moment of inertia (a quantity determined by the amount and distribution of mass of the generator), and $\omega$ is the rotational speed of the system. This stored kinetic energy in the form of rotational inertia provides a tendency to remain rotating, which is an important property in maintaining a stable frequency. If a power source fails, inertia resists the drop in frequency, which provides the grid time to balance generation and demand [18]. This is due to the electro-mechanical behavior, a generator's rotating mass provides kinetic energy to the grid, or absorbs energy from the grid, in case of frequency deviation[12]. The combination of inertia constant and total capacity of online synchronous generators determines the total inertia. Grid size is a key factor in the total inertia, and therefore how fast the frequency declines.

Damping coefficient represents a variety of damping sources, including control loops and loads. The actual damping constant for loads in the United States is not well understood. [18].

Analysis of damping can be complex, but yields a physical explanation for positive and negative damping torques of a synchronous machine. It has been found that the torque of a synchronous machine consists of three components, two dependent on the speed of the machine and the third on the angle between the rotating field and the rotor. When a synchronous machine oscillates, the change of the first two components produces 
positive and negative damping torques of the synchronous machine. The change in the third component produces synchronizing torque. The positive component of the damping torque is called rotor-damping while the negative component is called stator-damping.[19]. The damping coefficient $\mathrm{D}$ includes the various damping torque components, both mechanical and electrical. Values of the damping coefficient usually used in stability studies are in the range of 1-3 pu [2].

It is recognized that synchronous machines connected to a power system exhibit electrical torques giving a spring action (torques proportional to angular displacement) and damping action (torques proportional to velocity). The spring action together with inertia results in a torsional natural frequency of the order of 1-to-4 cycles per second [20].

The damping coefficient is the inverse of the time constant of the system. The significance of which, determines the rate at which the change in frequency returns to an acceptable range after a disturbance, or event that causes a deviation in frequency.

\subsection{How, historically, have $\mathrm{D} \& \mathrm{H}$ been determined}

Historically, since the early work of Bell and Louis's Practical Properties of Polyphase Apparatus in 1894 to C. P. Steinmetz's Notes on the theory of Synchronous Motors in 1902, a great attention and effort was focused to studying the problem of stability between 1919-1934 [9].

The synchronous machine formulas are based on the vector diagrams developed by R. E. 
Doherty and C. A. Nickle in their series of of papers in 1926 [21] on synchronous machines, and also developed by R. H Park in his paper on Two-reaction Theory 1929 [22]. Equations for the damping and synchronizing components of pulsating torque caused by a given small angular position of the rotor circuits had been derived by Park. These papers also provided early details of the step-by-step stability calculations as noted by F. R. Longley in 1930 [23].

Equations for damping torques angles of synchronous machines during disturbances were further developed by S. B. Crary and M. L. Waring in 1932 [24], which was essentially based on the numerical evaluation of Duhamel Integrals .

As noted by M. M. Liwschitz in 1941, the straight mathematical analysis of the damping problem on the synchronous machine leads to very complicated results. From these results, the conception of the phenomena occurring in the machine is very difficult, and in addition the formulas obtained are so long that the calculation becomes tedious and errors could be easily made [19].

Descriptions of equivalent circuits were developed that were preferable to calculating synchronizing torques as though the field was the only circuit in the rotor, and the amortisseur or additional circuits regarded simply as that of producing damping torque is equal to $T_{d} p \delta$ [22]. Where $T_{d}$ represents the damping torque coefficient for small oscillations about the angle $\delta$.

Mathematical analysis on the damping problem had progressed in developing less complicated physical explanations for the positive and negative damping of synchronous 
machines [19], on the basis to derive simpler formulas for the calculation of damping torques; positive component of the rotor-damping and the negative component of the stator-damping. Where rotor-damping is is determined directly by the slope of the speed-torque curves. Approximate formula values for stator-damping could be determined.

Further developments on simplifying formulas for spring and damping coefficients of synchronous machines were created by L. A. Kilgore and E. C. Whitney 1950 [20], where they developed a more exact method. For very low frequency oscillations (less than one cycle per second) the slope of the power curve could be used for small angular oscillations, which in general produced lower values. For higher frequency oscillation, currents were induced in the field damper windings, which tend to stiffen the spring action and produced values under load higher than the conventional method.

C. Concordia developed results of calculations for damping and synchronizing torque coefficients of synchronous machines over a wide range of parameters [25]. The torques were calculated by the formulas given by R. H. Park [22]. Concordia, from the continued interest in damping and synchronizing torques, demonstrated that the equations given by Park are practically as simple and convenient to use as any of the numerous approximations that had been proposed from time to time.

It is common practice in stability studies to provide a means of adding $D$ damping that is proportional to speed or slip. $D$ is is the per unit damping coefficient used to compute a 
damping torque $T_{d}[5]$ defined as

$$
T_{d}=D \omega_{\Delta u} \quad(\text { per unit })
$$

The value used $D$ depends greatly on on the kind of generator model used and particularly on the modeling of the amortisseur windings. The value of $D$ depends on the units. When torque is calculated $M W$ then the slip $\omega_{\Delta}$ in $p u$ becomes equation 2.139.

$$
T_{d}=\left(S_{B} D\right) \omega_{\Delta u} \quad(M W)
$$

It is also common to see the slip calculated in Hertz $f_{\Delta} H z$, as written in equation 2.140.

$$
T_{d}=\left(S_{B} \frac{D}{f_{r}}\right) f_{\Delta}=D^{\prime} f_{\Delta} \quad(M W)
$$

Where $S_{B}$ is three-phase MVA base, $f_{r}$ is the base frequency in $H z$, an $f_{\Delta}$ is the slip in $H z$. A value sometimes used is $D^{\prime}$ in equation 2.140 is

$$
D^{\prime}=\frac{P_{G}}{f_{r}} \quad\left(\frac{M W}{H z}\right)
$$

Where $P_{G}$ is the scheduled power generated in $M W$. This corresponds to the damping Coefficient $D$ seen in equation 2.142 .

$$
D=\frac{P_{G}}{S_{B}} \quad(\text { per unit })
$$

In the early days when prime movers consisted mostly of reciprocating machines the pulsating torque made parallel operation difficult. This was successfully solved by damper windings in that the damper winding absorbed the energy of oscillation between machines and prevented the oscillations from becoming cumulative. 
During a transient, other extraneous torques are developed in a synchronous machine. The most important component is associated with the damper windings. While these torques are usually small in magnitude, their effect on stability is not negligible [5]. The most important effects are the following.

- Positive-sequence damping results from the interaction between the positive-sequence air gap flux and the rotor windings, particularly the damper windings. This effect is beneficial since it tends to reduce the magnitude of the machine oscillations, especially after the first swing. It is usually assumed to be proportional to the slip frequency.

- Negative-sequence braking results from interaction between negative-sequence air gap flux during asymmetrical faults and the damper windings. Negative-sequence torque is always retarding the rotor. Its magnitude is significant only when the rotor damper winding resistance is high.

- The dc braking is produced by the $d c$ component of the armature current during faults, which induces currents in the rotor winding of fundamental frequency. their interaction produces a torque that is always retarding the rotor.

Westinghouse engineers in 1944 [11] provided and excellent general discussion of units and dimensional analysis of how inertia constants and acceleration were determined in the past.

The inertia of a synchronous machine is a significant factor in stability since it affects the natural period of oscillation, or time required to reach a point beyond which recovery is 
possible. Knowledge of the inertia constant $H$ is a required for determination of acceleration and deceleration of the rotor. It represents the stored energy per kva and can be computed from the moment of inertia and speed by the following expression:

$$
H=\frac{0.231\left(W R^{2}\right)(r p m)^{2} 10^{-6}}{k v a} \quad \text { cycles per minute }
$$

where

- $W R^{2}$ is the moment of inertia in pounds-feet squared

- rpm is the speed in revolutions per minute

The range of inertia constants for various types of synchronous machines is illustrated in Figure 2.13.

The accelerating power depends on the initial operating conditions and upon the difference of input and output, including the effects of losses. Thus, for a synchronous generator the accelerating power, which is variable $\Delta P$ is written in equation 2.144 .

$$
\Delta P=P_{i}-\left(P_{0}+L\right)
$$

Where $P_{i}$ is the mechanical input power, $P_{0}$ is the electrical output, and $L$ is the total losses.

The natural frequency of undamped electro-mechanical oscillation for a synchronous machine connected to an infinite bus and shaft connected to reciprocating machinery is written in equation 2.145 .

$$
f_{n}=\frac{35200}{n} \sqrt{\frac{P_{r} f}{W R^{2}}}
$$




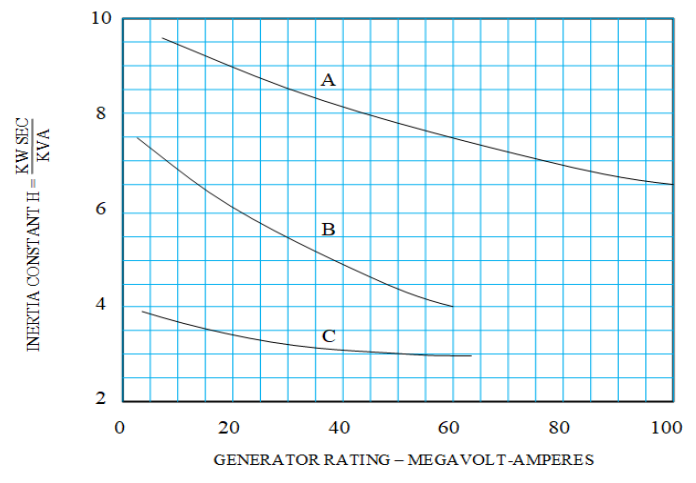

In er tia con stants of large turbogen erators, turbine included

A - 1,800 rpm condensing

$\mathrm{B}-3,600 \mathrm{rpm}$ condensing

$\mathrm{C}-3,600 \mathrm{rpm}$ noncondensing

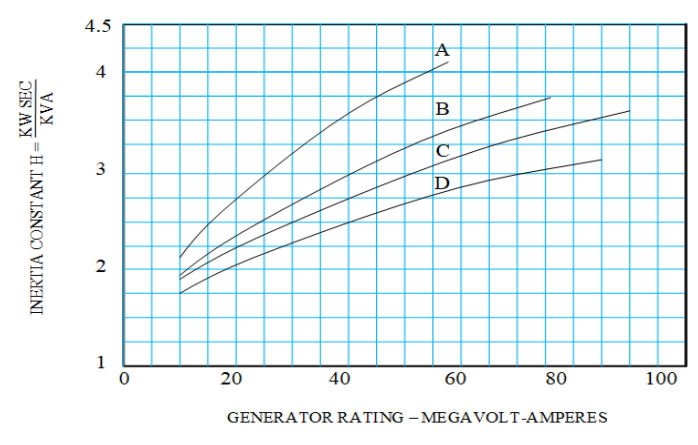

Inertia con stants of large ver tical-type water-wh eel generation, including allowance of 15 per cent for waterwheels
A $-450-514 \mathrm{rpm}$
B $-200-400 \mathrm{rpm}$
C - 138-180 rpm
B - 80-120 rpm

Figure 2.13: Inertia Constants from 1937 [9]

where

- $f_{n}=$ Natural frequency in cycle per minute

- $n$ = speed of machine in revolutions per minute

- $P_{r}=$ Synchronizing power

- $f=$ Frequency of circuit in cycles per second 
- $W R^{2}=$ Moment of inertia of a synchronous machine and shaft connected prime mover or load, in pounds foot squared

The synchronizing power is the power at synchronous speed corresponding to the torque developed at the air gap between the armature and the field. The synchronizing coefficient $P_{r}$ is determined by dividing the shaft power in $k W$ by the corresponding angular displacement of the rotor in electrical radians.

The displacement angle of the rotor for a given current power is written in equation 2.146. where

$$
\delta=\tan ^{-1} \frac{\bar{I} x_{q} \cos \theta}{E_{t}+\bar{I} \sin \theta}
$$

- $\delta=$ Rotor displacement angle in electrical radians

- $\bar{I}=$ Per-unit armature current

- $E_{t}=$ Per-unit armature terminal voltage

- $\theta=$ Power-factor angle

- $x_{q}=$ Per-unit quadrature-axis synchronous reactance

With the inertia constant $H$, and the accelerating power or decelerating power $\Delta P$ it is possible to calculate the acceleration as written in equation 2.147 .

$$
\alpha=\frac{(180)(f)(\Delta P)}{(H)(k v a)}
$$


where $\alpha$ is the acceleration or deceleration in electrical degrees per second per second, $f$ is the system frequency in cycles per second, $H$ is the inertia constant in kilowatt-seconds per $k v a$ as calculated by equation 2.143 .

- $\mathrm{f}=$ system frequency in cycles per second

- $\Delta P$ is the kilowatts available for acceleration (or deceleration)

- $\mathrm{H}=$ inertia constant in kilowatts-seconds per $k v a$ as calculated by equation 2.143.

Angle-time or swing curves could then be calculated by means the step-by-step procedure.

A typical form used historically was created by Westinghouse engineers, and is illustrated in Table 2.4. Among the series of calculation required to present in tabular form, references were often made to figures and models like one illustrated in Figure 2.11. This would be used for details of method approximating acceleration,, velocity, and angle changes. Below is the Step-by-Step Form as seen in 1944.

Solution

Machine Nos.

Total Kva

Machine, $W R^{2}=$

$\mathrm{H}=\ldots$ stored energy, kilowatt-seconds per kva

Acceleration, $\alpha=\frac{180(f)(\Delta P)}{(H)(k v a)}=k \Delta P$

$$
k=\frac{180 f}{(H)(k v a)}=
$$




$$
P_{\text {Mech. }}=\ldots \text { kilowatts, mechanical input }
$$

\begin{tabular}{|c|c|c|c|c|c|c|c|c|c|c|c|}
\hline \multicolumn{12}{|c|}{ Step-by-Step Calculations written in tabular form } \\
\hline$(1)$ & $(2)$ & (3) & $(4)$ & $(5)$ & (6) & (7) & $(8)$ & $(9)$ & (10) & (11) & (12) \\
\hline $\begin{array}{c}\text { Time } \\
\mathrm{sec}\end{array}$ & $\begin{array}{l}\text { angle } \theta \\
\text { Degrees }\end{array}$ & $\begin{array}{l}\text { Elec. } \\
\text { Output } \\
\text { plus } \\
\text { losses* } \\
\text { kw }\end{array}$ & $\begin{array}{c}\Delta P, \mathrm{kw} \\
\text { Mechanical } \\
\text { Input } \\
\text { minus } \\
\text { (Electrical } \\
\text { Output plus } \\
\text { losses) } \\
\end{array}$ & $\begin{array}{l}\text { Accle., } \alpha \\
\text { Degrees per } \\
\text { Sec per } \\
\text { sec }\end{array}$ & $\begin{array}{c}\text { Accel. } \\
\text { Time } \\
\text { Increment }\end{array}$ & $\begin{array}{c}\text { Vel. change } \\
\Delta \omega, \\
\text { Degrees } \\
\text { per sec }\end{array}$ & $\begin{array}{l}\text { Velocity } \\
\omega, \\
\text { Degrees } \\
\text { per sec }\end{array}$ & $\begin{array}{c}\text { Angle } \\
\text { Time } \\
\text { Increment }\end{array}$ & $\begin{array}{l}\text { Angular } \\
\text { Change for } \\
\text { Machine } \\
\text { Under Con- } \\
\text { sideration } \\
\Delta \theta \\
\text { degrees } \\
\end{array}$ & $\begin{array}{c}\text { Angular } \\
\text { Change for } \\
\text { for } \\
\text { other } \\
\text { Machine } \\
\Delta \theta^{\prime}, \\
\text { Degrees }\end{array}$ & $\begin{array}{c}\text { Final } \\
\text { Angle } \\
\text { Degrees }\end{array}$ \\
\hline- & - & - & $P_{\text {Mech }}-(3)$ & $(4) x k$ & - & (5) $x(6)$ & $(7)+(8)_{n-1}$ & - & (8) $x(9)$ & $* *$ & $(2)+(10)+(11)$ \\
\hline $\begin{array}{l}0.0 \\
0.1 \\
0.2 \\
0.3-\end{array}$ & $\begin{array}{l}\theta_{0} \\
\theta_{1} \\
\theta_{2} \\
\theta_{3} \\
\end{array}$ & $\begin{array}{c}P_{0} \\
P_{1} \\
P_{2} \\
P_{3-} \\
\end{array}$ & $\begin{array}{l}P_{\text {Mech }}-P_{0} \\
P_{\text {Mech }}-P_{1} \\
P_{\text {Mech }}-P_{2} \\
P_{\text {Mech }}-P_{3-} \\
\end{array}$ & $\begin{array}{c}k \Delta P_{0}=\alpha_{0+} \\
k \Delta P_{0}=\alpha_{1} \\
k \Delta P_{0}=\alpha_{2} \\
k \Delta P_{0}=\alpha_{3-}\end{array}$ & $\begin{array}{c}\Delta / 2 \\
\Delta t \\
\Delta t \\
\Delta t / 2\end{array}$ & $\begin{array}{c}\Delta \omega_{1} \\
\Delta \omega_{2} \\
\Delta \omega_{3} \\
\mathbf{V}_{e} \\
\end{array}$ & $\begin{array}{c}\omega_{0}+\Delta \omega_{1}=\omega_{1} \\
\omega_{1}+\Delta \omega_{1}=\omega_{2} \\
\omega_{2}+\Delta \omega_{1}=\omega_{3} \\
\omega_{3}+V_{e}\end{array}$ & $\begin{array}{l}\Delta t \\
\Delta t \\
\Delta t \\
-\end{array}$ & $\begin{array}{l}\omega_{1} \Delta t=\Delta \theta_{1} \\
\omega_{2} \Delta t=\Delta \theta_{2} \\
\omega_{3} \Delta t=\Delta \theta_{3}\end{array}$ & $\begin{array}{l}\Delta \theta_{1}^{\prime} \\
\Delta \theta_{2}^{\prime} \\
\Delta \theta_{3}^{\prime}\end{array}$ & $\begin{array}{l}\theta_{0}+\Delta \theta_{1}+\Delta \theta_{1}^{\prime}=\theta_{1} \\
\theta_{1}+\Delta \theta_{2}+\Delta \theta_{2}^{\prime}=\theta_{2} \\
\theta_{2}+\Delta \theta_{3}+\Delta \theta_{3}^{\prime}=\theta_{3}\end{array}$ \\
\hline $\begin{array}{c}0.3+ \\
0.4 \\
0.5 \\
\end{array}$ & $\begin{array}{l}\theta_{3} \\
\theta_{4} \\
\theta_{5} \\
\end{array}$ & $\begin{array}{c}P_{3+} \\
P_{4}\end{array}$ & $\begin{array}{c}P_{\text {Mech }}-P_{3+} \\
P_{M e c h}-P_{4}\end{array}$ & $\begin{array}{c}k \Delta P_{0}=\alpha_{3+} \\
k \Delta P_{0}=\alpha_{4}\end{array}$ & $\begin{array}{c}\Delta t / 2 \\
\Delta t\end{array}$ & $\begin{array}{l}\Delta \omega_{4} \\
\Delta \omega_{5}\end{array}$ & $\begin{array}{c}\omega_{3}+\left(V_{e}+\Delta \omega_{4}\right)=\omega_{4} \\
\omega_{4}+\Delta \omega_{5}=\omega_{5}\end{array}$ & $\begin{array}{l}\Delta t \\
\Delta t\end{array}$ & $\begin{array}{l}\omega_{4} \Delta t=\Delta \theta_{4} \\
\omega_{5} \Delta t=\Delta \theta_{5}\end{array}$ & $\begin{array}{l}\Delta \theta_{4}^{\prime} \\
\Delta \theta_{5}^{\prime}\end{array}$ & $\begin{array}{l}\theta_{3}+\Delta \theta_{4}+\Delta \theta_{4}^{\prime}=\theta_{4} \\
\theta_{4}+\Delta \theta_{5}+\Delta \theta_{5}^{\prime}=\theta_{5}\end{array}$ \\
\hline
\end{tabular}

Table 2.1: Suggested Form for Step-by-Step Angle-Time Calculations [11].

\subsection{PMU background}

Historically, C.P. Steinmetz developed the phasor vector notation, where the sine-function of time is represented by a constant numerical quantity, instead of an independent variable "time". Thus, a complex number representing a sinusoidal function whose amplitude $A$, angular frequency $\omega$, initial phase $\theta$ are time-variant. Making use of the polar coordinate system on a complex plane, where the vector is formed by its imaginary and real parts rotating around the origin. This was presented in the article, "Complex Quantities and Their Uses in Electrical Engineering," [26], and presented at the Proceedings of the International Electrical Congress held in the Chicago, August of 1893.

Time synchronized phasors, or synchrophasors, are calculated from data samples using a common time signal, as referenced in IEEE C37.118.1 [27] for synchrophasors. The origins of the synchrophasor evolved from computer systems dedicated to substation protection and control data logging functionality. New applications were developed that could utilize 
the microprocessor power, specifically a microcomputer based Symmetrical Component Distance Relay (SCDR) [28]. Synchrophasors are measured using a Phasor Measurement Unit (PMU. These devices can be stand-alone or enabled from a protective device. This device was first developed by Phadke \& Thorp at Virginia Tech [29] in Blacksburg, VA in the 1988 .

PMUs require a common and accurate correct timing reference. A convenient timing reference, described in [27], establishes the relationship between the Coordinated Universal Time (UTC) time scale and the phase of the reference cosine wave. The Global Positioning System (GPS) is the source of time synchronization for synchrophasors. GPS receivers transmit UTC as an offset, which allows local time to be utilized with a satellite clock by using a local signal type such as Inter-range Instrumentation Group Time Code Format B (IRIG-B). The IRIG-B modulates the required signal at the accuracy for the PMU.

Using the received IRIG-B signal to time-stamp the acquired voltage and current values, PMUs estimate phasors that are synchronized with UTC [30], as illustrated in Figure 2.14.

We represent the sinusoidal voltage signal as shown in equation 2.148.

$$
V_{x}(t)=V_{x} \cos \left(2 \pi f t+\delta_{x}\right)=V_{x} \cos \left(\omega t+\delta_{x}\right)
$$

- $V_{x}=$ Signal amplitude in volts

- $\delta_{x}=$ Angle in radians

- $f=$ Frequency in Hertz 


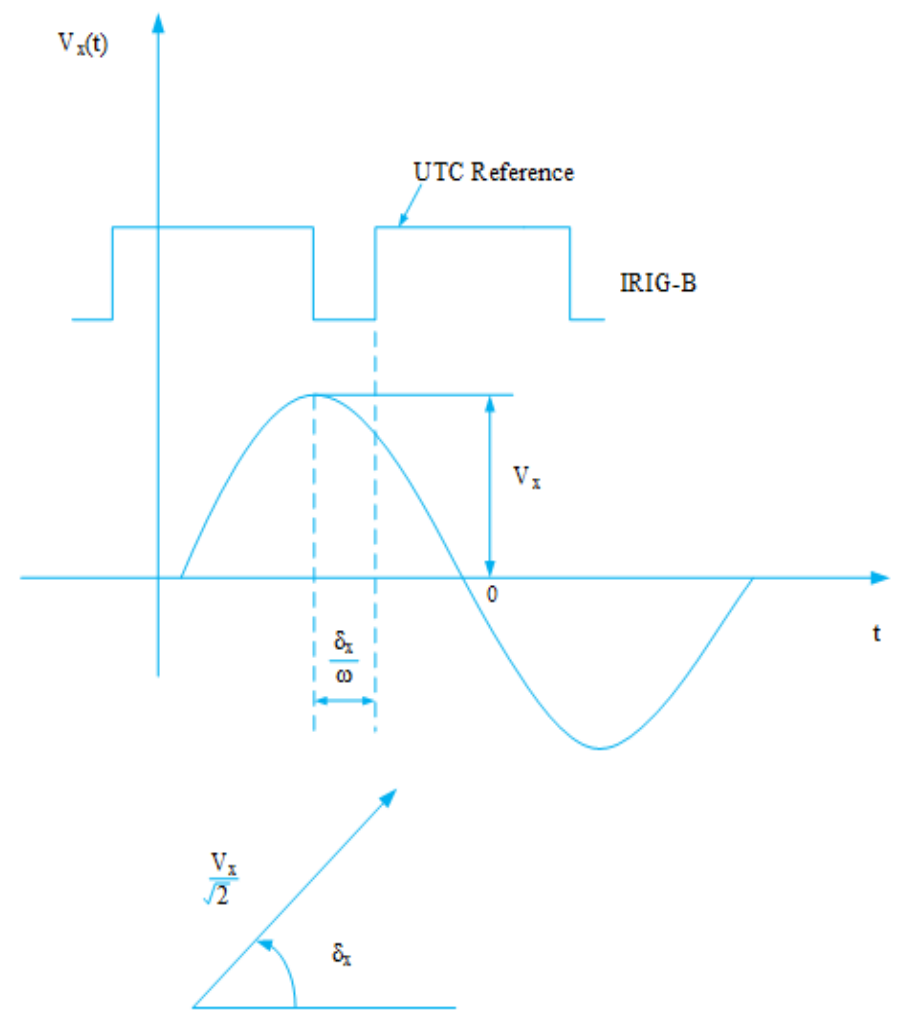

Figure 2.14: Synchrophasor illustration using the UTC reference [30]

- $\omega=$ Angular frequency in radians per second

- $t=$ Time in seconds

PMUs provide time-synchronized information on phasor angles at different locations within a power system, including real-time frequency and rate of change of frequency, as illustrated in Figure 2.15.

The functionality of a PMU enables it to measure positive-sequence data. Positive sequence voltage phasor information is a parameter of vital significance, whereby the collection of all positive sequence voltage phasors constitutes the state-vector of a power system [28]. Figure 2.16, illustrates two sinusoidal signals, which represent voltages at 


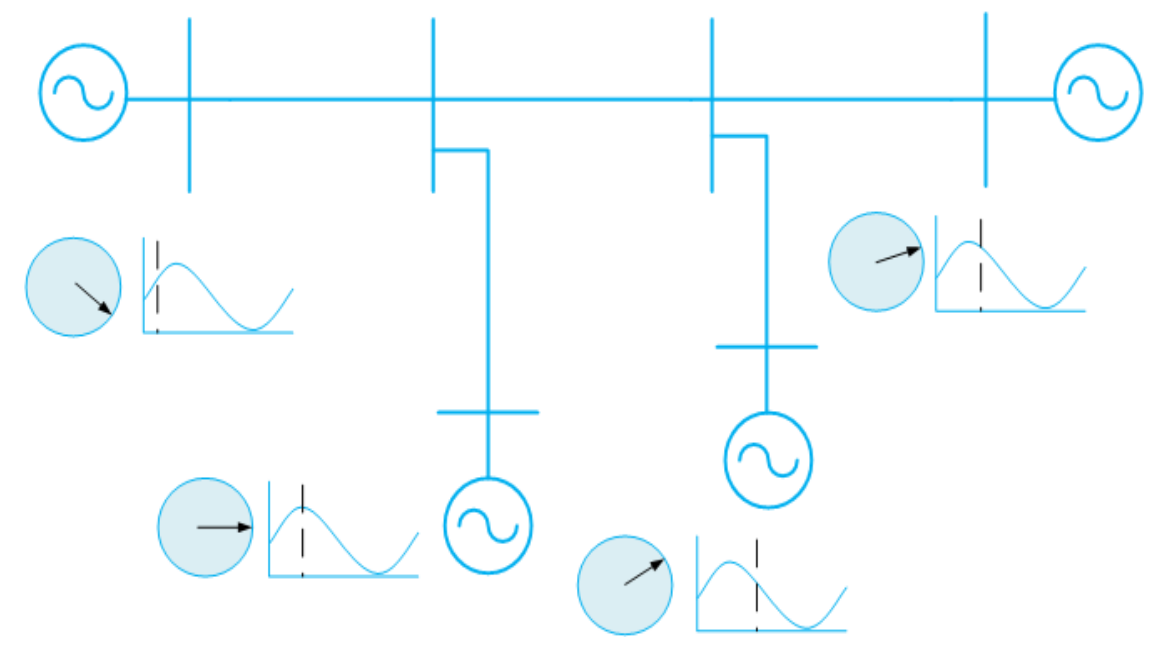

Figure 2.15: Synchrophasors associate phasor measurements to an absolute time reference across the power system [30]

different power system locations. Signal angles can be measured with respect to a timesynchronized common reference, where the angle difference $\Delta \delta$ can be calculated.

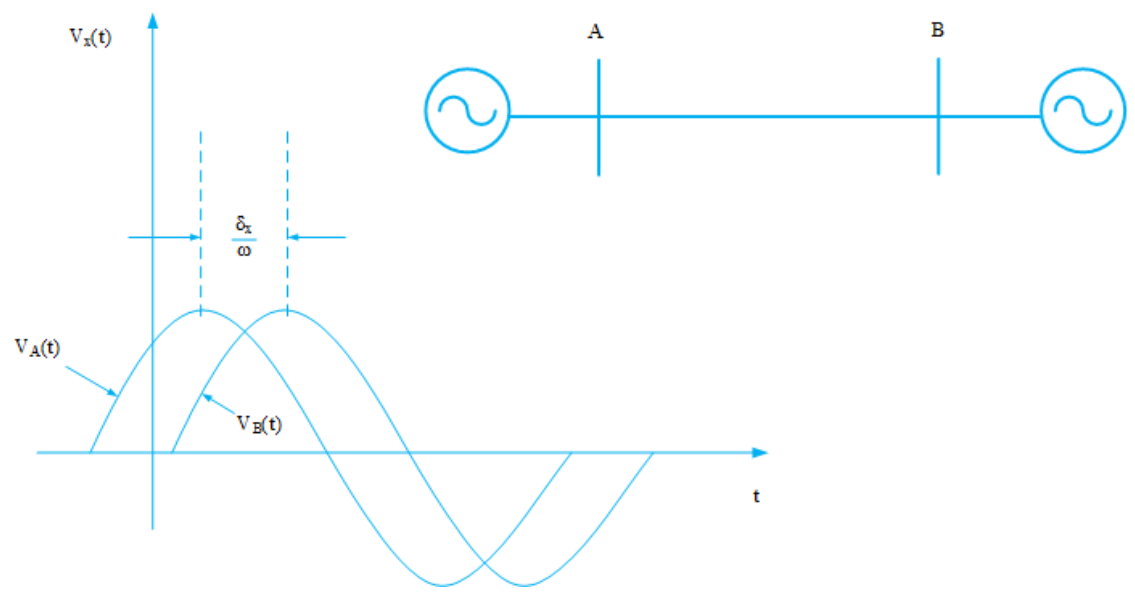

Figure 2.16: Voltage signal angles at different locations [30]

Frequency is significant in real-time phasor measurements. Phasors are a steady-state concept applied at a single frequency. In an actual network, system frequency changes constantly, based on system dynamics. 
The swing equation determines generator speed, which in turn determines the frequency at the generator output. When each generator swing equation reaches equilibrium, the operating frequency is the same on all points of the network.

During a power system disturbance, the phasor angle and frequency undergo local changes. Equation 2.149 expresses frequency $f$ as a function of nominal frequency $f_{0}$ and the angle $\delta$.

$$
f=f_{0}+\frac{1}{2 \pi} \frac{d \delta}{d t}
$$

When measuring frequency, synchrophasors are always computed in relation to the system nominal frequency $\left(f_{0}\right)$ [27]. The rate of change of frequency (ROCOF) is shown in equation 2.150 .

$$
\operatorname{ROCOF}(t)=\frac{d f(t)}{d t}
$$

The rate at which grid frequency changes is directly measured at different locations were PMUs are deployed. The data obtained are significant, and useful in determining the coefficients of the swing equation, which directly characterize the power system. 


\section{Results \& Analysis}

\subsection{How to derive $D \&$ H from PMU data}

Damping $D$ and inertia $H$ are calculated using the rate of change of frequency ROCOF PMU data. The data analyzed in this report represent thirteen separate transmission-level events, which include both $230 \mathrm{kV}$ and $500 \mathrm{kV}$ systems. Data from each event were measured by three separate PMUs deployed at different locations. Time-stamped, positive-sequence voltage and frequency data were measured at a rate 60 samples per second. The total data for each event captured 10,800 time-stamped measurements. These data included data prior to, during, and after the event signal where a second-order system equation method could be used to analyze the oscillations of a disturbance.

Referencing the solution of the swing equation in Section 2.1.9 characteristic equations, the linearized swing equations, 2.107 and 2.108, are rewritten below as equations 3.1 and 3.2. Angular momentum is written in terms of the inertia constant $H$ and damping $D$ to create the standard expression shown in 3.1. The standard form of the second-order equation differential equation for analyzing the dynamics of a linear system is shown in equation 3.2.

$$
\begin{aligned}
& \frac{H}{\pi f_{0}} \frac{d^{2} \delta}{d t^{2}}+D \frac{d \delta}{d t}+P_{s}=0 \\
& \frac{d^{2} \delta}{d t^{2}}+2 \zeta \omega_{n} \frac{d \delta}{d t}+\omega_{n}^{2}=0
\end{aligned}
$$


Equation 3.1 is divided by the synchronizing power coefficient $\left(P_{s}\right)$ and equation 3.2 is multiplied by $\left(1 / \omega_{n}^{2}\right)$, further reduced and rewritten as equations 3.3 and 3.4 , where both the inertia constant and damping coefficient are in terms of $P_{s}$.

$$
\begin{array}{cc}
\frac{D}{P_{s}}=\frac{2 \zeta}{\omega_{n}} & \left(\frac{1}{\frac{r a d}{s}}\right) \\
\frac{H}{P_{s}}=\frac{\pi f_{0}}{\omega_{n}^{2}} & \left(\frac{1}{\left(\frac{r a d}{s}\right)^{2}}\right)
\end{array}
$$

As previously mentioned, frequency is significant in time-synchronized phasor measurements for characterizing a power system. From the second-order equations, both natural frequency $\omega_{n}$ in equation 3.5 , and the damping ratio $\zeta$ in equation 3.6 can be determined by the rate of change of frequency. These coefficients are used to calculate $D$ and $H$ in the fit filter program.

$$
\begin{gathered}
\omega_{n}=\sqrt{\frac{\pi f_{s}}{H} P_{s}} \quad\left(\frac{\text { rad }}{s}\right) \\
\zeta=\frac{D}{2} \sqrt{\frac{\pi f_{s}}{H P_{s}}} \quad(\text { unit }- \text { less })
\end{gathered}
$$

\subsection{Fit Filter Program}

The fit filter program analyzes a system disturbances, i.e. events, and reads the rate of change frequency data through an undamped second-order system equation to determine the coefficients of the swing equation. Source code for the fit program is persistently archived within the PSU Library's PDXScholar website [31]. 
In the first function of the program, the data file for each event is categorized in a datastore file for handling tall arrays. This is configured to read csv files from three PMUs per each event that will be run in fit column function.

The fit column function finds the best fitting second-order exponential function for the data sets. The data are time-stamped from the PMUs so the first column in each csv file coincides with each consecutive rate of change of frequency. This filters the decay fit data, which will be run through a separate function that determines the event period.

Peak amplitude is then determined, where it compares the data set maximum values and inverse maximum values. The function then returns the highest values and indexes it to be used in another function that finds the event period.

All the data in an event time period are parameterized by a moving window that is established from the maximum amplitude. A window size of 50 samples is correlated with a moving average index of 0.002 in length to establish the event period. The convergence of the moving mean average was determined to be better for these data than using five $\tau$ due to the length of the data. Typical event periods are small, ranging from a few cycles to a couple seconds. The event files used for this analysis were on average 10,800 measurements in length at 60 samples per second, requiring the program to run through 180 seconds of data.

After the event period is established, the filter data function defines the numerator and denominator of a rational transfer function. Visual inspection of initial data sets found a window size of three gave a reasonable fit. Where the window size is the numerator 
$\left(\frac{1 / \text { windowsize }}{1}\right)$ in the rational transfer function.

The exponential coefficients and functions are parameterized into decay fit filtered data. This function correlates time, peak amplitude, and Goodness of Fit (GOF) for a second order system for damping ratios between zero to one to calculate zeta and the natural frequency. The damping ratio is indexed between 0.001 and 1 with a step size of 0.001 and $\omega_{n}$, which is related to tau $\tau$ and is calculated by $\frac{4}{\zeta \tau}$. The data are then run through the second differential equation, shown in equation 3.7 .

$$
\text { Decay }=A e^{\left(-\zeta \omega_{n} t\right)} \cdot \cos \left(\omega_{n} \sqrt{1-\zeta^{2}} \cdot t\right)
$$

The coefficients that are calculated from this program include $\zeta, \omega_{n}$, Amplitude and GOF.

\subsection{PMU Test Data}

The PMU data used for this analysis were provided by BPA, Portland, OR. These data are persistently archived within the PSU Library's PDXScholar website [31].

The event files are transmission-level events. However, the PMU locations were not identified. There are thirteen separate events. As previously mentioned, the events were measured by three PMUs at a rate of sixty samples per second, acquiring positive-sequence voltage and frequency data. There are a total of thirty-nine event signals used for this analysis. The event data are stored using the csv file format. The following list are the events recorded: 


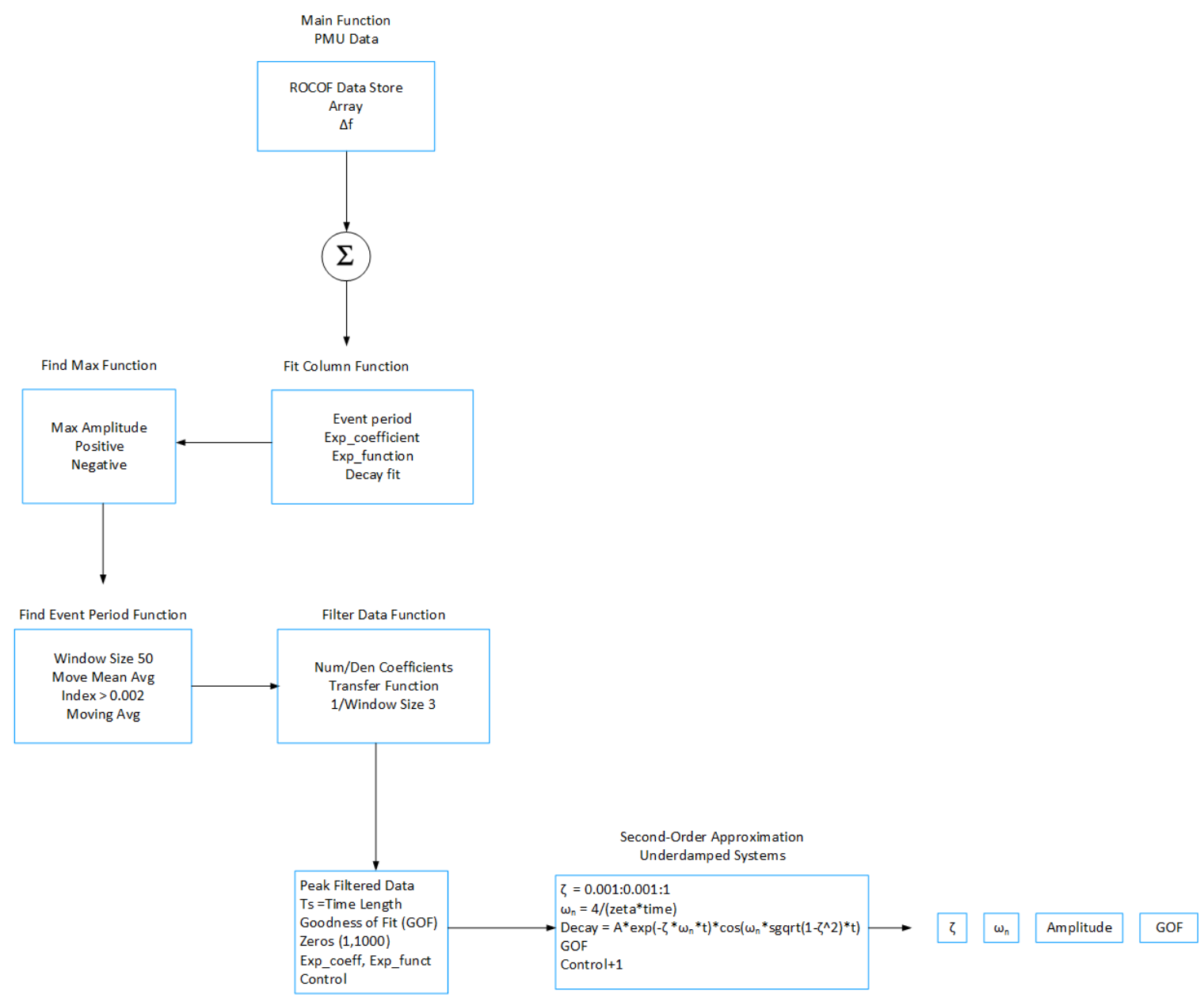

Figure 3.1: Flow diagram for the fit filter program that analyzes ROCOF PMU data to determine the coefficients of a second-order undamped system.

- Event 1: Outage on BPA Direct Current (DC) line going from the Dalles, OR area down to California

- Event 2: Line trip that led to a 2600 MW gen drop

- Event 3: Insertion of the $1400 \mathrm{MW}$ brake resistor at Chief Joseph

- Event 4: Generator outage in Montana

- Event 5: Generator outage in the Lower Columbia region 
- Event 6: Fault on a couple of lines at one station, causing a loss of over $2200 \mathrm{MW}$

- Event 7: Line trip leading to a $1400 \mathrm{MW}$ generation drop

- Event 8: Line trip on a major line bringing generation from Montana

- Event 9: Outage on BPA DC line (similar to event 1 from the previous set)

- Event 10: Trip/reclose/trip on a line in the lower Columbia region

- Event 11: Insertion of the 1400 MW brake resistor at Chief Joseph

- Event 12: Insertion of the $1400 \mathrm{MW}$ brake resistor at Chief Joseph

- Event 13: Insertion of the 1400 MW brake resistor at Chief Joseph

\subsection{Sample preparation prior to using fit filter program}

The PMU data in each of the event files contains measurements prior, during, and after the event. Non event data includes frequencies and noise that limit the effectiveness of the fit filter program. The data sets are 10,800 measurements in length, compared to the small signal of the actual event, which are 600-800 measurements long. Sample preparation requires truncating the data from 10,800 down to the event lengths at the defined period.

In Figure 3.2(C), the complete signal of Event 3-PMU 1 is illustrated, which is a 10,800 measurement data set. When all 10,800 measurements are run through the fit program the event signal registered approximately 780 measurements in length, as shown in Figure 3.2(B). Evaluating Figure 3.2(B) further, the signal illustrates that the red line of the fit 
program does not follow the PMU data, shown in blue, close enough to provide useful approximations of the system coefficients.

With a visual inspection of the small signal length, the next step is to zoom into the signal where the peak amplitude and signal decay occurs, as illustrated in Figure 3.2(D). The fit program determines positive and negative peak amplitudes as the starting point of calculations for the second-order undamped system. To provide a consistent methodology, the event is measured just prior to the peak amplitude to capture the signal at the start of decay, and at the end, when the event signal is no longer decaying. If additional peaks occur after the initial peak, the start of the event signal can be adjusted to provide a more accurate PMU data set. In this example, the event is determined to be from 3861 to 4398 measurements, or 767 samples in length.

Consistent evaluation of event sample lengths is important. As illustrated in Figure 3.2(C), where the correct initial start of the signal was used, but also included the signal length out to 1400 measurements, which consisted of excessive noise at the end of the signal decay, and thus, reducing the goodness of fit. Conversely, if the start of the sample length is measured past the peak amplitude, or past the final peak amplitude if there are multiple peaks, then the fit will be greatly reduced. The sinusoidal fit will distort and take on a parabolic shape.

The final step is to use the truncated signal. In this example the PMU data set is truncated to approximately 800 measurements, and the results are illustrated in Figure 3.2(E), for 
the event signal, and figure 3.2(F), matching the complete signal length evaluated. The fit program calculated useful results that correlated time, peak amplitude, and goodness of fit for a second-order undamped system, whereby the coefficients of the swing equation could be determined by time-synchronized measurements. The results for Event 3-PMU 1, are listed in Table 3.1.

\begin{tabular}{|c|c|c|c|c|}
\hline \multicolumn{5}{|c|}{ Event 3: All Data vs. Truncated PMU Data } \\
\hline & $\begin{array}{c}\text { All } \\
\text { Standard } \\
\text { Deviation }\end{array}$ & Average & $\begin{array}{c}\text { Truncated } \\
\text { Standard } \\
\text { Deviation }\end{array}$ & Average \\
\hline$\zeta$ & 0.0153 & 0.133 & 0.00125 & 0.146 \\
\hline$\omega_{n}$ & 0.000414 & 0.0471 & 0.000115 & 0.0466 \\
\hline$G O F$ & 0.675 & -0.326 & 0.117 & 0.334 \\
\hline$D / P_{s}$ & 0.604 & 5.662 & 0.0723 & 7.198 \\
\hline$H / P_{s}$ & 1490 & 85128 & 643 & 112025 \\
\hline Tau & 18.42 & 161.50 & 1.3 & 168 \\
\hline
\end{tabular}

Table 3.1: Statistical Comparison Event 3: All PMU Data vs. Truncated PMU Data

\subsection{Signal Outliers}

The method developed in this paper to determine the coefficients of a second-order undamped system does not work for all PMU event signals. Each of the thirteen events were measured by three PMUs at separate locations, for a total of thirty-nine event data sets. Of those thirty-nine events, the fit program method produced good results from twenty-seven. Twelve PMU data sets produced less than ideal results, which are considered outliers.

Events that include multiple high-frequency transients and other oscillatory signals are difficult to adequately calculate signal decay in a fit program. Multiple spikes in amplitude can throw off the start of an event period. Additionally, multiple steps in the sinusoidal signal within the signal decay can distort the moving window, and indexing of fit step 

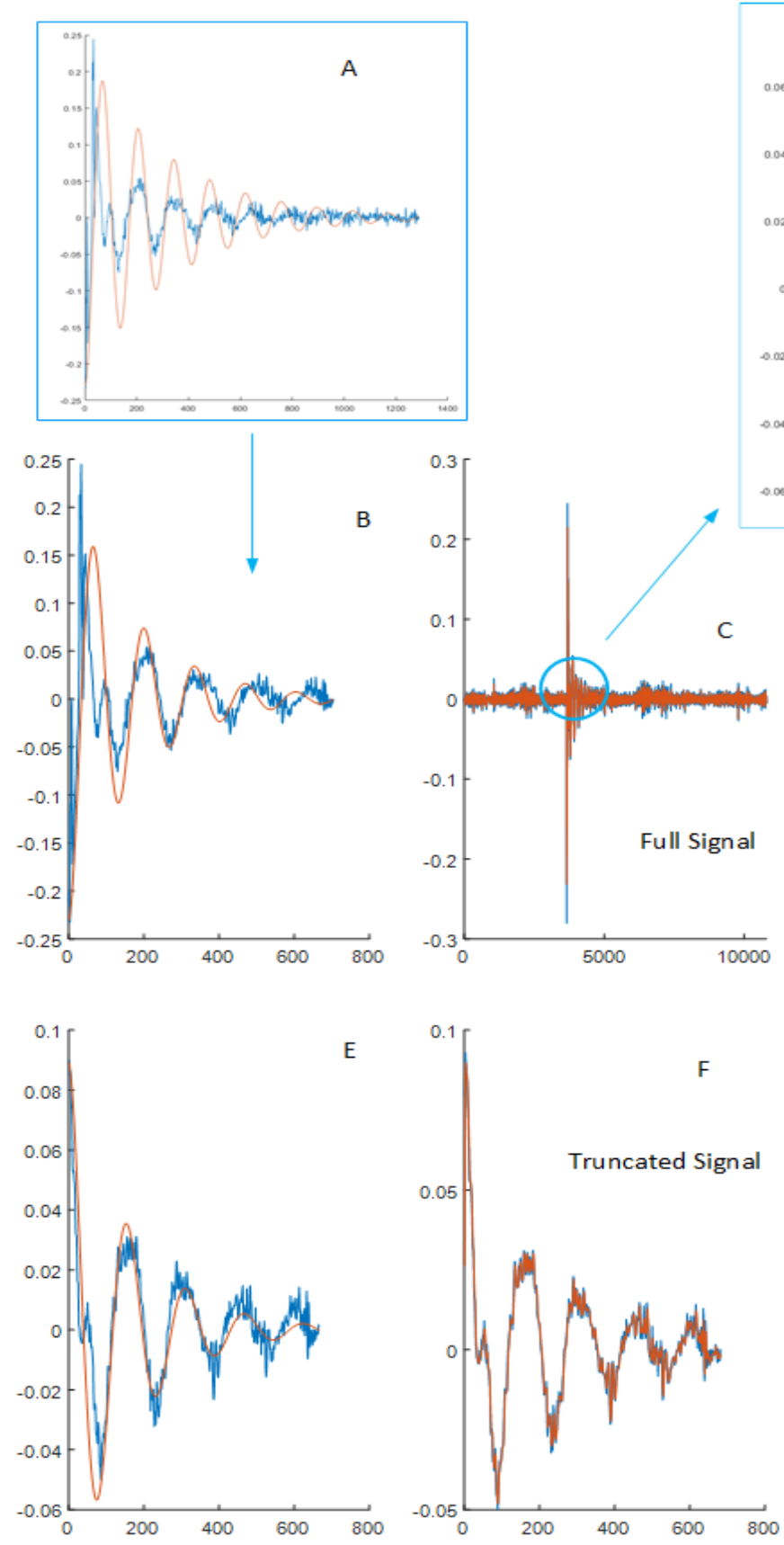

Figure 3.2: PMU data sample preparation for fit filter program. Truncate 10,800 PMU measurements to determine event period, from initial start through end of decay.

increments. These effects, coupled with excessive oscillatory signal response can distort the final measurement of signal decay. 
Figure 3.3, evaluating the signal from Event 1-PMU 1, illustrates how the fit program could not adequately fit the signal decay. The full data set is approximately 7500 measurements in length, where the actual event period length was visually approximated to be 850 measurements. The event signal was then evaluated between 2800 and 3450 measurements. The next step was to truncate the front-half of the signal down to a 4500 data set length. From inspection, the event signal was present in wave form, but distorted. When the signal was further truncated past the approximate end of decay, using a 800 data set length, there was little to no improvement in the goodness of fit.

\subsection{Plots}

The analysis of the data includes the system coefficients, goodness of fit, and the calculated results for $D, H$ and $\tau$. The event signals were plotted in the fit filter program, and then data sets for each event were plotted in Excel X-Y plots. An excel block-and-whisker plot was created to illustrate the statistical comparison between all PMU data to truncated PMU data.

In Figure 3.4, $\zeta, \omega_{n}, G O F, D / P_{s}, H / P_{s}$ and $\tau$ are plots of all PMU data (in blue) for thirteen events compared to all truncated data (in red). From initial observation, the plots illustrate that truncated data provides a tighter grouping of results, which produces more refined values.

Figure 3.4(A), illustrates the damping ratio $\zeta$, which is referenced by equation 3.6. The coefficient $\zeta$ has a standard deviation of 0.191 for all data compared to 0.140 for truncated data. A consistent value for $\zeta$ is 0.146 , where 0.161 is the average. This value determines 

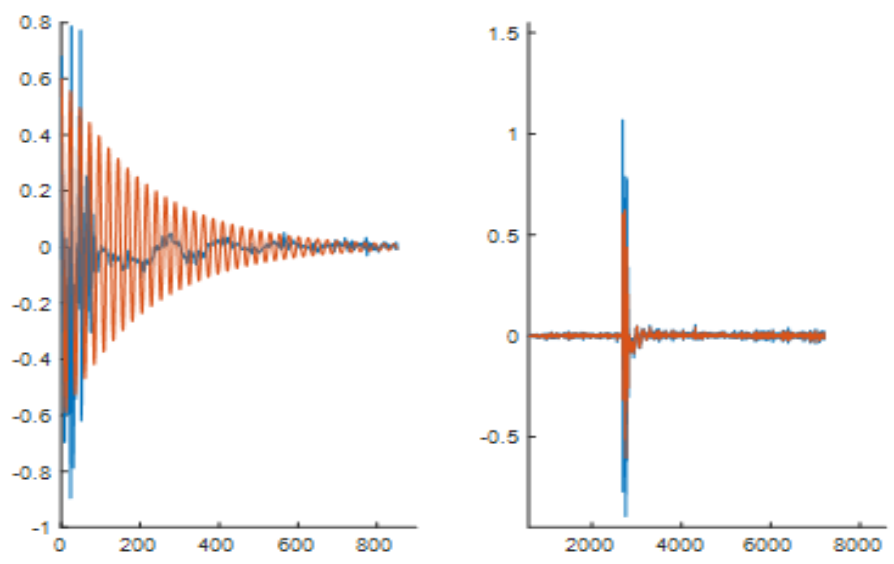

7500 Data Set
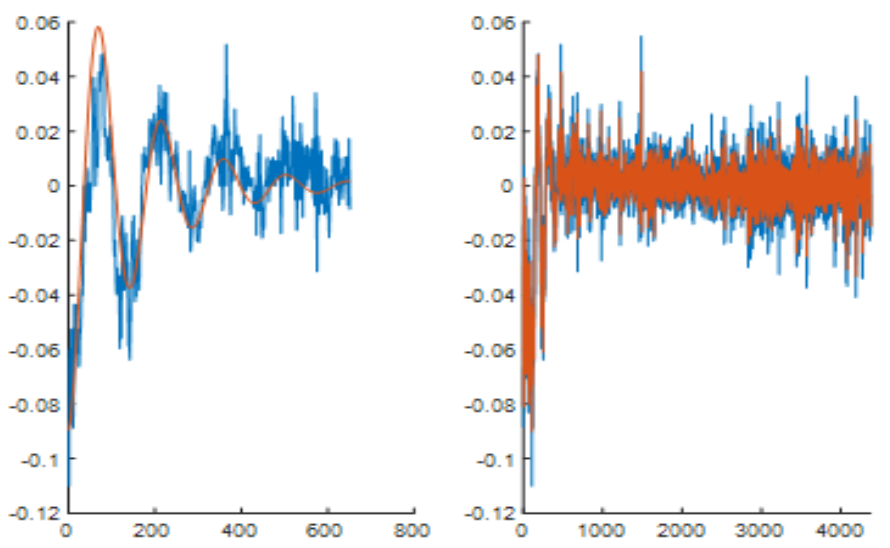

4500 Data Set
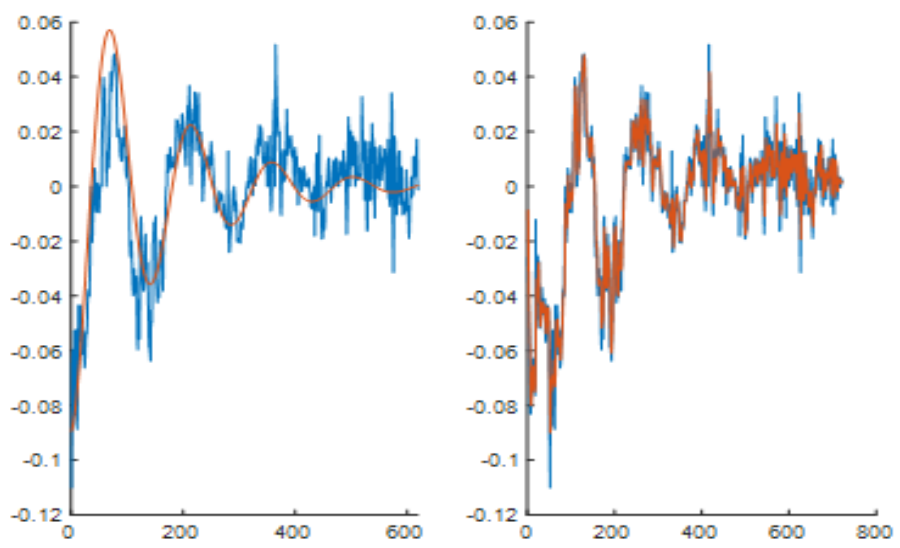

800 Data Set

Figure 3.3: Example of a PMU data outlier. Data set identified and truncated to initial start of event. No improvement made in $G O F$ results when truncating from 4500 to 800 data set period. 
the rate of decay of the amplitude of oscillation. Typical values $\zeta$ range from 0.137 to 0.150. Figure 3.4(B) illustrates the undamped natural frequency $\omega_{n}$ of oscillation, which is referenced by equation 3.5 . The coefficient $\omega_{n}$ has a standard deviation of 0.110 compared to 0.075 for truncated. A consistent value for $\omega_{n}$ is 0.0405 , where 0.0697 is the average. Typical values for $\omega_{n}$ range from 0.0309 to 0.048 . As the synchronizing power coefficient $P_{s}$ increases, the natural frequency increases, and the damping ratio decreases. An increase in the damping coefficient $D$ increase the damping ratio, whereas and increase in the inertia constant decreases both $\omega_{n}$ and $\zeta$.

Figure 3.4(c) illustrates the goodness of fit $(G O F)$. The goodness of fit has a standard deviation of 0.904 compared to 0.750 for truncated. A consistent value for $G O F$ is 0.170 where -0.236 is the average. Goodness of fit correlates the decay fit filtered data with time and peak amplitude. The closer to zero, the better the fit with the oscillation of decay. Typical values range from 0.001 to 0.400 . Figure 3.4(D) illustrates the damping coefficient $D / P_{s}$. The damping coefficient has a standard deviation of 25.410 compared to 9.657 for truncated. A consistent value for $D / P_{s}$ is 7.20 where 7.34 is the average. Typical values range from 5.00 to 8.00. Figure 3.4(E) illustrates the inertia constant $H / P_{s}$. The inertia constant has a standard deviation of 192952 compared to 40278 for truncated. A consistent value for $H / P_{s}$ is 114761 where 83522 is the average. Typical values range from 72000 to 124000 . Figure 3.4(F) illustrates the time constant $\tau$. The time constant has a standard deviation of 47.805 compared to 37.935 for truncated. A consistent value for $\tau$ is 169 where 136.71 is the average. The time constant relates $\zeta$ and $\omega_{n}$ to the settling time of exponential 
decay. Typical values for $\tau$ range from 140 to 180. Data for statistical comparisons are listed in Table 3.2.
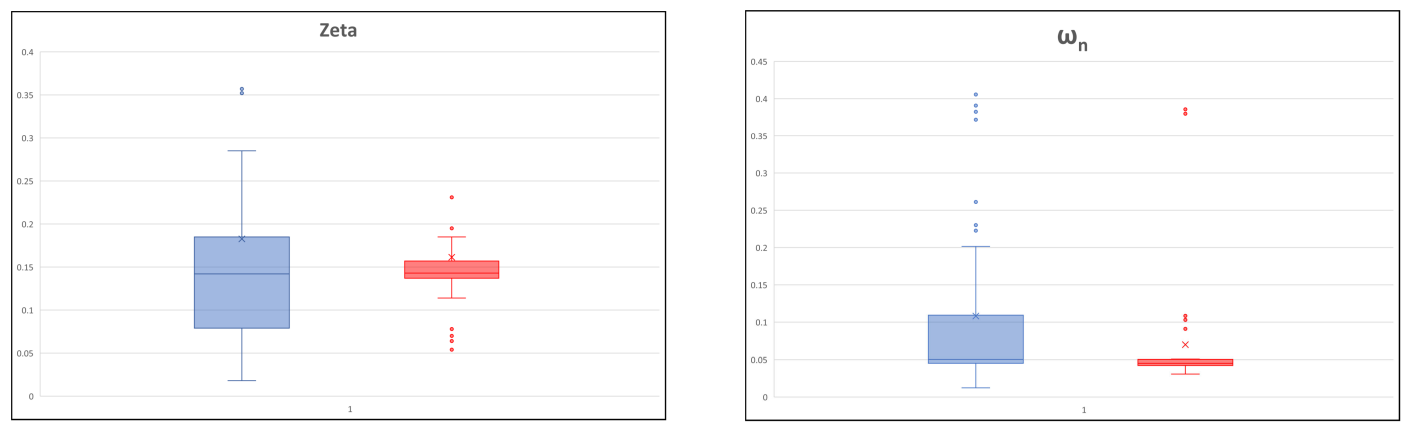

[A]

$[\mathrm{B}]$
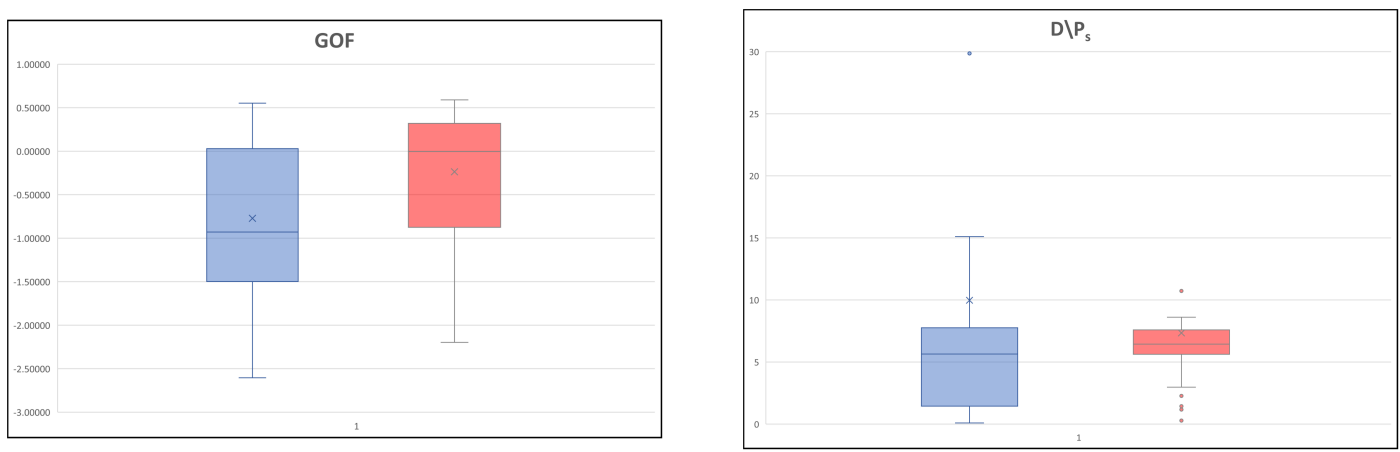

$[\mathrm{C}]$

[D]
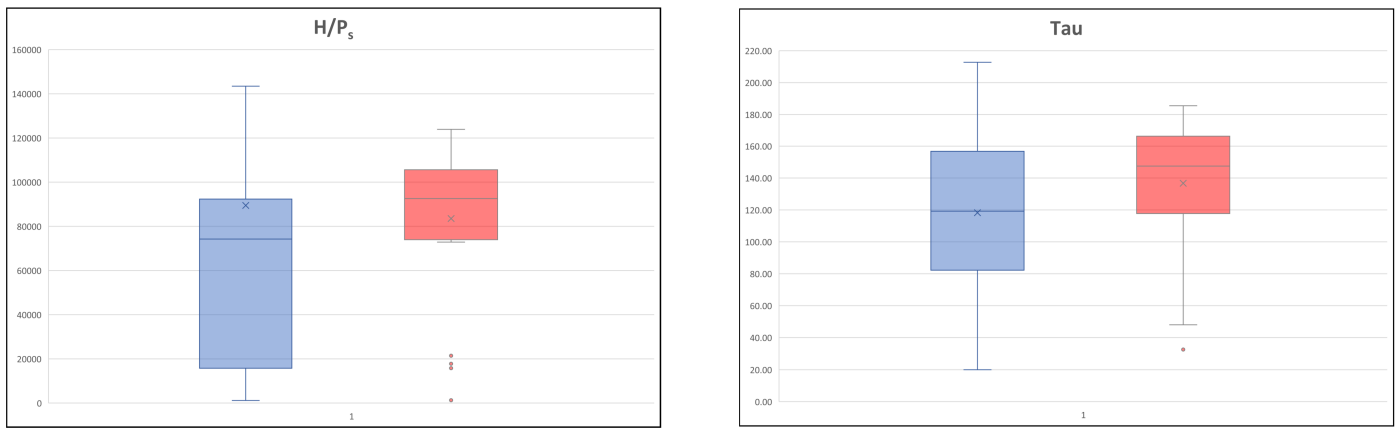

[E]

$[\mathrm{F}]$

Figure 3.4: Statistical box-and-whisker plots of all PMU data. Untruncated data (blue) compared to truncated (red) data. BOX encompasses exclusive median. ' $\mathrm{X}$ ' marks the average. Dots show the Outliers. Line is the Mean. Whiskers are the upper and lower quartiles. 


\begin{tabular}{ccccc}
\hline \multicolumn{3}{c}{ All PMU Data vs. ALL Truncated PMU Data } \\
\hline \multicolumn{4}{c}{ All } & \multicolumn{3}{c}{$\begin{array}{c}\text { Truncated } \\
\text { Standard } \\
\text { Deviation }\end{array}$} & Average & $\begin{array}{c}\text { Standard } \\
\text { Deviation }\end{array}$ & Average \\
\hline$\zeta$ & 0.191 & 0.182 & 0.140 & 0.161 \\
$\omega_{n}$ & 0.110 & 0.108 & 0.075 & 0.069 \\
$G O F$ & 0.904 & -0.771 & 0.750 & -0.236 \\
$D / P_{s}$ & 25.410 & 9.976 & 9.657 & 7.342 \\
$H / P_{s}$ & 192,952 & 89,471 & 40,278 & 83,522 \\
Tau & 47.805 & 118.28 & 37.935 & 136.71 \\
\hline
\end{tabular}

Table 3.2: Statistical Comparison of All PMU Data vs. Truncated PMU Data 


\section{Discussion}

\subsection{Potential Sources of Error}

As previously mentioned, the peak amplitude, which indicates the start of the event, can throw off the fit program if there are secondary spikes in amplitude. Adjustments in signal start time, as well as the end of signal decay need to be made. This may take several attempts to provide a reasonable fit.

High frequency oscillations can reduce the effectiveness of the fit program. Some signals can not be evaluated with this fit program method.

Depending on the PMU measurements of a specific event, the indexing steps and moving mean average may need to be adjusted.

Depending on number of data sets measured, i.e., voltages and frequency, the PMU data being evaluated must be set up to correlate with the exact number of columns to read of rate of change of frequency. Additionally, the time header needs to be included. 


\subsection{Improvements}

The sample preparation requires visual inspection and iterative steps to determine an event signal length. A function could be developed in the fit filter program that would automate the process to determine the event period length, if the event period length is not predetermined in the PMU data set.

Additional functionality could be realized if an additional function could be inserted that would automatically read the number of PMUs in the data set and determine columns of rate of change of frequency correlated to time. The current fit program has a function that has to be manually directed to those columns of data.

\subsection{BPA Chief Jo Example}

In 1973, Bonneville Power Administration (BPA) constructed a 1400 MW dynamic braking resistor at Chief Joseph substation in north central Washington. The braking resistor was designed to enhance stability in the Pacific Northwest, and increase capacity of the Pacific Northwest-Southwest inter-tie.

Faults near the large concentrations of BPA generation tend to accelerate the entire Pacific Northwest generating area. This produces angular swings between the Pacific Northwest and the interconnected systems, and can cause separation of the interconnection points although each area remains intact. When the Pacific Northwest experiences a severe fault, a post-fault speed deviation between that area and its neighbors will result, which is 
proportional to the product of the fault accelerating power and the fault duration. If this speed deviation is great enough, the tie-line synchronizing power may be insufficient to maintain transient stability [32].

The dynamic brake resistor is designed to dissipate $1400 \mathrm{MW}$ of power when energized to $240 \mathrm{kV}$, and can withstand a three second run time. Appropriate application of the resistor brake at Chief Joseph immediately after a fault helps retard the Pacific Northwest generating area sufficiently to prevent tripping of the Pacific Northwest-Southwest inter-tie [32].

The primary purpose of the Chief Joseph braking resistor is to restrain the generating area when accelerated by a severe fault to prevent loss of synchronism. In addition to the benefits of improving stability in the operating area for first and second-swing fault applications, the application of determining the system signal response can be performed by temporarily inserting the dynamic brake, and measuring the signal response, essentially pinging the system with a delta load function. Earlier methods of using Chief Joseph dynamic brake to measure signal response included inserting and removing the brake after several cycles. The velocity is measured from the accelerating power, steady-sate frequency and $H$. The relative velocity of a generator directly relates to the stored energy in the inertia of the generator. Damping can then be measured from the brake output power response decay from its initial power level, and associated time constants to evaluate the sinusoids with the swing equation. Current methods include probing signal injection after Chief Joseph brake insertion by measuring frequency sine wave deviations and ambient noise ring-downs with PMU data [33]. 


\subsection{Discussion of Results}

Measuring the signal response by dynamic brake insertion has a cost associated with its use, from power requirements to engineering analysis. Test equipment that directly measures synchronous generator output, and transient stability software for modeling analysis are expensive. The benefit is real-time analysis, but at a cost.

Originally the stability of power systems was calculated principally in terms of maximum power, which could be be carried under steady-load conditions. However, a much more direct and accurate method of approach to the problem is to calculate the effect of disturbances directly, that is, to calculate stability under transient conditions [34].

This thesis presents a unique method of modeling the swing equation using timesynchronized phasor measurements from electrical transients, which produces useful coefficients of the swing equation at minimal cost. Our challenge is that we have to wait for an event to occur to obtain PMU measurements. With the event of a large disturbance that excites system dynamics, valuable data are obtained from PMUs with useful coefficients, which are available around the power system.

As shown in Table 4.1, twenty-seven separate PMU data sets recorded measurements that provided consistent damping coefficients $\frac{D}{P_{s}}$ and inertia constants $\frac{H}{P_{s}}$ after the measurements were truncated, which measure between 5 to 8 for $D$, and between 72000 to 123000 for $H$.

In addition to the $\frac{D}{P_{s}}$ and $\frac{H}{P_{s}}$ values meeting established parameters, the values illustrated 
in Table 4.1 provide a unique and useful comparison of the relationship between $\frac{D}{P_{s}}$ and $\frac{H}{P_{s}}$. When comparing the truncated values for $\frac{D}{P_{s}}$ and $\frac{H}{P_{s}}$, the values are noticeably close for each event, as compared to $\frac{D}{P_{s}}$ and $\frac{H}{P_{s}}$ values between their respective events. This comparison shows that $\frac{D}{P_{s}}$ and $\frac{H}{P_{s}}$ differ through time, depending on the configuration of the power system.

Further illustrating the effectiveness of the method used in this work to prepare data sets for evaluation, ideally, working with data sets that contain just the event measurements would remove the truncating step to determine the event period. However, regardless of the length of PMU measurements, a data set can be prepared for evaluation with this method.

From the thirty-nine PMU data sets evaluated, twelve PMU data sets did not provide data that yielded adequate results. This includes event No.4 and event No.8, where both were line trips from a generator outage in Montana. This effectively excluded six PMU data sets. The remaining six PMUs did not produce adequate results, because of PMU observability, in that they were not optimally placed to capture good measurements for those events. These twelve outlier PMU data sets are illustrated in Table 4.2. However, the method proved to yield consistent results for the other PMUs in those same events. Table 4.3 provides an overview of the results, which illustrates the twenty-seven PMU data sets that yielded consistent results for determining the coefficients of a second-order undamped system.

Furthermore, this works demonstrates that readily available PMU data from an event 


\begin{tabular}{lcccc}
\hline \multicolumn{1}{c}{ Damping Coefficient $\frac{D}{P_{s}}$ and Inertia Constant $\frac{H}{P_{s}}$} & for 27 PMUs \\
\hline & & $\frac{D}{P_{S}}$ & & $\frac{H}{P_{S}}$ \\
Event-PMU & All Data & Truncated & All Data & Truncated \\
\hline E1-PMU1 & 0.137 & 6.68 & 2764 & 97547 \\
E1-PMU2 & 7.094 & 6.09 & 126381 & 95811 \\
E1-PMU3 & 0.356 & 6.87 & 3560 & 100219 \\
\hline E2-PMU1 & 5.718 & 6.07 & 98637 & 92565 \\
E2-PMU2 & 5.643 & 5.97 & 97566 & 90655 \\
E2-PMU3 & 7.758 & 7.44 & 106757 & 120856 \\
\hline E3-PMU1 & 5.154 & 7.20 & 85489 & 114761 \\
E3-PMU2 & 5.320 & 7.10 & 86746 & 113195 \\
E3-PMU3 & 6.510 & 7.28 & 83149 & 114120 \\
\hline E5-PMU1 & 0.496 & 7.90 & 4642 & 105632 \\
E5-PMU2 & 29.85 & 7.90 & 56286 & 108272 \\
\hline E6-PMU1 & 0.233 & 7.97 & 3803 & 88486 \\
E6-PMU2 & 4.736 & 7.77 & 45756 & 83110 \\
E6-PMU3 & 0.093 & 6.66 & 1146 & 91617 \\
\hline E7-PMU1 & 15.10 & 6.44 & 84339 & 95620 \\
E7-PMU2 & 9.606 & 7.07 & 92350 & 95680 \\
\hline E9-PMU1 & 7.564 & 6.33 & 97849 & 100750 \\
E9-PMU2 & 6.899 & 6.50 & 102424 & 103099 \\
E9-PMU3 & 8.385 & 6.33 & 96813 & 100750 \\
\hline E10-PMU1 & 5.551 & 8.04 & 84634 & 116184 \\
E10-PMU2 & 9.710 & 7.59 & 143468 & 123933 \\
E10-PMU3 & 0.118 & 8.03 & 1364 & 111652 \\
\hline E11-PMU1 & 5.635 & 5.62 & 74233 & 72877 \\
E11-PMU3 & 10.39 & 5.66 & 62714 & 73941 \\
\hline E12-PMU3 & 7.273 & 5.81 & 72831 & 78817 \\
\hline E13-PMU1 & 6,903 & 5.84 & 86246 & 85550 \\
E13-PMU3 & 7.388 & 5.92 & 83034 & 86083 \\
\hline
\end{tabular}

Table 4.1: Statistical Comparison of 27 PMUs that yielded consistent results, illustrating useful truncated values between 5 to 8 for $\frac{D}{P_{s}}$, and 72000 to 123000 for $\frac{H}{P_{s}}$. 


\begin{tabular}{ccccc}
\hline \multicolumn{5}{c}{ Damping Coefficient $\frac{D}{P_{s}}$ and Inertia Constant $\frac{H}{P_{s}}$ for 12 PMUs } \\
\hline Event-PMU & All Data & $\begin{array}{c}\frac{D}{P_{S}} \\
\text { Truncated }\end{array}$ & All Data & $\begin{array}{c}\frac{H}{P_{S}} \\
\text { Truncated }\end{array}$ \\
\hline E4-PMU1 & 2.780 & 3.11 & 21551 & 21413 \\
E4-PMU2 & 4.011 & 2.97 & 21457 & 21594 \\
E4-PMU3 & 163.00 & 65.00 & 1252077 & 199105 \\
\hline E5-PMU3 & 14.18 & 10.72 & 76551 & 101595 \\
\hline E7-PMU3 & 10.615 & 8.61 & 87046 & 91931 \\
\hline E8-PMU1 & 0.329 & 0.284 & 1289 & 1306 \\
E8-PMU2 & 3.872 & 2.272 & 16332 & 17776 \\
E8-PMU3 & 0.455 & 0.279 & 1234 & 1266 \\
\hline E11-PMU2 & 1.444 & 1.43 & 15762 & 16036 \\
\hline E12-PMU1 & 6.745 & 4.821 & 72474 & 84297 \\
E12-PMU2 & 1.435 & 1.171 & 15558 & 15789 \\
\hline E13-PMU2 & 1.572 & 1.53 & 23051 & 22767 \\
\hline
\end{tabular}

Table 4.2: 12 PMUs that did not yield adequate results, $\frac{D}{P_{s}}$ and $\frac{H}{P_{s}}$.

can provide useful data to determine the coefficient of the swing equation, thus, providing a low-cost, simple, and reproducible method to evaluate the characteristics of a power system during transient conditions. 


\begin{tabular}{c|cccc|cc}
\hline \multicolumn{7}{c}{ Coefficients and Constants for 27 PMUs } \\
\hline Event & \multicolumn{1}{c}{$\omega_{n}$} \\
PMU & $\zeta$ & $\omega_{n}$ & $G O F$ & $\tau$ & $\frac{D}{P_{s}}$ & $\frac{H}{P_{s}}$ \\
\hline E1-PMU1 & 0.147 & 0.0439 & 0.396 & 154 & 6.68 & 97547 \\
E1-PMU2 & 0.135 & 0.0443 & 0.550 & 167 & 6.08 & 95811 \\
E1-PMU3 & 0.149 & 0.0433 & 0.175 & 154 & 6.87 & 100219 \\
E2-PMU1 & 0.137 & 0.0451 & 0.525 & 161 & 6.07 & 92565 \\
E2-PMU2 & 0.136 & 0.0456 & 0.580 & 161 & 5.96 & 90655 \\
E2-PMU3 & 0.147 & 0.0394 & -0.0433 & 172 & 7.44 & 120856 \\
E3-PMU1 & 0.146 & 0.0405 & 0.169 & 169 & 7.20 & 114761 \\
E3-PMU2 & 0.145 & 0.0408 & 0.396 & 169 & 7.10 & 113195 \\
E3-PMU3 & 0.148 & 0.0406 & 0.434 & 169 & 7.28 & 114120 \\
E5-PMU1 & 0.167 & 0.0422 & -2.197 & 141 & 7.90 & 105632 \\
E5-PMU2 & 0.165 & 0.0417 & -0.399 & 145 & 7.90 & 108272 \\
E6-PMU1 & 0.184 & 0.0461 & -0.003 & 117 & 7.97 & 88486 \\
E6-PMU2 & 0.185 & 0.0476 & 0.452 & 113 & 7.76 & 83110 \\
E6-PMU3 & 0.151 & 0.0453 & -0.099 & 146 & 6.65 & 91617 \\
E7-PMU1 & 0.143 & 0.0444 & -0.875 & 157 & 6.44 & 95620 \\
E7-PMU2 & 0.157 & 0.0443 & -1.224 & 143 & 7.07 & 95680 \\
E9-PMU1 & 0.137 & 0.0432 & -2.152 & 168 & 6.33 & 100750 \\
E9-PMU2 & 0.139 & 0.0427 & 0.365 & 168 & 6.50 & 103099 \\
E9-PMU3 & 0.137 & 0.0432 & -1.327 & 168 & 6.33 & 100750 \\
E10-PMU1 & 0.162 & 0.0402 & 0.307 & 153 & 8.04 & 116184 \\
E10-PMU2 & 0.148 & 0.0390 & 0.590 & 173 & 7.58 & 123933 \\
E10-PMU3 & 0.165 & 0.0410 & 0.163 & 147 & 8.03 & 111652 \\
E11-PMU1 & 0.143 & 0.0508 & 0.013 & 137 & 5.62 & 72877 \\
E11-PMU3 & 0.143 & 0.0509 & 0.319 & 138 & 5.66 & 73941 \\
E12-PMU3 & 0.142 & 0.0489 & 0.300 & 144 & 5.80 & 78817 \\
E13-PMU1 & 0.137 & 0.0469 & 0.031 & 155 & 5.83 & 85550 \\
E13-PMU3 & 0.138 & 0.0466 & 0.260 & 155 & 5.92 & 86083 \\
\hline
\end{tabular}

Table 4.3: Results of 27 PMUs, which include determined coefficients natural frequency $\omega_{n}$, damping ratio $\zeta$, the goodness of $G O F$, compared with calculated damping coefficients $\frac{D}{P_{s}}$ and Inertia constants $\frac{H}{P_{s}}$. 


\section{Conclusion}

The equation governing the motion of the rotor of a synchronous machine is based on an elementary principle of dynamics, where accelerating torque is the product of the moment of inertia of the rotor times its angular acceleration. During a disturbance, how the rotor will accelerate or decelerate is described in relative motion by the swing equation.

During transient conditions when a disturbance excites system dynamics, valuable data are obtained from PMUs with useful coefficients that are available around the system. The coefficients of the swing equation can be obtained by evaluating event period data sets PMU rate of change of frequency measurements. These coefficients are then used to calculate the important damping coefficient $D$ and inertia constant $H$, which characterize the restoring ability of a power system after a disturbance.

PMU data are readily available. However, obtaining event data is achieved by either purposefully inserting a dynamic resistor brake and obtaining measurements, like the BPA's dynamic brake at Chief Joseph substation, or waiting until an event occurs and obtaining PMU measurements that are observable for that system.

PMU data sets for events can include just the event period, or any number of measurements, in addition to the event period that is being evaluated. This work demonstrates an 
economical and effective method for determining the event period start, truncating large PMU data sets to determine an event period through end of decay to reduce variability and improve goodness of fit, when evaluating event samples through a fit filter program. The fit filter program reads the rate of change frequency data through an undamped second-order system equation to determine the coefficients of the swing equation.

This research uses archived PMU data obtained from BPA in order to illustrate the viability of this method. The event files are transmission-level events. There are thirteen separate events, which were measured by three PMUs at a rate of sixty samples per second, acquiring positive-sequence voltage and frequency data. There are a total of thirty-nine event signals used for this analysis. The event data are stored using the csv file format.

The rate of change of frequency data are evaluated through the fit filter program, where the filtered data are calculated by the undamped second-order equation, which yields the coefficients for damping ratio $\zeta$, natural frequency $\omega_{n}$, and goodness of fit GOF. These coefficients are then used to calculate $\frac{D}{P_{s}}, \frac{H}{P_{s}}$, and the time-constant $\tau$.

From the thirty-nine PMUs, twenty-seven PMUs produced data sets that provided consistent results. The outleirs are reviewed in the Discussion of Results, section 4.4, which clarify that two events from six PMUs were not able to obtain adequate measurements for the same type of event. The other six PMUs that did not produce good results were in a location that did not provide the necessary observability, or included high-frequency oscillations. 
The results of the typical consistent values for twenty seven PMUs are illustrated in Table 5.1, which include the evaluated coefficients (natural damping ratio $\zeta$, frequency $\omega_{n}$, goodness of fit (GOF) with calculated damping coefficients $\frac{D}{P_{s}}$ and Inertia constants $\frac{H}{P_{s}}$.

\begin{tabular}{cccc|cc}
\hline \multicolumn{6}{c}{ Typical Good Values for Coefficients and Constants } \\
\hline$\zeta$ & $\omega_{n}$ & $G O F$ & $\tau$ & $\frac{D}{P_{s}}$ & $\frac{H}{P_{s}}$ \\
\hline $0.137-0.150$ & $0.031-0.048$ & $0.001-0.400$ & $140-180$ & $5.00-8.00$ & $72000-124000$ \\
\hline
\end{tabular}

Table 5.1: Results of 27 PMUs, which include the evaluated coefficients damping ratio $\zeta$, natural frequency $\omega_{n}$, the goodness of $G O F$, with calculated damping coefficients $\frac{D}{P_{s}}$ and Inertia constants $\frac{H}{P_{s}}$.

This work successfully demonstrates a unique method of modeling the swing equation using time-synchronized phasors by using ROCOF from PMU data and a MATLAB analysis fit program to determine the second-order coefficients. These coefficients are used to calculate the damping coefficient $\frac{D}{P_{s}}$, and inertia constant $\frac{H}{P_{s}}$. These coefficients are necessary to create a practical swing equation.

The value obtained from these results illustrates an economical method for transient system analysis using readily available and useful coefficients that are necessary to create a practical swing equation

Future work could lead to improvements in the program functionality. By developing an automated process of truncating long PMU data sets, event periods can then be automatically determined. During the truncating of an event data set, multiple peak amplitude spikes are evaluated to determine when the actual event period starts. A function could be created to optimize initial start times just prior to the final peak amplitude, right before the signal 
decays. Additional functions could be developed to automatically adjust window sizes of transfer functions, and tune parameters of indexing to improve the goodness of fit. 


\section{Bibliography}

[1] R. Podmore and A. Germond. Development of Dynamic Equivalents for Transient Stability Studies. Electrical Power Research Institute, 1977.

[2] E.W. Kimbark. Power System Stability, Elements of Stability Calculations, volume I. John Wiley \& Sons, New York, NY, 1948.

[3] Technical Committee Annual Reports, A Generation of the American Institute of Electrical Engineers...1884-1924. Transactions of the American Institute of Electrical Engineers, 43(1):1058-1117, 1924.

[4] D. W. Stevenson. Elements of Power System Analysis, volume IV. Mcgraw-Hill International Book Company, Singapore, 1982.

[5] P. M. Anderson and A. A. Fouad. Power System Control and Stability. Wiley, Piscataway, NJ, 2003.

[6] R. D. Wilkins and C. F. Wagner. Further Studies of Transmission System Stability. Journal of the American Institute of Electrical Engineers, 45(4):374-383, 1926.

[7] R. Wilkins. Practical aspects of system stability. Journal of the American Institute of Electrical Engineers, 45(2):142-151, 1926. 
[8] R. J. C. Wood, L. F. Hunt, and S. B. Griscom. Transients Due to Short Circuits A Study of Tests Made on the Southern California Edison 220-kV System. Transactions of the American Institute of Electrical Engineers, 47(1):68-86, 1928.

[9] AIEE Subcommittee. First report on power system stability. Transactions of the American Institute of Electrical Engineers, 56(2):261-282, 1937.

[10] G. J. Grainger and W. D. Stevenson. Power System Analysis, volume I. McGraw-Hill, Hightstown, NJ, 1994.

[11] Central Station Engineers of Westinghouse. Electrical Transmission and Distribution Reference Book, volume I. Westinghouse, East Pittsburgh, PA, 1944.

[12] P. Kundur. Power System Stability and Control, volume I. McGraw-Hill, Hightstown, NJ, 1994.

[13] C. Concordia, S. B. Crary, and E. E. Parker. Effect of Prime Mover Speed Governing Characteristics on Power System Tie-line Power Swings. Transactions of the American Institute of Electrical Engineers, 60(6):559-567, 1941.

[14] S. B. Crary. Power System Stability, volume I. Wiley, New York, NY, 1945.

[15] C. L. Fortescue. Transmission Stability Analytical Discussion of Some Factors into the Problem. Transactions of the American Institute of Electrical Engineers, 44(9):984$1003,1925$. 
[16] R. P. Feynman, R. B. Leighton, and M. Sands. The Feynman Lectures On Physics, volume 1. Basic Books, New York, NY, 1963.

[17] H. W. Dommel and N. Sato. Bonneville Power Administration fast transient stability solutions. IEEE Transactions on Power Apparatus and Systems, 91(4):1643-1650, 1972.

[18] P. Denholm, T. Mai, R. W. Kenyon, B. Kropski, and M. O’Malley. Inertia and the Power Grid: A Guide without the Spin. U.S. Department of Energy, National Renewable Energy laboratory, pages 4-18, 2020.

[19] M. M. Liwschitz. Positive and Negative Damping in Synchronous Machines. Transactions of the American Institute of Electrical Engineers, 60(5):210-213, 1941.

[20] L. A. Kilgore and E. C. Whitney. Spring and Damping Coefficients of Synchronous Machines and Their Application. Transactions of the American Institute of Electrical Engineers, 69(1), 1950.

[21] R. E. Doherty and C. A. Nickle. Synchronous Machines I-An Extension of Blondel's Two-Reaction Theory. Transactions of the American Institute of Electrical Engineers, 45(10):912-947, 1926.

[22] R. H. Park. Two-reaction Theory of Synchronous Machines-II. Transactions of the American Institute of Electrical Engineers, 52(2):352-354, 1933.

[23] F. R. Longley. The Calculation of Alternator Swing Curves The Step-by-Step Method. Transactions of the American Institute of Electrical Engineers, 49(3):1129-1150, 1930. 
[24] S. B. Crary and M. L. Waring. Torque-Angle Characteristics of Synchronous Machines following System Disturbances. Transactions of the American Institute of Electrical Engineers, 51(4):764-773, 1932.

[25] C. Concordia. Synchronous Machine Damping and Synchronizing Torques. Transactions of the American Institute of Electrical Engineers, 70(1):731-737, 1951.

[26] C. P. Steinmetz. Complex Quantities and Their Uses in Electrical Engineering. IEC, Proceedings of IEC Chicago, August 21-25:33-75, 1893.

[27] IEEE Power Energy Society. Ieee Standard for Synchrophasor Measurements for Power Systems. The Institute of Electrical Engineers, 2011.

[28] A. G Phadke, J. S. Thorp, and M. G. Adamiak. A New measurement Technique for Tracking Voltage Phasors, Local System frequency, and Rate of Change of Frequency. IEEE Power Engineering Review, 3(5):1025-1038, 1983.

[29] A. G. Phadke and J. S. Thorp. History and applications of phasor measurements. In 2006 IEEE PES Power Systems Conference and Exposition, pages 331-335, 2006.

[30] E. O. Schweitzer and H. J. Altuve-Ferrer. Modern Solutions for Protection, Control, and Monitoring Electric Power Systems. Pullman, WA, 2010.

[31] R. Ferraro. Data From: Method of Modeling the Swing Equation Using Time Synchronized Measurements. Electrical and Computer Engineering Datasets, 2021. 
[32] M. L. Shelton, W. A. Mittelsdat, P. F. Winkelman, and W. J. Bellerby. Bonneville Power Administration 1400- MW Braking Resister. pages 602-611, 1974.

[33] J. Fullum. Pacific Northwest National Laboratory, 2019 Brake Tests: Initial Analysis. Presentation, 2019.

[34] R. H. Park and F. H. Bancker. System Stability as a Design Problem. Transactions of the American Institute of Electrical Engineers, 48(1):170-193, 1928. 
Appendix A: X-Y Plots

\section{A.1 X-Y Plots}



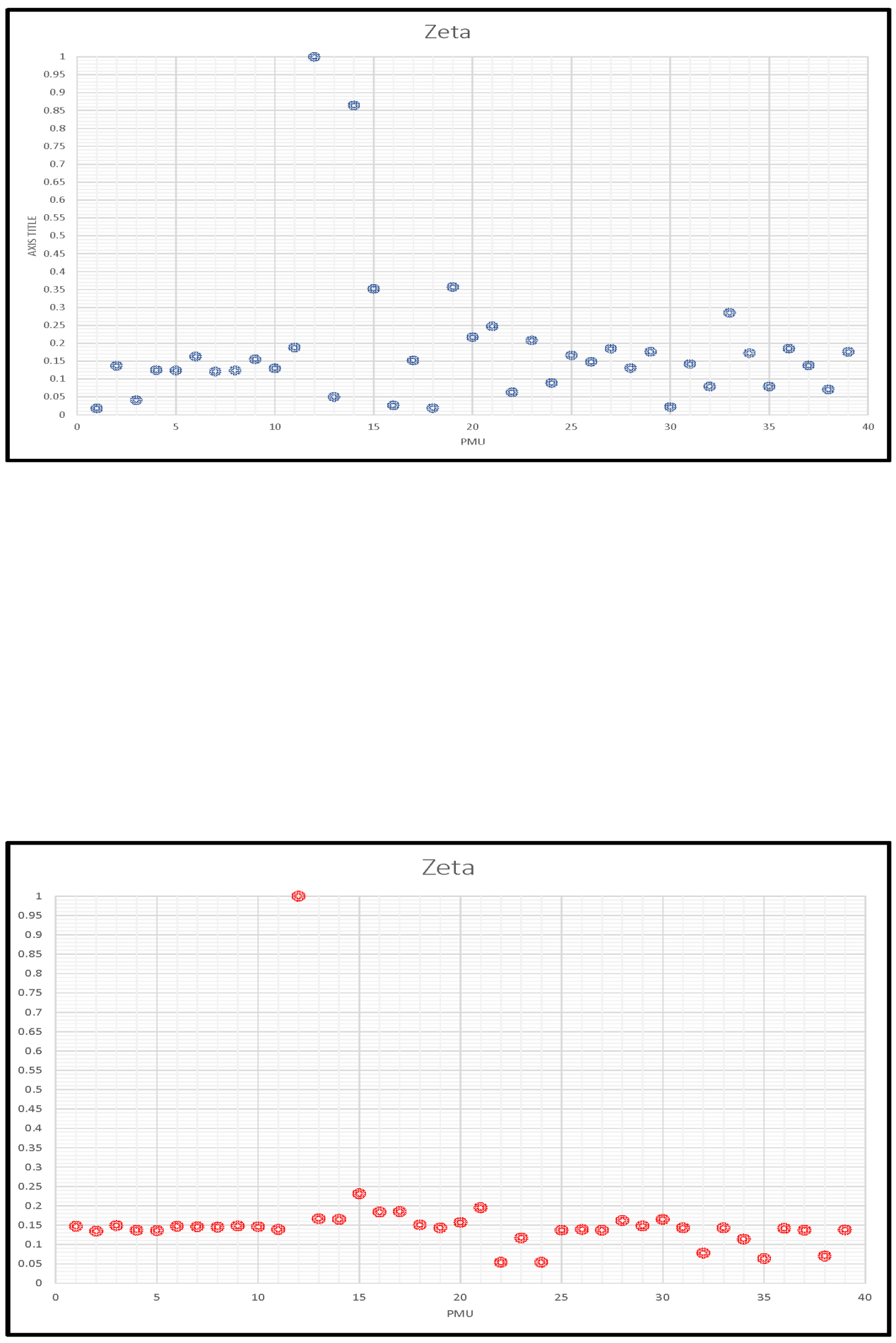

Figure A.1: All (blue) vs. Truncated (red) PMU Data-Zeta $(\zeta)$ 

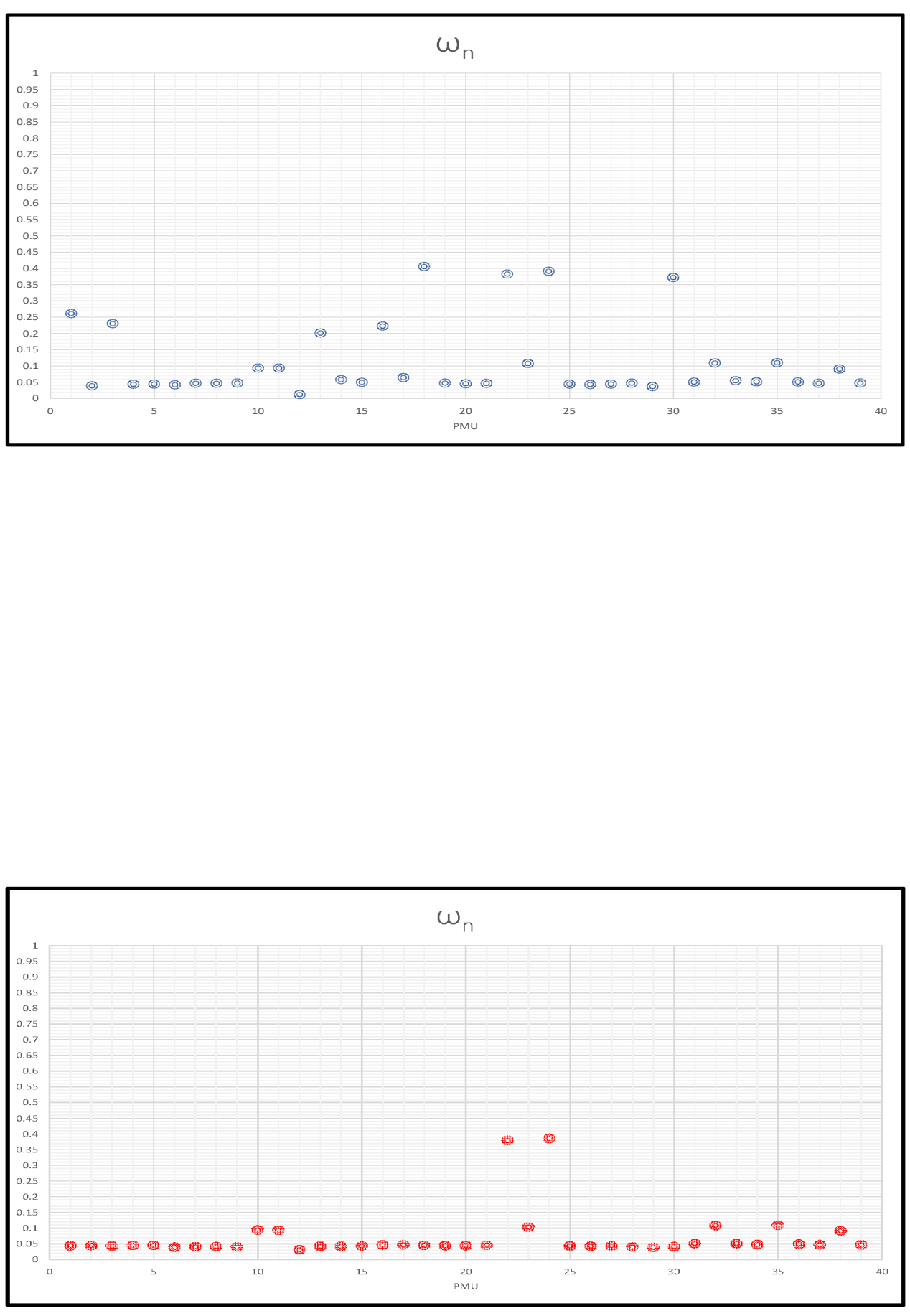

Figure A.2: All (blue) vs. Truncated (red) PMU Data-Natural Frequency $\left(\omega_{n}\right)$ 

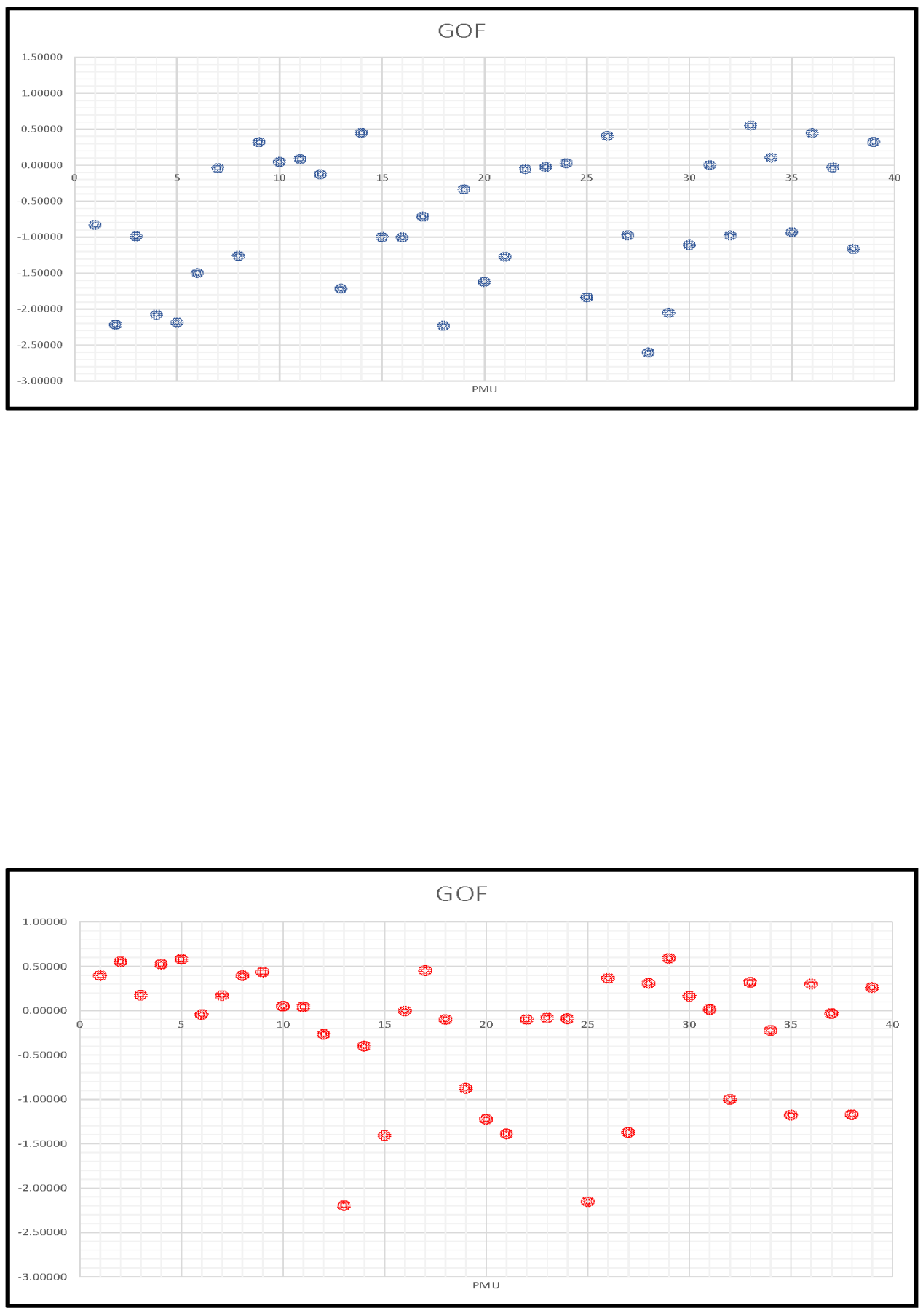

Figure A.3: All (blue) vs. Truncated (red) PMU Data-Goodness of Fit (GOF) 

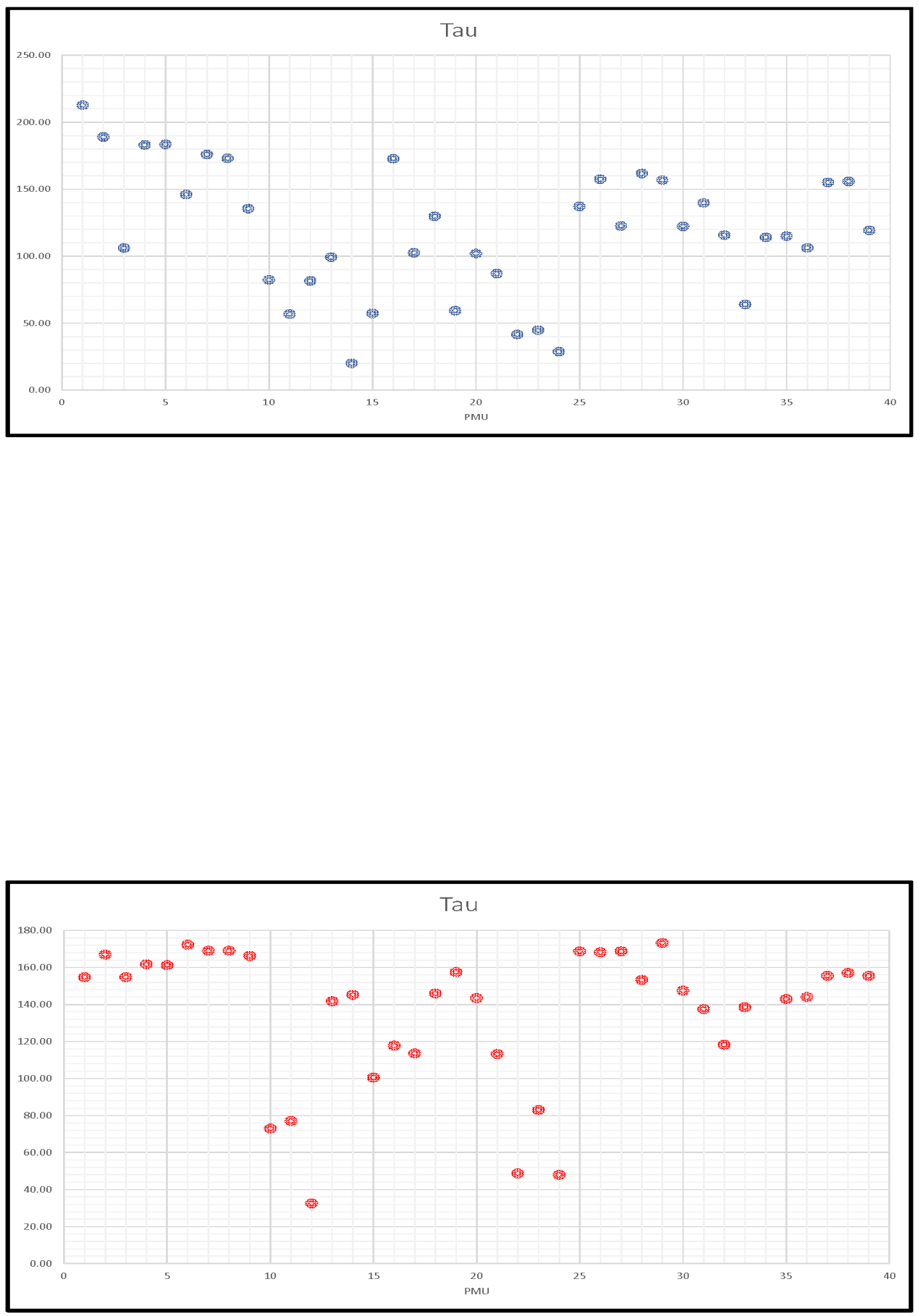

Figure A.4: All (blue) vs. Truncated (red) PMU Data-Time Constant $(\tau)$ 

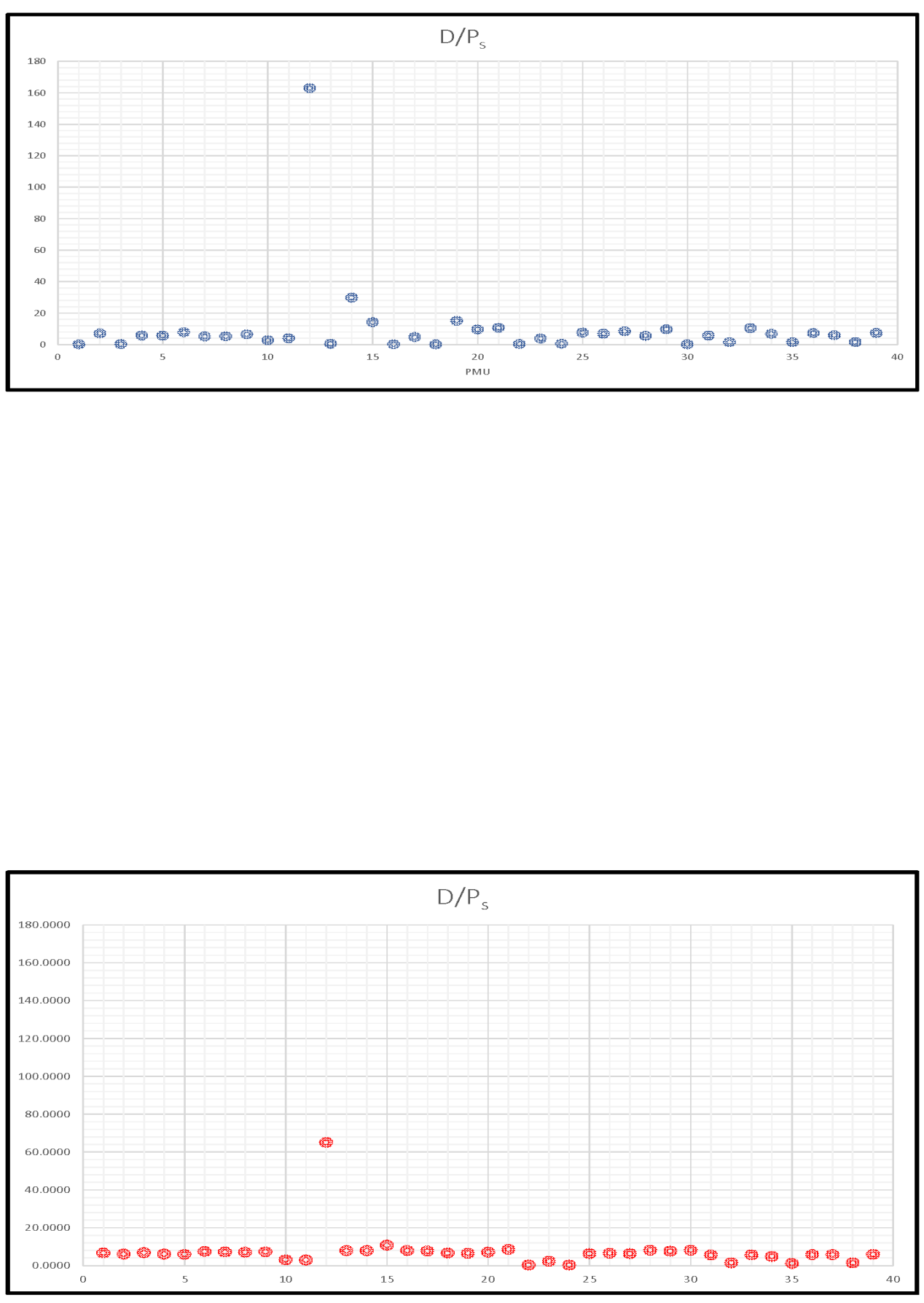

Figure A.5: All (blue) vs. Truncated (red) PMU Data-Damping Coefficient $\left(\frac{D}{P_{s}}\right)$ 

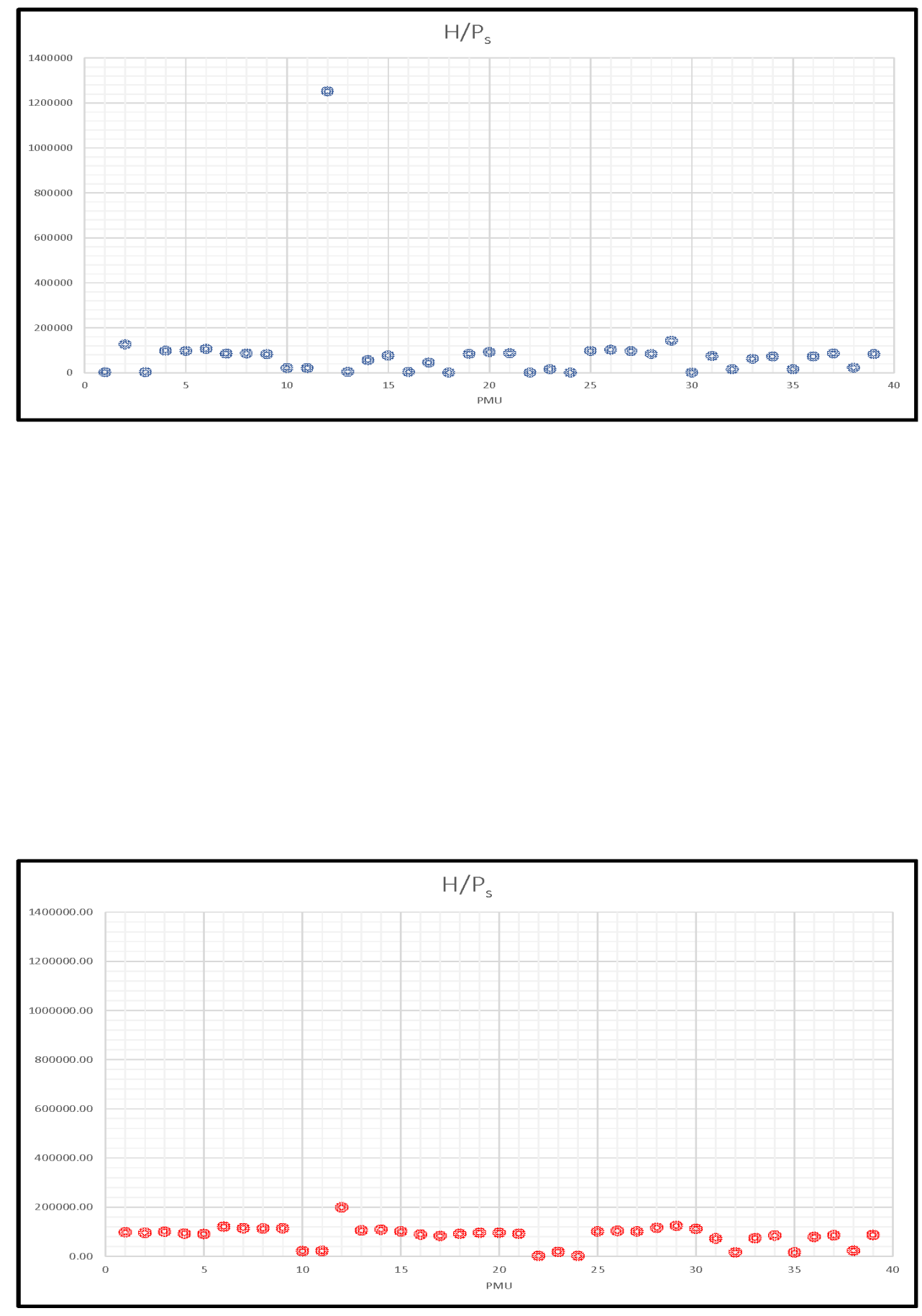

Figure A.6: All (blue) vs. Truncated (red) PMU Data-Inertia Constant $\left(\frac{H}{P_{s}}\right)$ 
Appendix B: Event Signals

B.1 Event Signals 

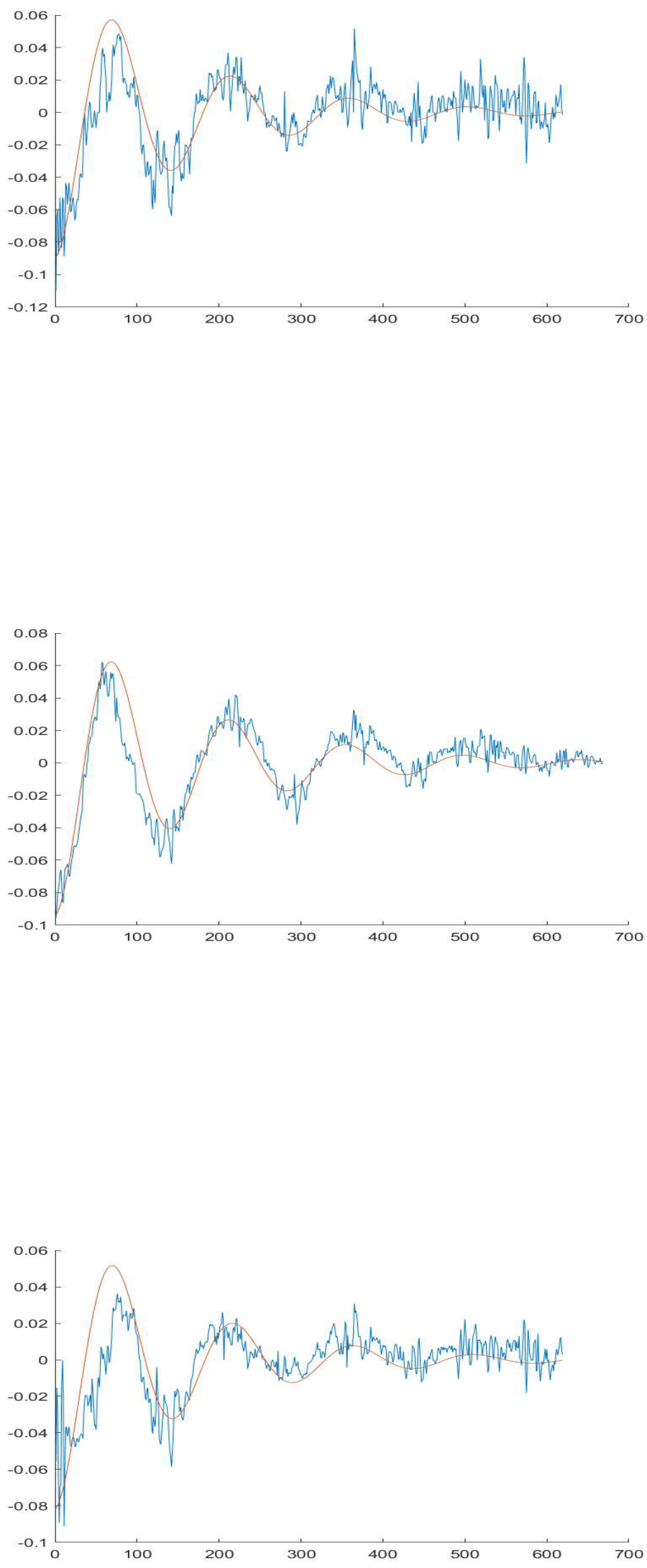

Figure B.1: Event 1: Outage on BPA DC line going from the Dalles, OR area down to California. PMU1-PMU3 listed top to bottom. 

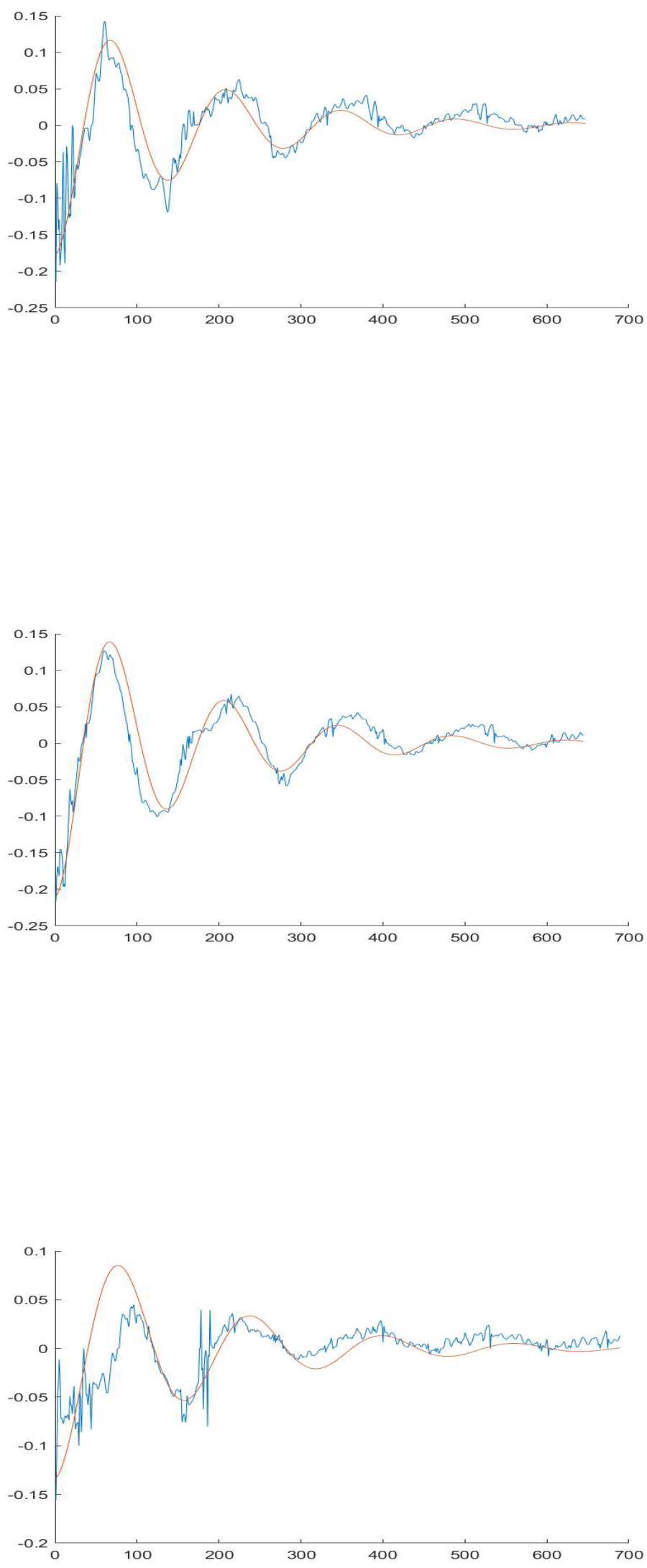

Figure B.2: Event 2: Line trip that led to a $2600 \mathrm{MW}$ gen drop. PMU1-PMU3 listed top to bottom. 

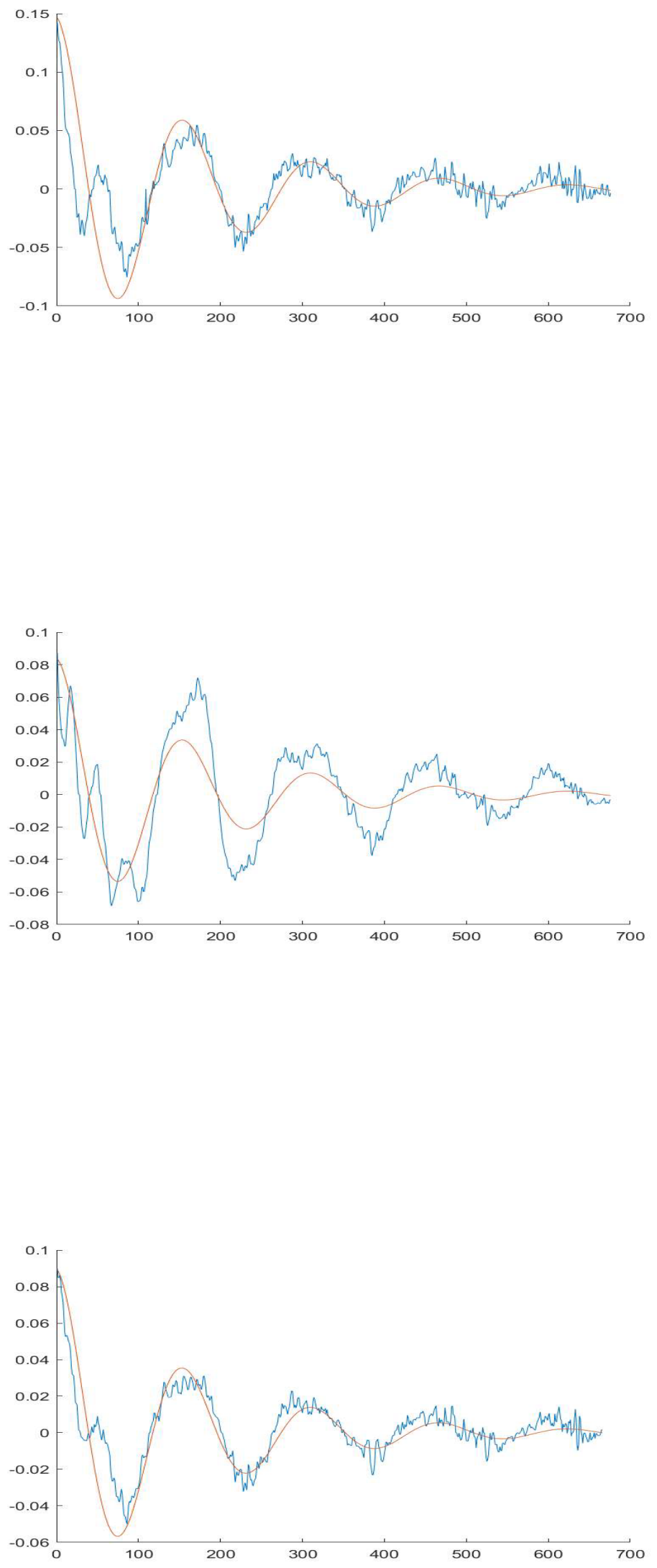

Figure B.3: Event 3: Insertion of the $1400 \mathrm{MW}$ brake resistor at Chief Joseph. PMU1-PMU3 listed top to bottom. 

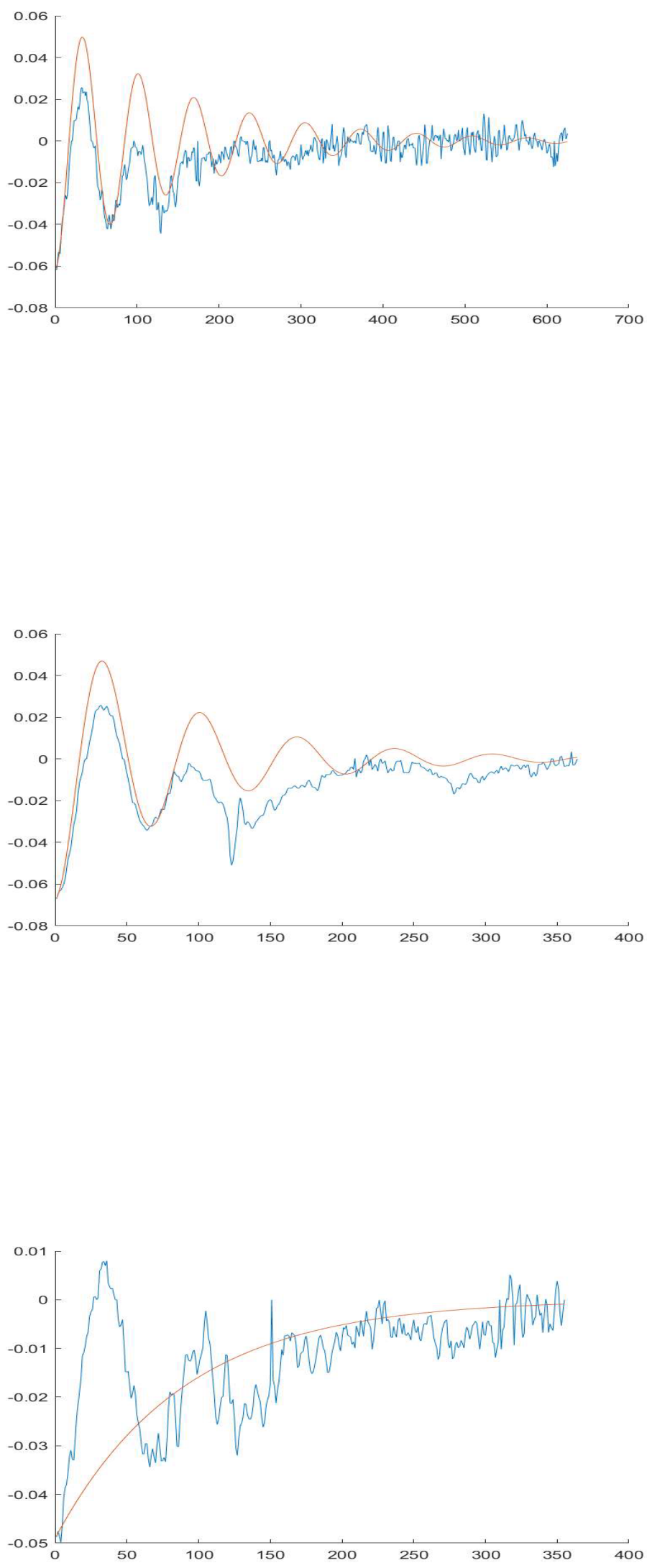

Figure B.4: Event 4: Generator outage in Montana. PMU1-PMU3 listed top to bottom. 

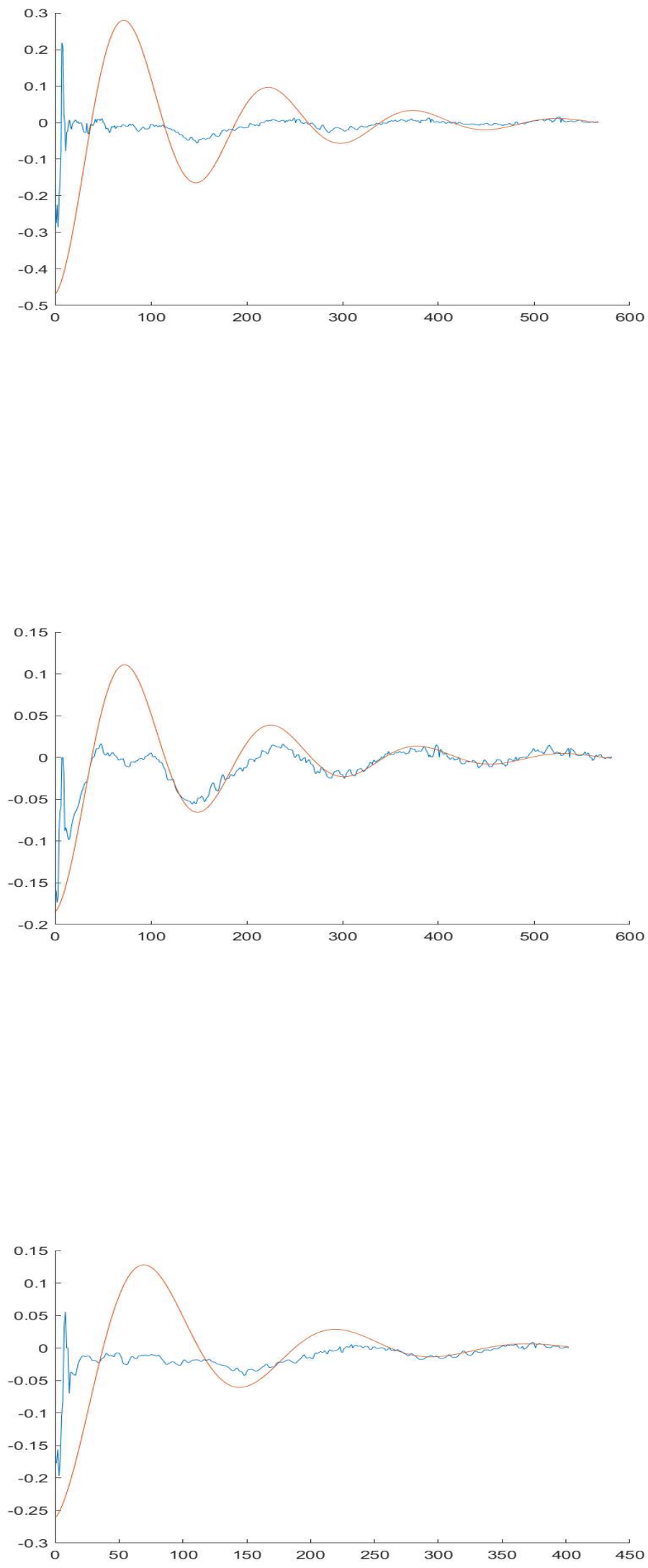

Figure B.5: Event 5: Generator outage in the Lower Columbia region. PMU1-PMU3 listed top to bottom. 

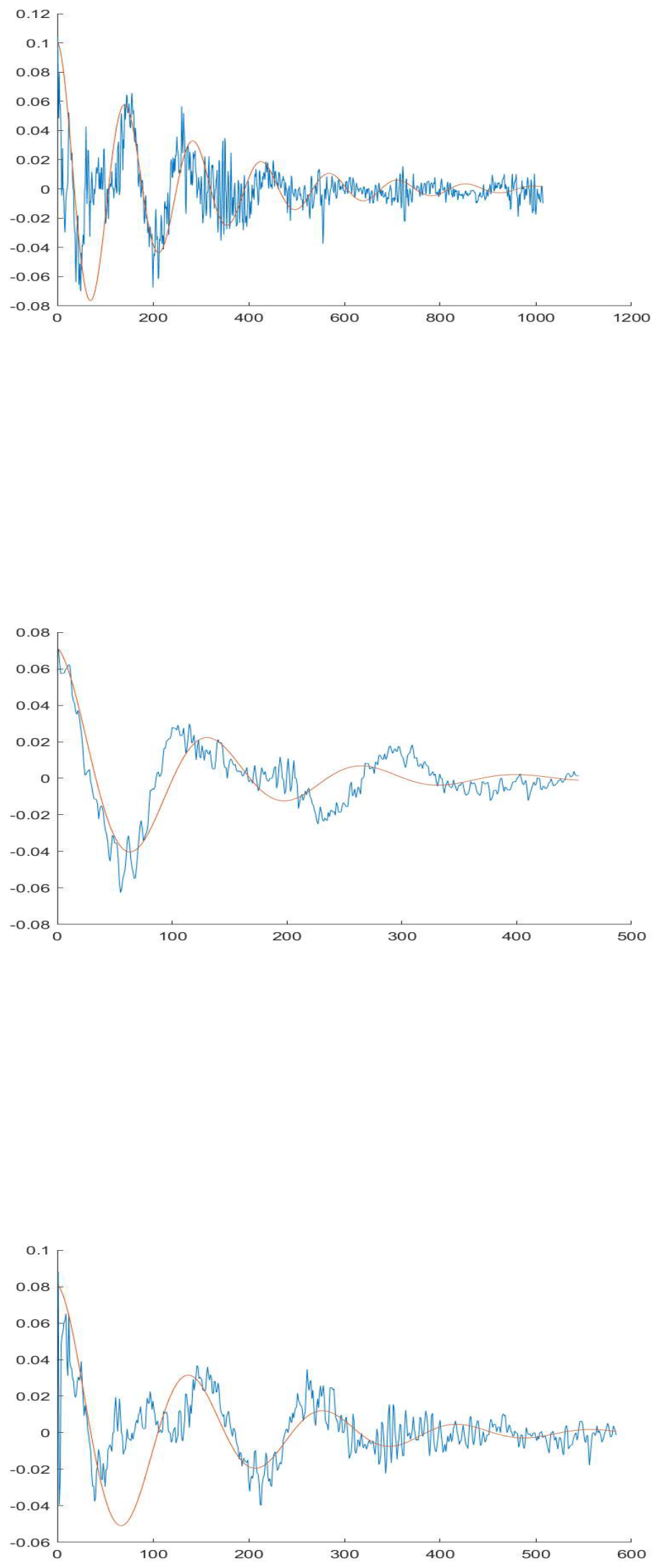

Figure B.6: Event 6: Fault on a couple of lines at one station, causing a loss of over 2200 MW. PMU1-PMU3 listed top to bottom. 

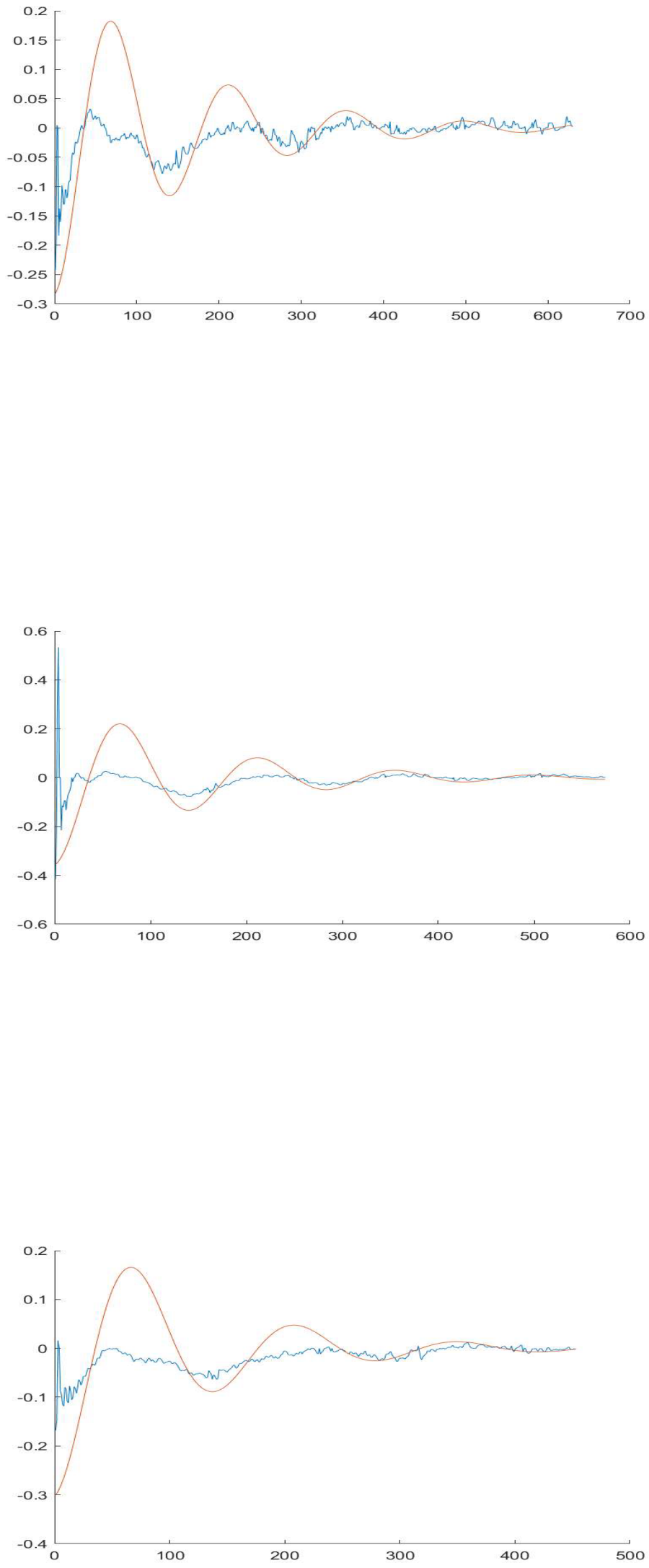

Figure B.7: Event 7: Line trip leading to a $1400 \mathrm{MW}$ generation drop. PMU1-PMU3 listed top to bottom. 

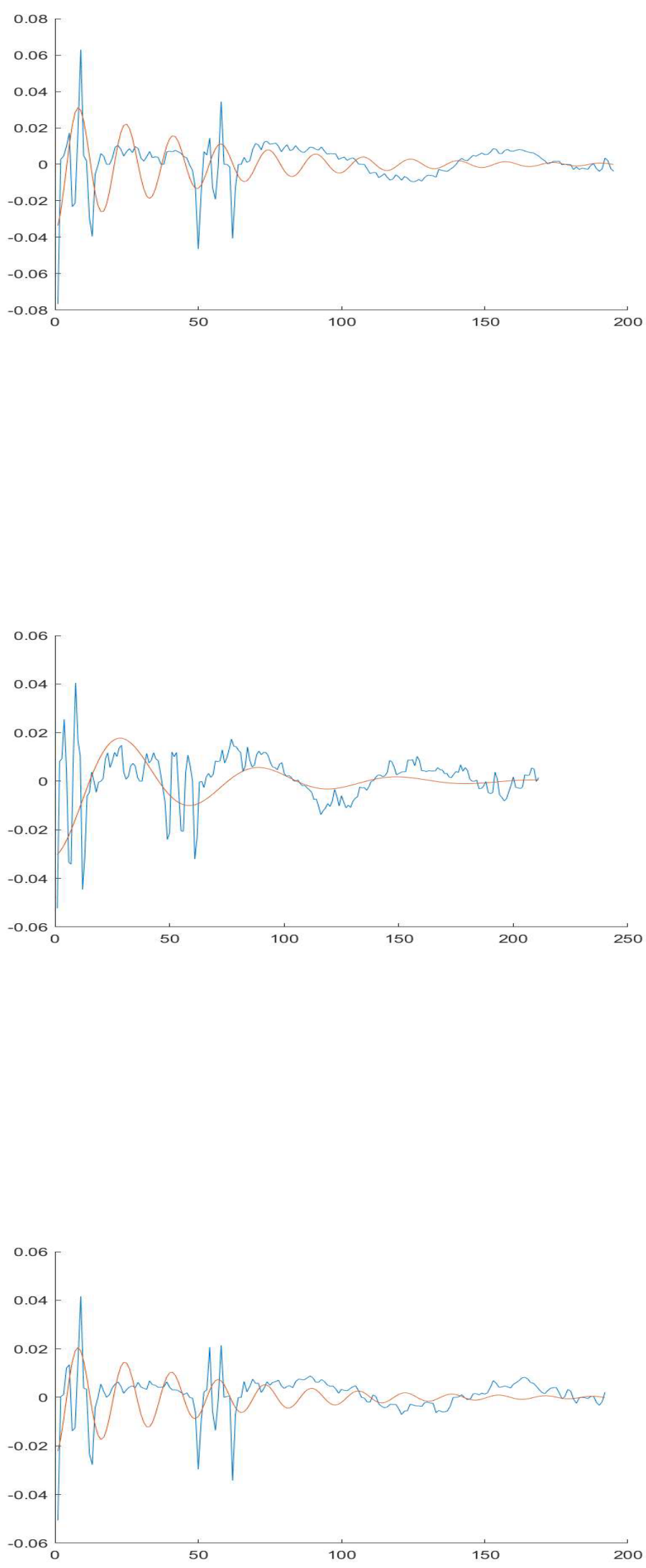

Figure B.8: Event 8: Line trip on a major line bringing generation from Montana. PMU1-PMU3 listed top to bottom. 

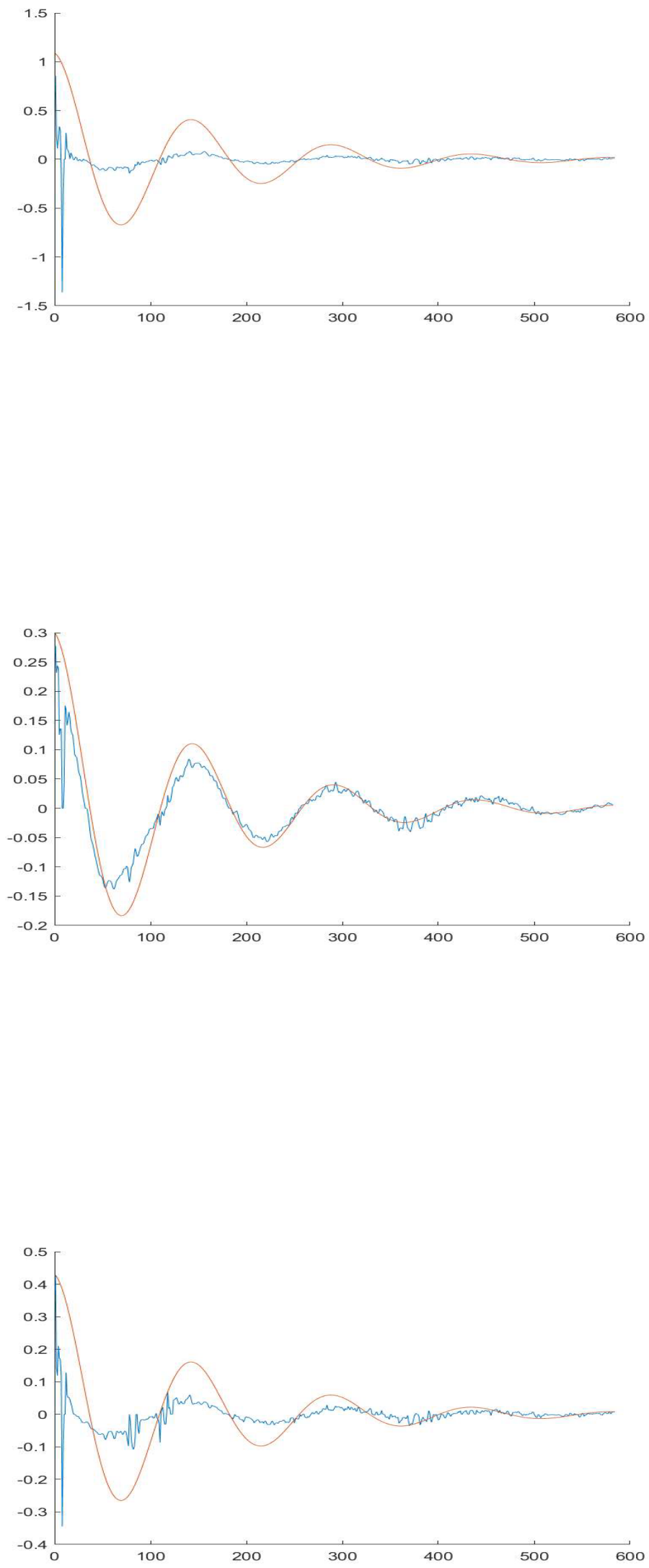

Figure B.9: Event 9: Outage on BPA DC line (similar to event 1 from the previous set). PMU1-PMU3 listed top to bottom. 

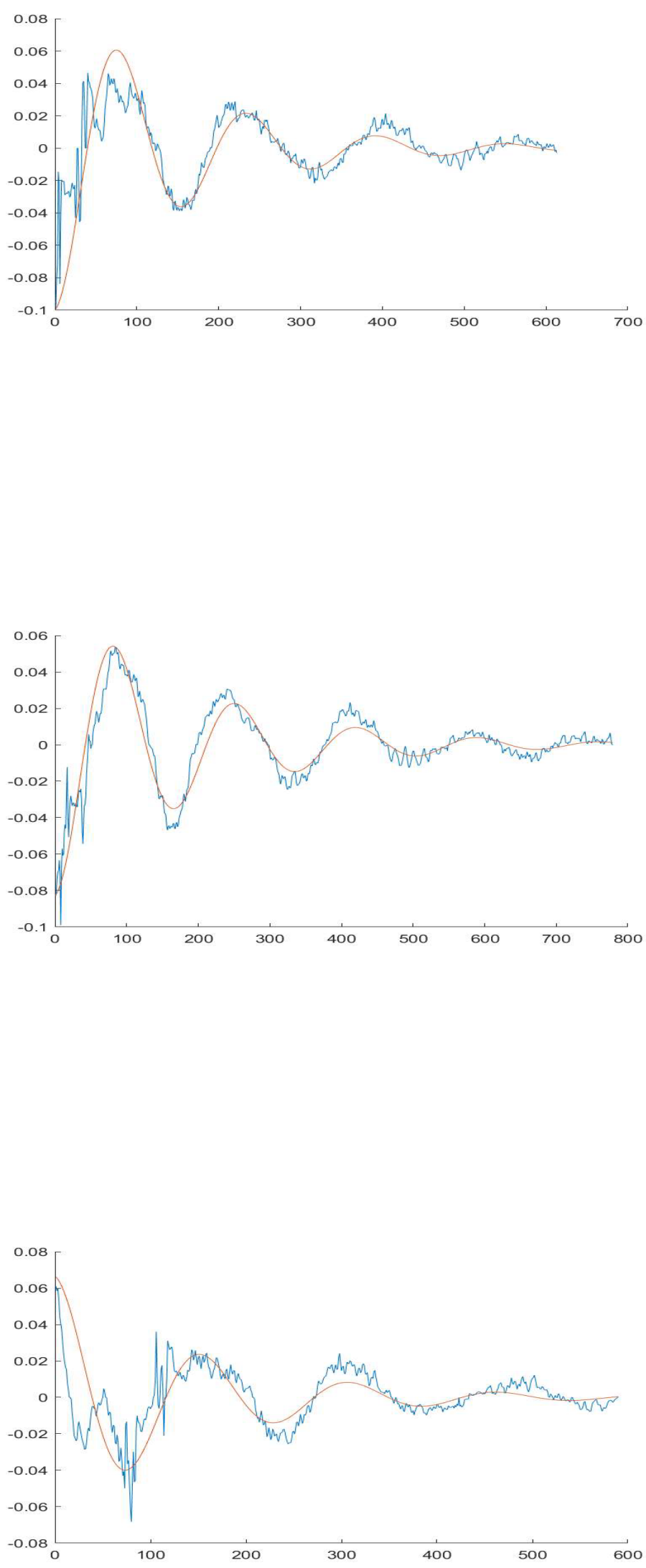

Figure B.10: Event 10: Trip/reclose/trip on a line in the lower Columbia region. PMU1-PMU3 listed top to bottom. 

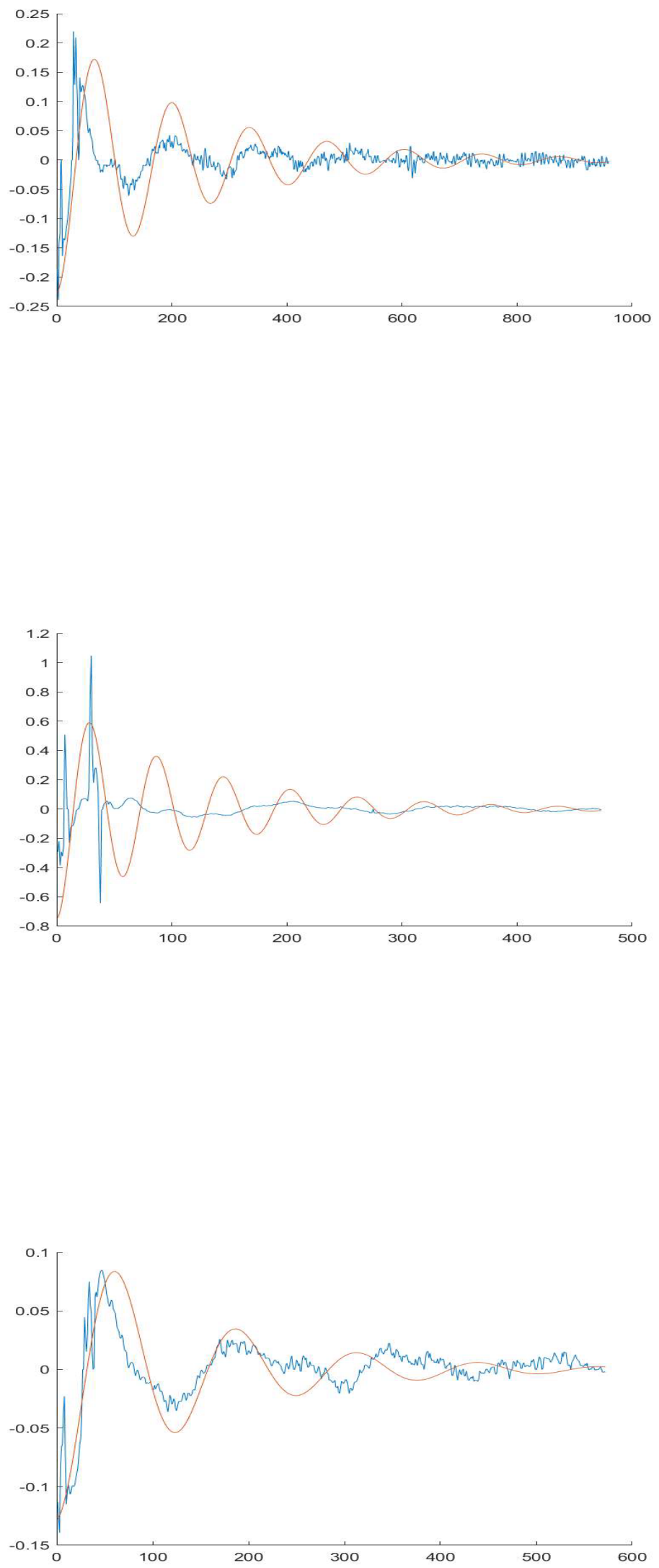

Figure B.11: Event 11: Insertion of the $1400 \mathrm{MW}$ brake resistor at Chief Joseph. PMU1-PMU3 listed top to bottom. 

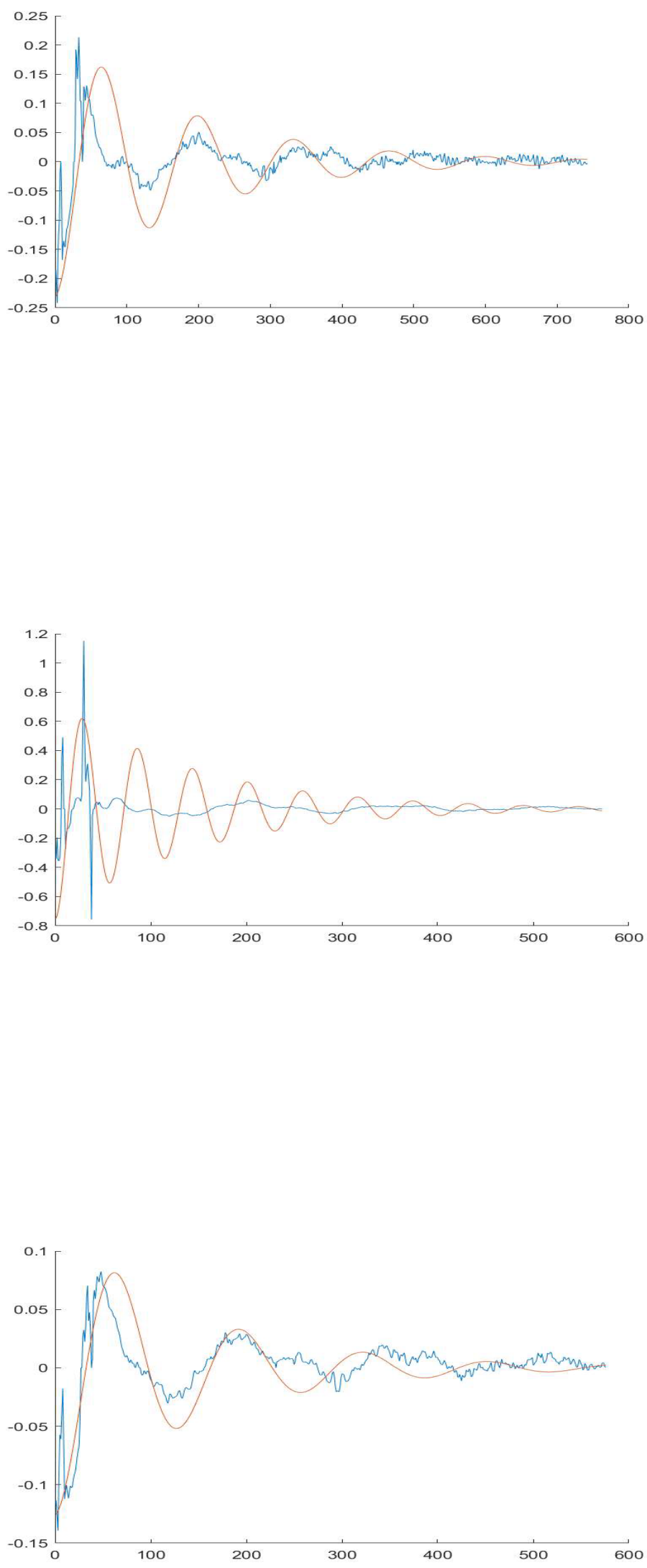

Figure B.12: Event 12: Insertion of the $1400 \mathrm{MW}$ brake resistor at Chief Joseph. PMU1-PMU3 listed top to bottom. 

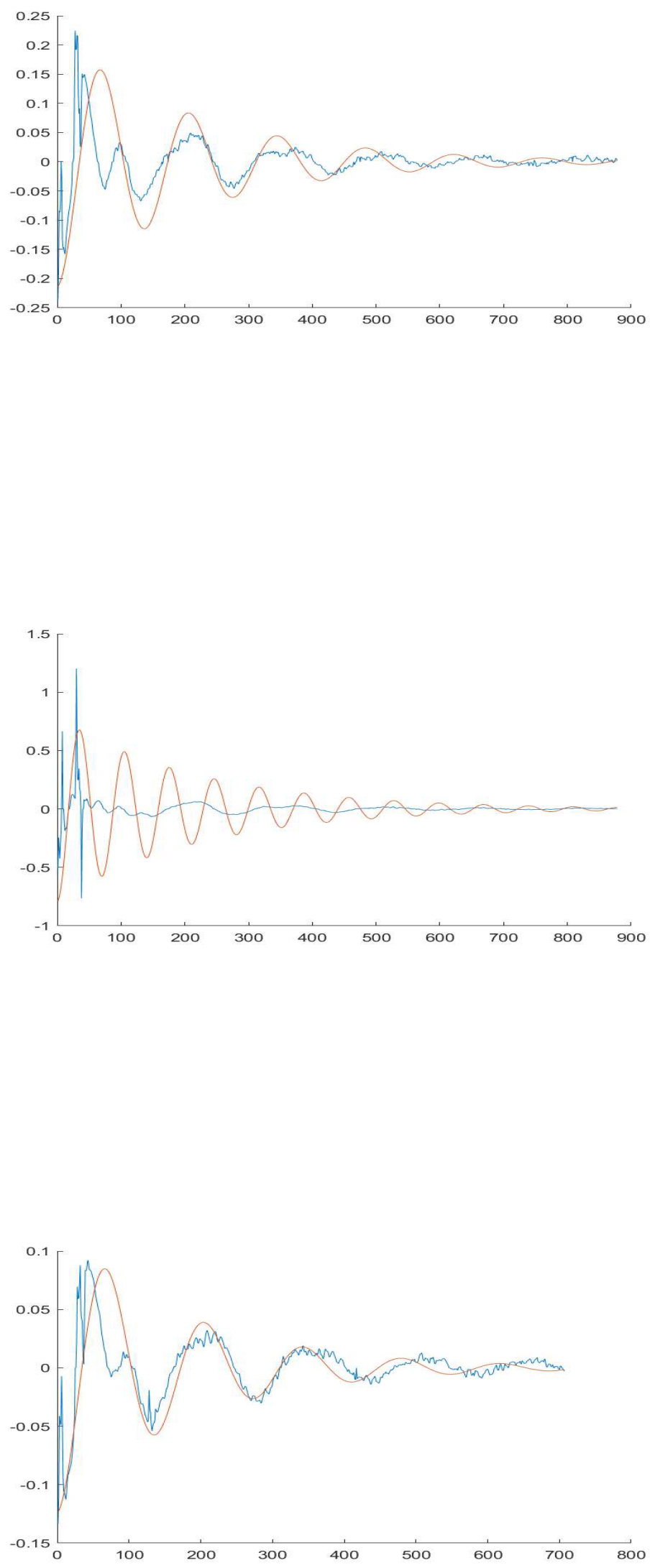

Figure B.13: Event 13: Insertion of the $1400 \mathrm{MW}$ brake resistor at Chief Joseph. PMU1-PMU3 listed top to bottom. 
Appendix C: Fit Filter Program

\section{C.1 MATLAB Code}




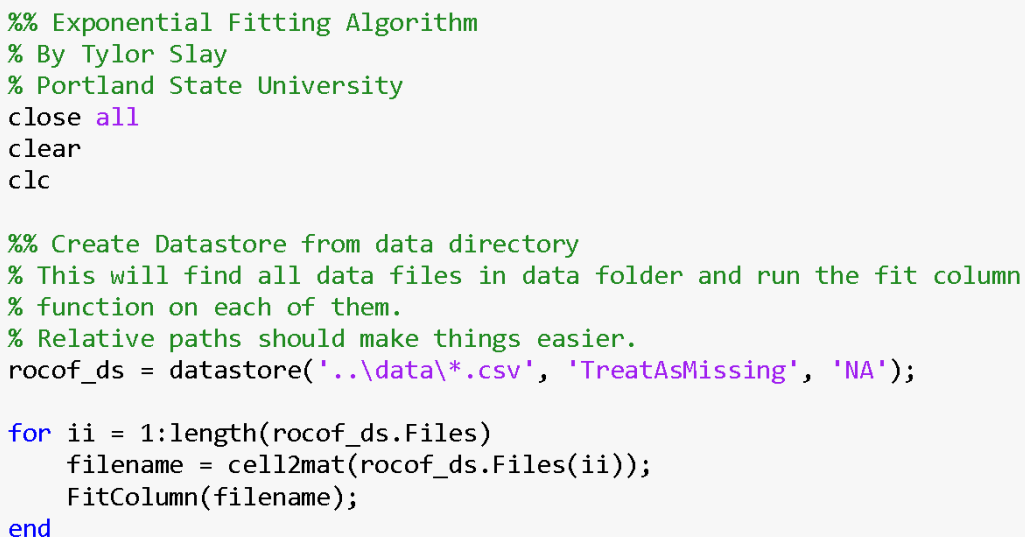

Figure C.1: MATLAB fit program code for second-order underdamped system. 


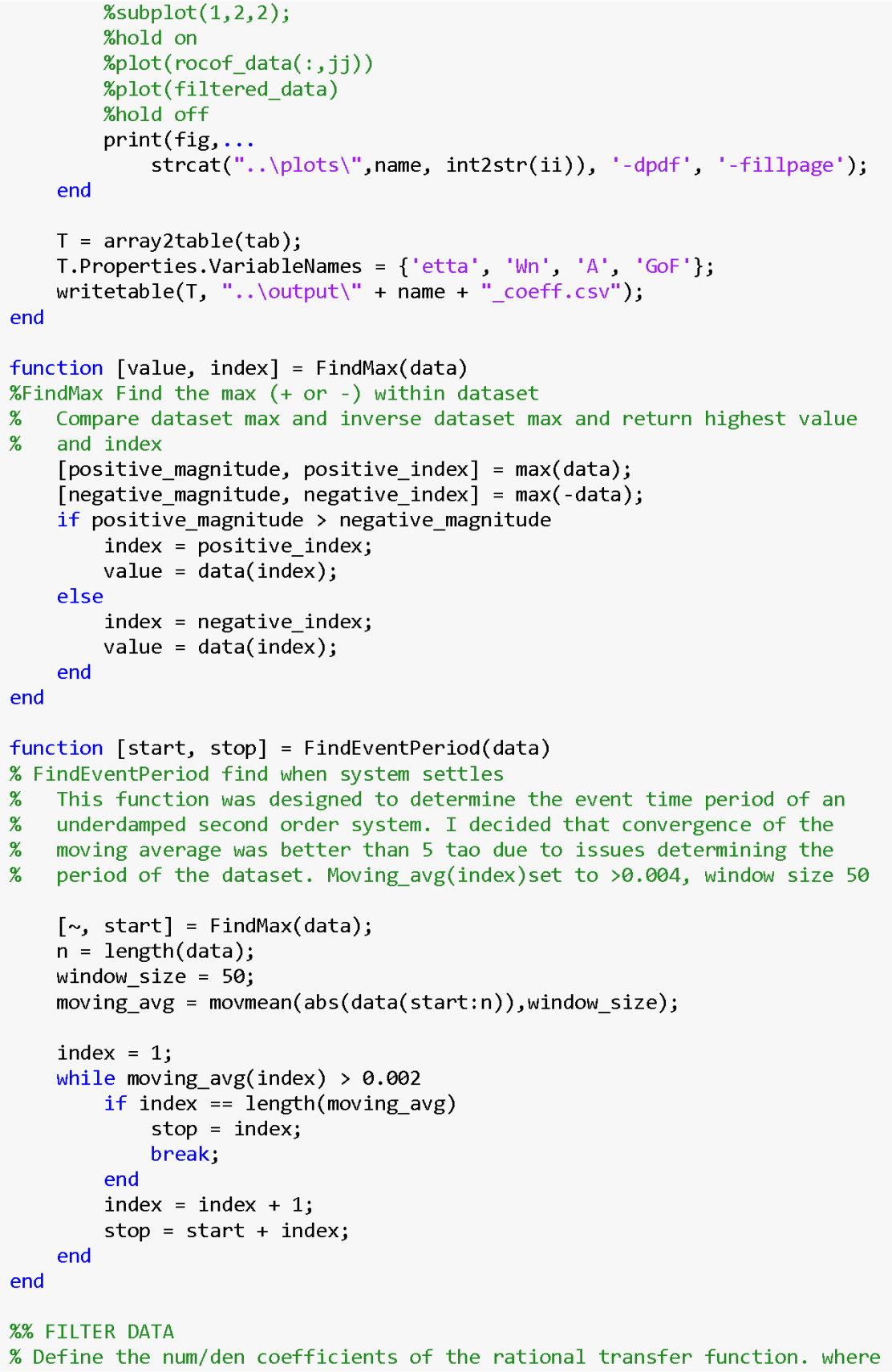

Figure C.2: MATLAB fit program code for second-order underdamped system. 


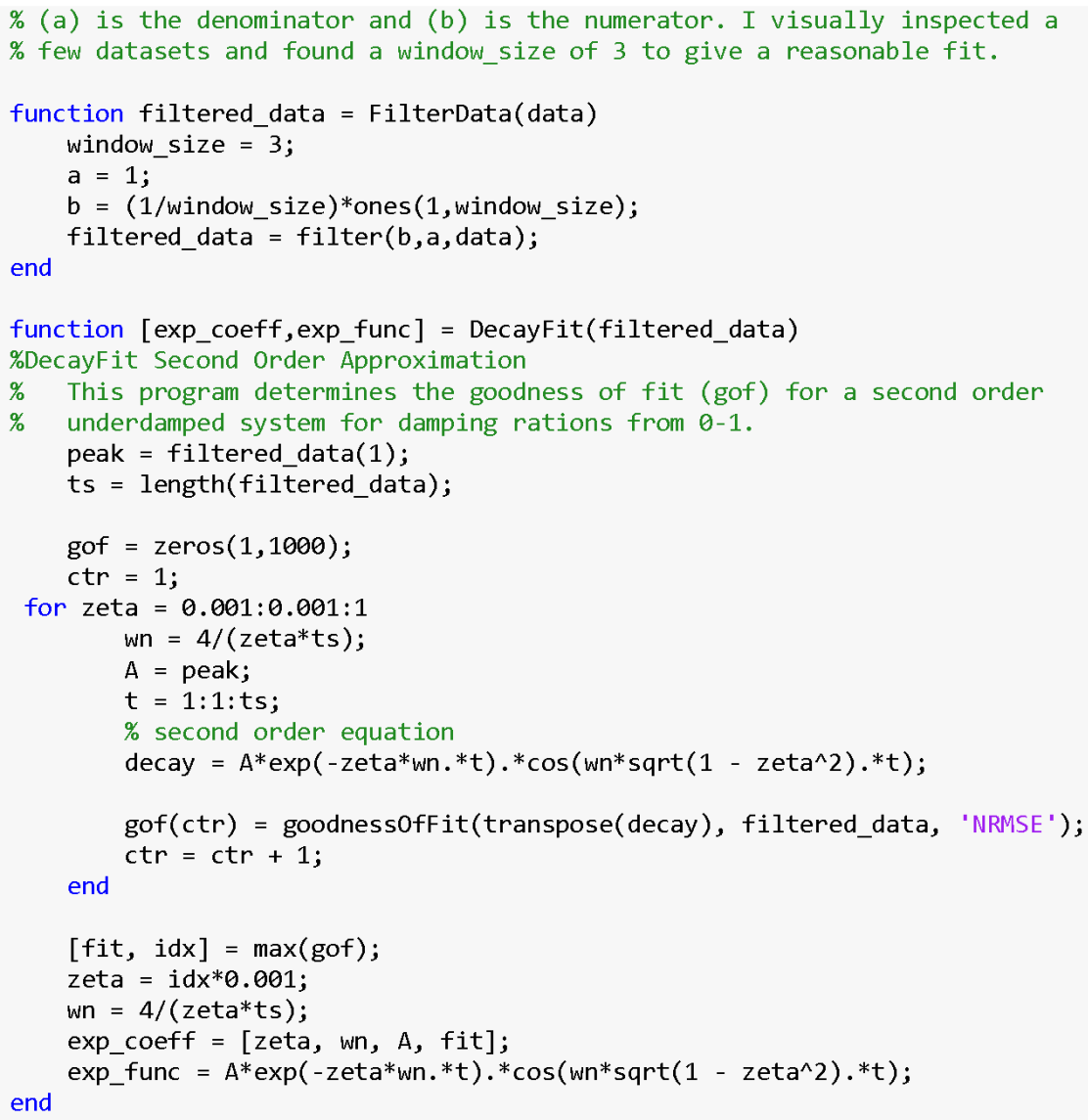

Figure C.3: MATLAB fit program code for second-order underdamped system. 